\title{
CLEAN CAST STEEL TECHNOLOGY, PHASE IV
}

Final TECHNICAL REPORT

For the Period of October 1, 2000 through september 30, 2002

\section{DE-FC07-99ID13838}

\section{INTRODUCTION}

The objective of the Clean Cast Steel Technology Program was to improve casting product quality by removing or minimizing oxide defects and to allow the production of higher integrity castings for high speed machining lines. Previous research has concentrated on macro-inclusions that break, chip, or crack machine tool cutters and drills and cause immediate shutdown of the machining lines.

Work in the past two years has been focused on optimizing pouring techniques, including metal stream shrouding and ladle design and evaluating in-mold devices such as filters and filterflow control devices for their ability to reduce macroinclusions. The program has also focused on determining the sources of heat-to-heat variations in metal cleanliness. Four foundry trials have demonstrated that modifications of the furnace practice can improve the quality of steel castings produced from 30 to $40 \%$.

The overall goal of the project is to reduce the amount of surface macro-inclusions and improve the machinability of steel castings. Macro-inclusions have been identified by industrial sponsors as a major barrier to improving the quality and marketability of steel castings.

Prior research has clearly demonstrated that the quality of castings poured from a heat can be influenced by the melting process. By slight modifications in the melting process, improvements in casting quality ranging from 30 to $50 \%$ have been confirmed on the foundry floor. Currently, the clean Cast steel Technology project is developing an engineering rationale for the effect of these process changes on casting quality. Furnace slag composition has been found to be an important factor in reducing macro-inclusions.

Several foundries have improved their casting quality by deoxidizing the slag in the furnace after blocking the heat. Foundry trials have been conducted to evaluate the effects of slag modification during melting on casting quality. The ability of a foundry to reduce the incidence of macro-inclusions through melting process modification can reduce energy usage and postcasting cost and generally adds no cost to the melting process.

Pouring operations are a major source of macro-inclusions in steel castings. Poor pouring techniques introduce atmospheric 
oxygen to the metal stream and form oxide inclusions. Techniques such as ladle shrouding, properly sizing the ladle to the mold, and reducing metal drop height were shown to significantly reduce macro-inclusion formation.

Recent research has focused on examining the effects of gating design on metal velocity and surface turbulence, which influence inclusion formation. Existing steel foundry gating practice also contains some items such as sprue wells and runner extensions which reduce yield are believed not thought to improve casting quality. Research will continue with the steel foundry industry to produce guidelines on how to properly gate steel castings to minimize macro-inclusion formation.

Most steel castings require some machining before use. Although machinability enhances the marketability and profitability of a casting, it can vary significantly depending on which steel foundry casts the part. Recent foundry experience has shown that castings identical in composition, shape, and heat treatment but cast at two different foundries had widely different machining characteristics. This demonstrates that other variables, not currently measured in the foundry, are controlling the machinability of steel. One research task has focused on developing methods to quantify the machinability of steel and understanding the metallurgical factors that affect machinability. 
CLEAN CAST STEEL TECHNOLOGY, PHASE IV

YEARLY TECHNICAL REPORT

\section{Clean/Dirty Heat Trials - Slag Modification.}

The Steel Founders' Society of America (SFSA) and The Department of Energy (DOE) have sponsored a Clean Cast Technology Program at The University of Alabama at Birmingham (UAB) for the past ten years. The objective of this program has been to improve steel casting production quality by removing or minimizing oxide defects and allowing the production of higher integrity castings. Designed experiments have been conducted throughout the program to evaluate new clean steel technologies. In two designed experiments involving bottom pour nozzle configuration and filtration, a large number of heats were poured for each trial. Heat-to-heat variation was shown to be an important factor in the overall casting quality. (1-3)

Long-term trials have been conducted in four foundries to determine if a causative relationship could be found between melting and pouring practice variables and the casting quality as measured by the number of surface oxide inclusions. Information on casting quality and metal and casting processes was collected over the length of each trial. After statistical analysis and discussions with foundry personnel, variables that significantly affected heat quality were selected. Additional heats were poured at the foundries with the selected variables at the optimum levels to verify the improvement in heat quality.

Harrison Steel Castings was the only foundry using an acid practice. Since a large amount of heat information was collected at this foundry during and after the trial, a thermo-chemical analysis was conducted.

Six variables that significantly affected heat quality. Four of the variables, including metallostatic head pressure and furnace tapping rate, were related to pouring operations and the entrainment of atmospheric oxygen. However, other important variables included metal composition after the boil and before the block. The foundry discovered that if the silicon and manganese concentrations after the boil were higher than typical, the resulting castings were cleaner. (4) Subsequent analysis showed that the $\mathrm{Mn}$ and $\mathrm{Si}$ residuals usually tracked each other, as shown in Figure 1. This suggested that only one of the elements actually controlled the reaction. The analysis also revealed a correlation between bath temperature and residual levels, as shown in Figure 2. As the bath temperature was increased, the silicon and manganese concentrations increase.

Several thermodynamic models were examined to explain the data and provide the steel foundry industry with guidelines to improve heat and casting quality. 


\subsection{Thermodynamic Analysis of the Problem}

Conceptually, cleanliness is tied to either (1) the amount of easily oxidized elements in steel or (2) the residual oxygen amount or activity in the steel which could form oxides as the steel cooled during solidification. The following material provides some definitions from metallurgical thermodynamics followed by a simplified acid slag model that accounts for the compositional effects in casting surfaces.

\subsubsection{Definitions}

This section reviews the definitions of thermodynamic terms used in the model.

Equilibrium in terms of metallurgical processes can be stated from Gibbs Free Energy, G, that a small perturbation of the system does not change the Gibbs Free Energy because the system is near a minimum value of energy, i.e.:

$\Delta G=0=\Delta G^{o}+R T \ln \frac{a_{p 1}^{v p 1} \bullet a_{p 2}^{v^{p 2}} \cdots}{a_{r 1}^{\theta_{1} 1} \bullet a_{r 2}^{v r 2} \cdots}$

where the $a^{\prime} s$ are activities and the $v^{\prime} s$ are the stoichiometric coefficients. The $\Delta G^{\circ}$ refers to reactants and products in their standard states, i.e., pure gases at one atmosphere pressure, pure elements and compound as separate phases. 


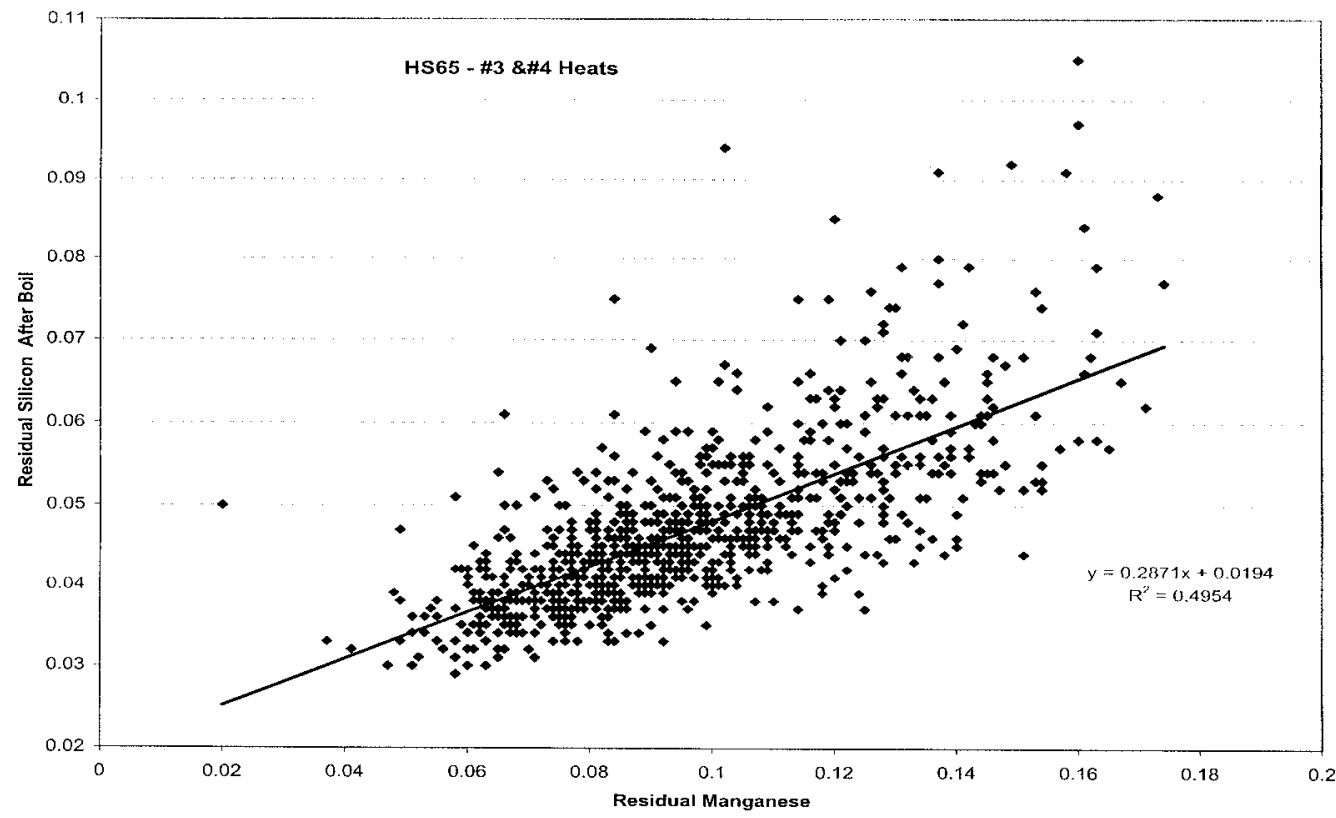

Figure 1. Correlation between residual manganese and silicon after oxygen blow. (4)

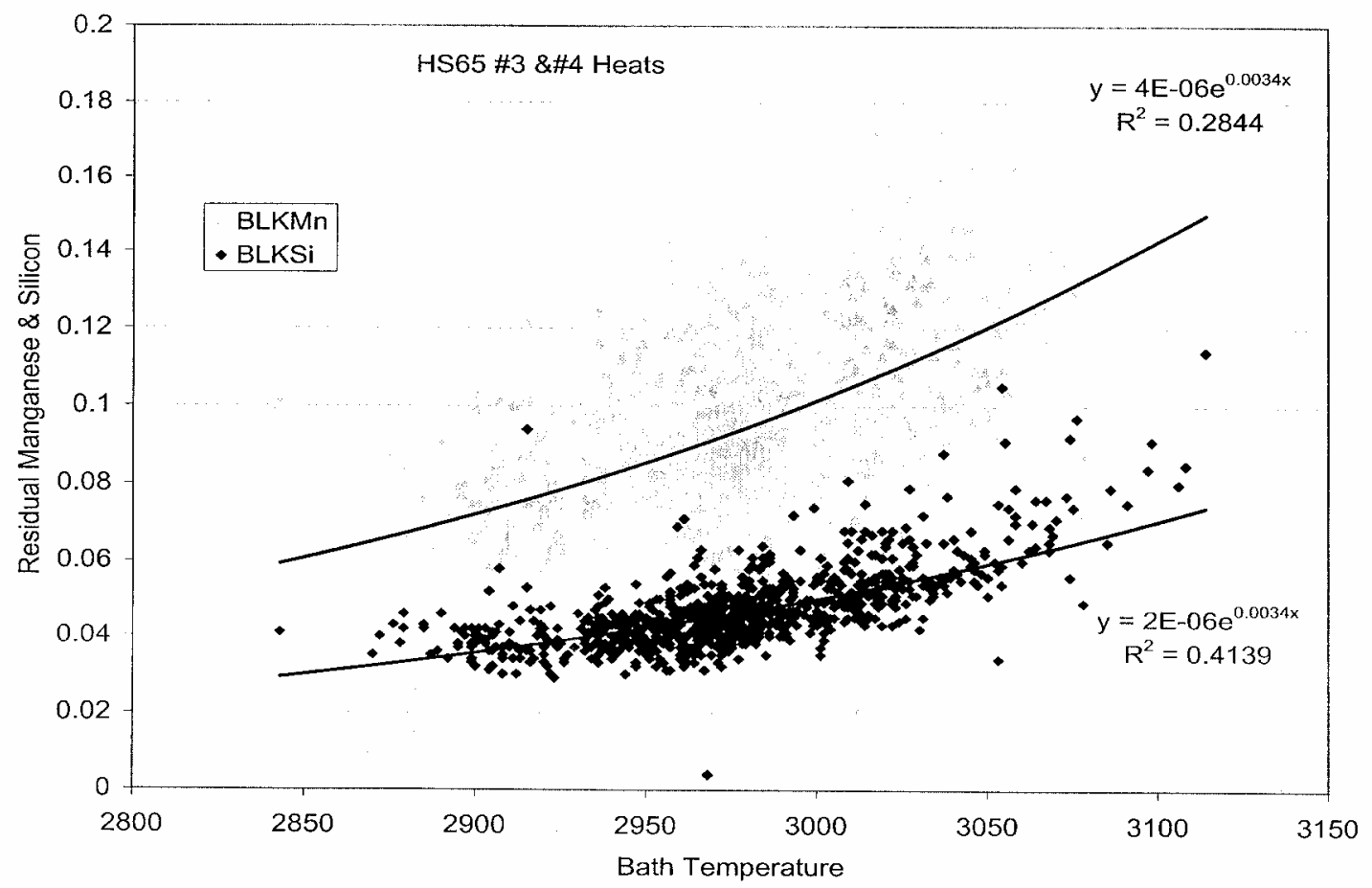

Figure 2. Correlation between bath temperature and manganese and silicon residuals. (4) 
For example, the oxidation of a metal $M$ to an oxide $M O$ is considered:

$M+1 / 2 O_{2}=M O$

then equation 1 becomes :

$$
\Delta G^{o}=-R T \ln \frac{a_{M O}}{a_{M} \bullet a_{o_{2}}^{1 / 2}}
$$

\subsection{Solution Description}

Raoult's law is used to describe activities according to the standard states mentioned above, i.e., the pure elements and compounds at the temperature and pressure of interest. Raoult's law relates activities in solution to the mole fraction, with mole fraction of one (the pure single phase element or compound) being unit activity or the standard state. Raoult's law states that (as an approximation) activity in solution is proportional to mole fraction:

$a=\gamma \bullet X$

where a is activity, $x$ is mole fraction in solution, and $\gamma$ is activity coefficient.

Henrian activity, $h$, is related to weight percent of the elements being considered. Henrian activity extrapolates extremely dilute solution behavior to practical composition ranges.

$h=f \bullet(w t . \%)$

The standard state is obtained by extrapolating the slope of the activity curve in a dilute solution to a concentration of one weight percent. The real activity is obtained by multiplying the percent concentration by an activity coefficient, $f$. If the standard state is changed, free energy change of reaction changes and modifications must be made to take the changes in standard state into account (5).

The activity of the element in solution depends on the amount of that element in the solution and on the amounts of other elements in solution. The element of interest may have an attractive or repulsive interaction with the other elements in solution. This non-linearity is represented by the activity coefficient curve using a Taylor expansion and limiting the 
approximation to the first order terms. This is also called the "Universal Coefficient" method or the Wagner method. Therefore, for the Raoult's law model (pure element as the standard state) the equation becomes:

$\ln \gamma_{j}=\ln \gamma_{j}^{o}+\sum_{i} x_{i} \cdot \varepsilon_{j}^{i}$

$\gamma_{j}$ is the activity coefficient of element $j$, and the summation includes the case where $i=j$.

$\boldsymbol{E}^{i}{ }_{j}$ is the interaction coefficient of element $i$ on $j$.

If the Henrian activity (percent scale) is used:

$\log _{10} f_{j}=\sum_{i}(\% i) \bullet\left(e_{j}^{i}\right)$

again including the case where $i=j$.

It was initially thought that equilibrium with a highly oxidizable element in the steel solution could be found that would be the controlling reaction. That assumed an equilibrium could be found that involved oxygen from a gaseous or solute form, and an oxide reaction product at a known activity. Compositions during tapping and pouring were examined, but a good correlation was not found.

\subsubsection{Simplified Acid Model}

It was known that residuals in higher boil temperature, higher silicon and manganese in the furnace produced cleaner castings.(4) This was counter to the initial hypothesis because the controlling equilibria should give higher oxygen in solution in the melt at the higher temperatures. (6)

The acid melt was then modeled with a simplified ironcarbon-silicon-manganese alloy. The reaction product should be a mixed oxide solution or a multiphase slag including one or more solutions. Fortunately, Gaskell and Rao have conducted analyses and experimental work on the manganese-oxide-silica system. (7) The activity of $\mathrm{SiO}_{2}$ in the slag varies strongly and non-linearly with the MnO content of the slag solution as illustrated in Figure 3. (7)

The activity of MnO was then calculated from the GaskellRao model and then use the equilibrium $2 \mathrm{MnO}+\mathrm{SiO}_{2}=\mathrm{Mn}_{2} \mathrm{SiO}_{4}$ was used to determine the equilibrium activity of $\mathrm{SiO}_{2}$. Working from the equation for mole fraction of $\mathrm{SiO}_{2}$ as a function of $\mathrm{MnO}$ 
activity which was shown by Gaskell and Rao, we developed a quadratic solution was developed for the activity of $\mathrm{MnO}$ :

$\mathbf{a}_{\mathrm{MnO}}=\frac{-\mathbf{b} \pm \sqrt{\mathbf{b}^{2}-4 \mathbf{a c}}}{2 \mathbf{a}}$

where :

$\mathbf{a}=-\left[\left(1 / \mathbf{N}_{\mathrm{SiO} 2}\right)-2\right] \bullet\left(\mathbf{K}_{11}-3\right)$

$\mathbf{b}=-\left(1 / \mathbf{N}_{\mathrm{SiO} 2}-2\right) \cdot\left(3-2 \mathbf{K}_{11}\right)$

$\mathbf{c}=-\left(1 / \mathbf{N}_{\mathrm{SiO} 2}-2\right) \mathbf{K}_{11}-2 \mathbf{K}_{11}$

with $\mathrm{N}_{\mathrm{SiO} 2}$ being mole fraction of silica in the slag and $\mathrm{K}_{11}$ being the equilibrium constant for the anionic polymerization reaction.

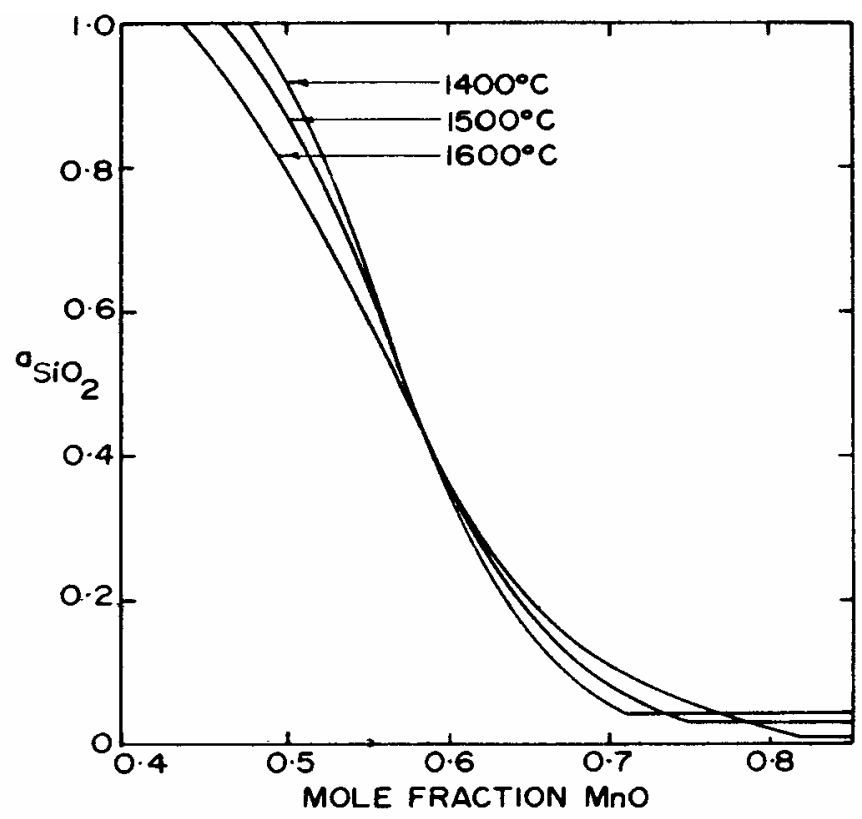

Figure 3. Activity of $\mathrm{SiO}_{2}$ as a function of MnO concentration. (7) 
The activity of $\mathrm{SiO}_{2}$ was then evaluated from the equilibrium constant for manganese orthosilicate formation using the free energies of formation shown by Gaskell and Rao.

$$
\mathbf{a}_{\mathrm{SiO}_{2}}=\frac{1}{\mathbf{K}_{\mathrm{Mn}_{2} \mathrm{SiO}_{4}} \cdot \mathbf{a}_{\mathrm{MO}}^{2}}
$$

Using the data from Pehlke (8), we can then calculate the Henrian activity of oxygen in equilibrium with the silica in solution and the silica in the slag was then calculated:

$\mathbf{h}_{\underline{0}}=\left[\frac{36700 \times \mathbf{h}_{\underline{\mathbf{S i}}}}{\mathbf{a}_{\mathbf{S i O}} 2}\right]^{-0.5}$

In general, a very high activity of $\mathrm{Mn}$ in the metal, low MnO activity in the slag, and a very low si activity in the metal are required for $\mathrm{Mn}$ to be the controlling deoxidizer. However, MnO in the slag does enhance the effectiveness of the silicon deoxidization by lowering the $\mathrm{SiO}_{2}$ activity in the slag. Manganese in solution slightly increases the activity of silicon $\left(e^{\mathrm{Mn}} \mathrm{Si}=+0.5\right)$

These calculations provide activities of the reaction products, i.e., $\mathrm{SiO}_{2}, \mathrm{MnO}$. If $\mathrm{P}_{\mathrm{Co}}=1$ atmosphere, the possible deoxidation reactions are as follows:

$\underline{\mathbf{S i} \%}+2 \underline{\mathbf{O} \%}=\mathbf{S i O}_{2}$

$\underline{\mathrm{Mn} \%}+\underline{\mathbf{O} \%}=\mathbf{M n O}$

$\underline{\mathrm{C}} \%+\underline{\mathbf{O} \%}=\mathbf{C} \mathbf{O}_{g}$

The equilibrium oxygen content in percent was calculated for each reaction over a range of compositions and temperatures, then a logic function was used to select the lowest values of oxygen content (the governing reaction result). The conditions considered in developing the solubility curves are listed in Table I. 


\begin{tabular}{|c|c|c|c|}
\hline Temperature & $\begin{array}{c}\text { Carbon } \\
\text { percent }\end{array}$ & $\begin{array}{c}\text { Percent } \\
\text { Silicon }\end{array}$ & $\begin{array}{c}\text { Percent } \\
\text { Manganese }\end{array}$ \\
\hline $1520 \mathrm{C}$ & $0.05-0.4$ & $0-0.3$ & $0-0.3$ \\
\hline $1600 \mathrm{C}$ & $0.05-0.4$ & $0-0.3$ & $0-0.3$ \\
\hline $1650 \mathrm{C}$ & $0.05-0.4$ & $0-0.3$ & $0-0.3$ \\
\hline $1700 \mathrm{C}$ & $0.05-0.4$ & $0-0.3$ & $0-0.3$ \\
\hline
\end{tabular}

Table I. Conditions and ranges used to develop solubility curves for idealized alloys.

Some of the results of applying this approach to the model carbon-silicon-manganese-iron melt are shown in Figures 4 through 7. The manganese concentration was held constant at $0.075 \%$ while curves were calculated for silicon concentrations of $0,0.05$, 0.1 , and $0.15 \%$ for each temperature. At temperatures of 1700 and $1650^{\circ} \mathrm{C}, \mathrm{SiO}_{2}$ formation may govern equilibrium oxygen content, but only at carbon content of about 0.15 or less as seen in Figures 4 and 5. As carbon content increases, the formation of carbon monoxide governs the equilibrium oxygen concentration. As temperature decreases, as illustrated in Figure 6 and 7, the $\mathrm{SiO}_{2}$ equilibrium begins to dominate over a larger carbon content range to include higher carbon concentrations. Above the control carbon concentrations, at a given temperature, the co equilibrium controls the oxygen concentration.

At lower temperatures, the $\mathrm{SiO}_{2}$ equilibrium dominates oxygen control over the entire carbon range. If the plots of oxygen concentration are examined at lower temperatures (i.e., $1520^{\circ} \mathrm{C}$ or $\left.2770^{\circ} \mathrm{F}\right)$ (Figure 7 ), it is found that the reaction:

\section{$\underline{\mathrm{Si} \%}+2 \underline{\mathbf{O} \%}=\mathrm{SiO}_{2}\left(\mathrm{in} \mathrm{SiO}_{2}-\mathrm{MnO} \_\right.$slag $)$}

controls the oxygen concentration of the metal, but higher manganese concentrations increases the MnO in the slag, thus decreasing the $\mathrm{SiO}_{2}$ activity in the slag and driving the equilibrium to lower oxygen concentrations. That is because

\section{$\frac{\mathbf{a}_{\mathrm{SiO}_{2}}}{\mathbf{h}_{\mathbf{o}}^{2} \bullet \mathbf{h}_{\mathrm{si}}}=\mathbf{K}_{\mathrm{eq}}$}

is an equilibrium quotient which varies with temperature. However, at a constant temperature and total pressure, is a fixed value. Thus, if the silica activity $\left(a_{\text {sion }}\right)$ is lowered at a fixed temperature and total pressure, the oxygen activity $\left(h_{0}\right)$ must also decrease if the activity of silicon in the melt $\left(h_{s i}\right)$ is the same. 
The reason for the shift in oxygen concentration control from the $\mathrm{CO}$ formation reaction to the $\mathrm{SiO}_{2}$ formation reaction is because the $\mathrm{CO}$ equilibrium is weakly dependent on temperature but the formation of $\mathrm{SiO}_{2}$ is more strongly temperature dependent. (9)

The calculation method for these solubility curves is summarized in Figure 8.

\subsection{Application to the case where slag composition is known}

The same calculation model has been applied to acid slags where the composition of the slag was known. The initial result, using tap temperature and after block chemistry for the analysis, showed that while the oxygen activity determined from the Si/Sio2 equilibrium was near that determined by the C/CO equilibrium, the C/CO equilibrium always gave a slightly lower oxygen activity values. If the $\mathrm{P}_{\mathrm{CO}}$ was raised to 1.5 atmospheres, there was some crossover as observed in the solubility plots in the previous section. This may be artificial, but it certainly indicates how sensitive this model is to errors in the data.

The scatter plots and trend lines were then used to determine which oxygen activity correlated with steel cleanliness. The plot of the oxygen activity versus dirt inches is shown in Figure 9 for control by the C/CO equilibrium. Similar data where control is mixed (i.e., with crossover) is presented in Figure 10. The result of that is that the C/CO limit and the mixed limit did not correlate as well with cleanliness as the oxygen activity determined by the $\mathrm{Si} / \mathrm{SiO}_{2}$ equilibrium, which is shown in Figure 11.

As pointed out by Carpenter et al, higher meltdown and endof-blow temperature produce higher silicon concentrations and less dirt. This probably reflects the stronger temperature dependence of the $\mathrm{Si} / \mathrm{SiO}_{2}$ equilibrium compared to the $\mathrm{C} / \mathrm{CO}$ equilibrium. A high residual silicon buffers the system to lower oxygen concentrations as the melt cools during tapping and pouring.

Slag/metal chemistry modeling software has been used to determine if there was a thermo-chemical rationale for the relationships between heat quality, metal chemistry, and slag chemistry. An acid slag model has been developed from previous data and the following is a description of the thermodynamics of a basic slag model. With baseline data from a basic foundry, an effort was made to determine if the acid slag model would work for a basic slag.

The composition of a variety of ladle and furnace slags were determined, and with some adjustments to the model, reasonably good predictions were made. 


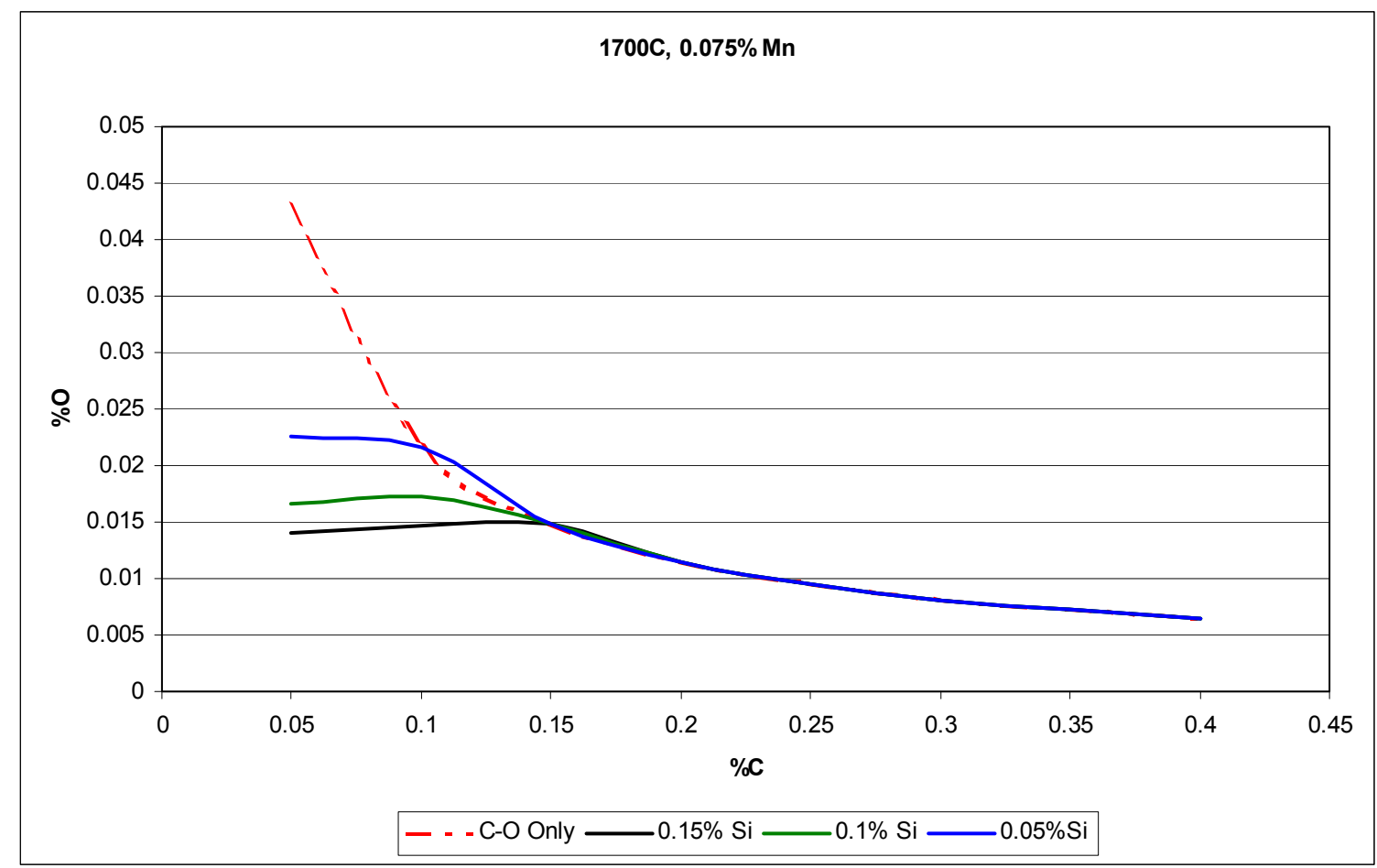

Figure 4. Concentration of oxygen as a function of carbon and silicon level - 1700C, $0.075 \% \mathrm{Mn}$.

$1650 \mathrm{C}, 0.075 \% \mathrm{Mn}$

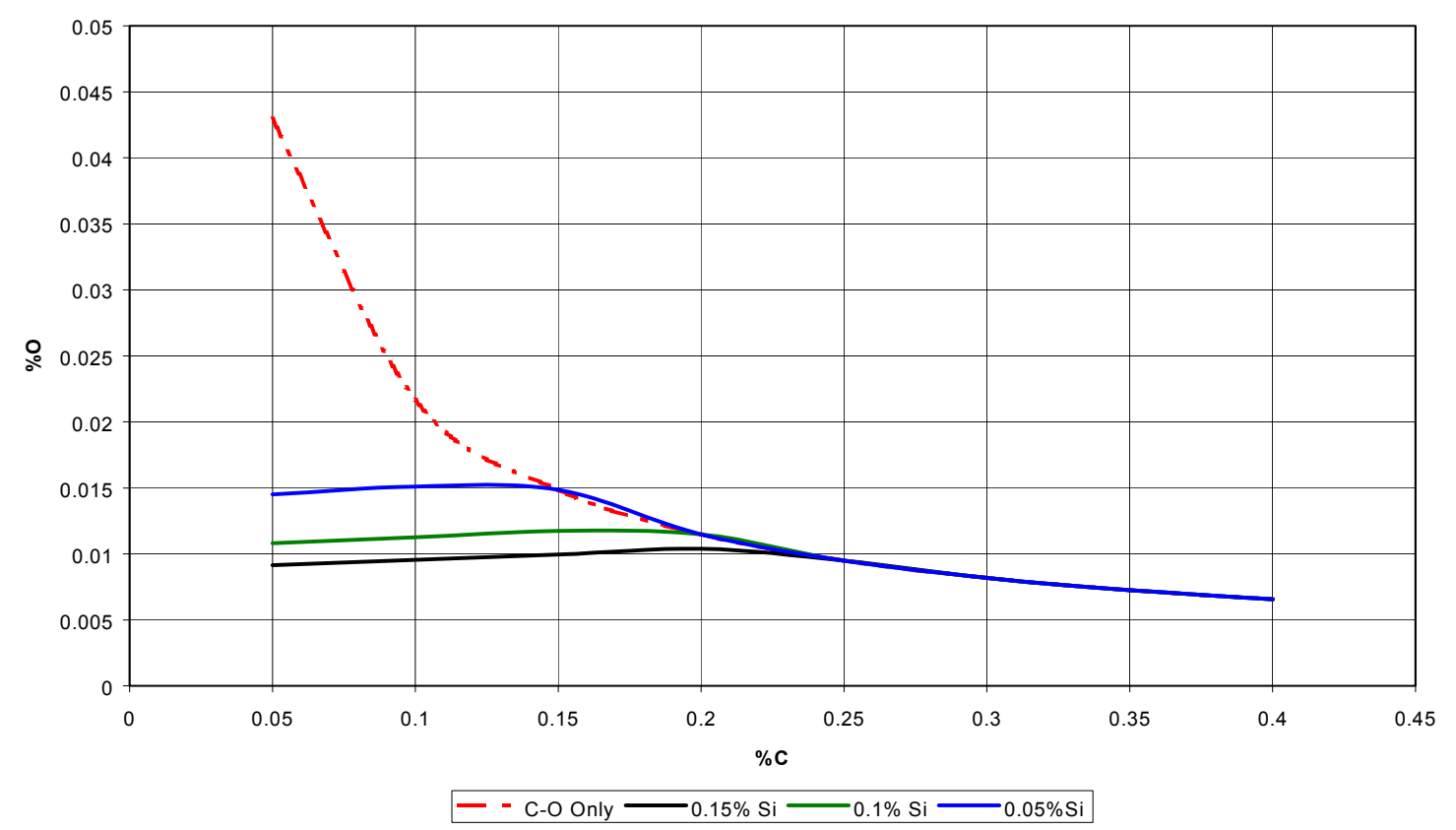

Figure 5. Concentration of oxygen as a function of carbon and silicon level - 1650C, $0.075 \% \mathrm{Mn}$. 
$1600 \mathrm{C}, .075 \% \mathrm{Mn}$

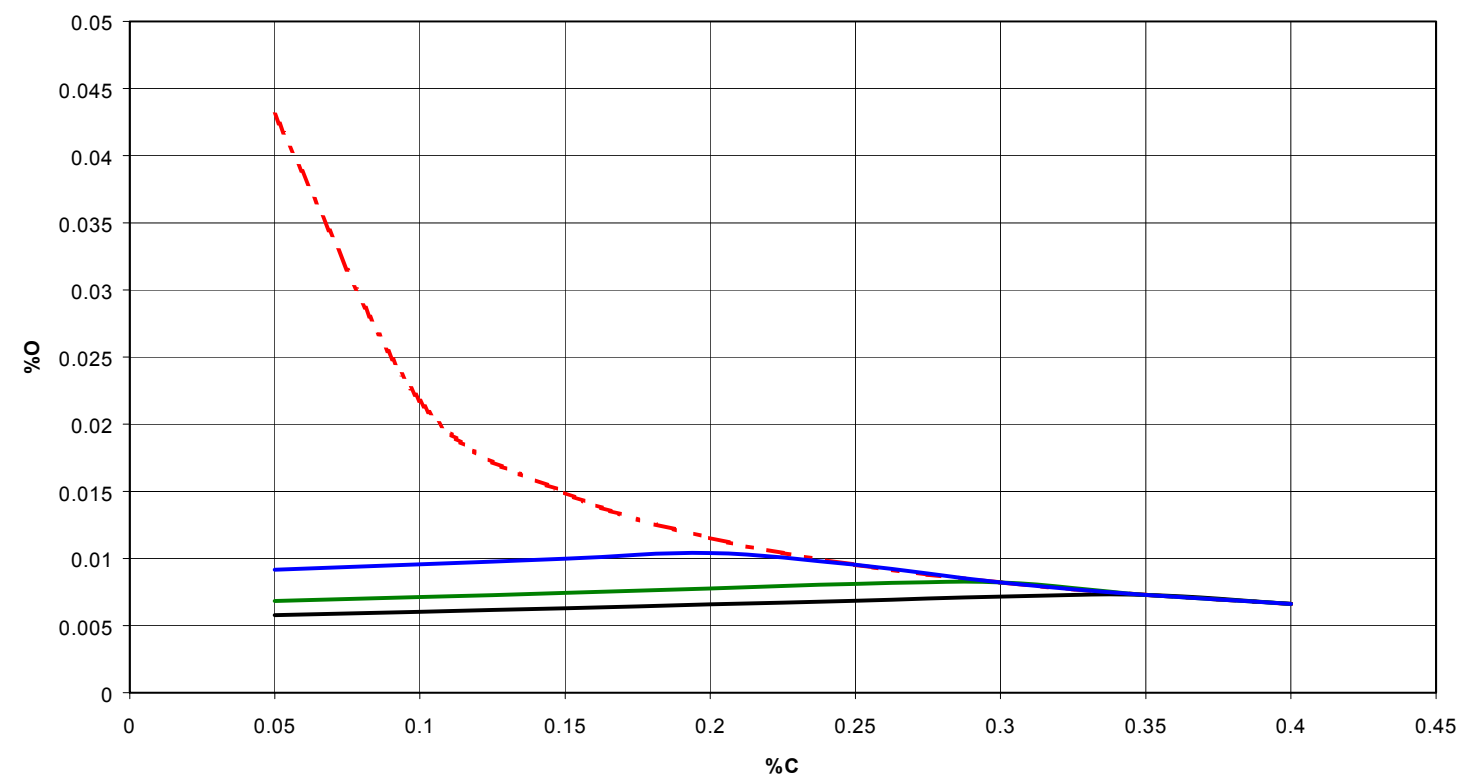

- - C-O Only $-0.15 \% \mathrm{Si}-0.1 \% \mathrm{Si}-0.05 \% \mathrm{Si}$

Figure 6 . Concentration of oxygen as a function of carbon and silicon level - 1600C, $0.075 \% \mathrm{Mn}$.

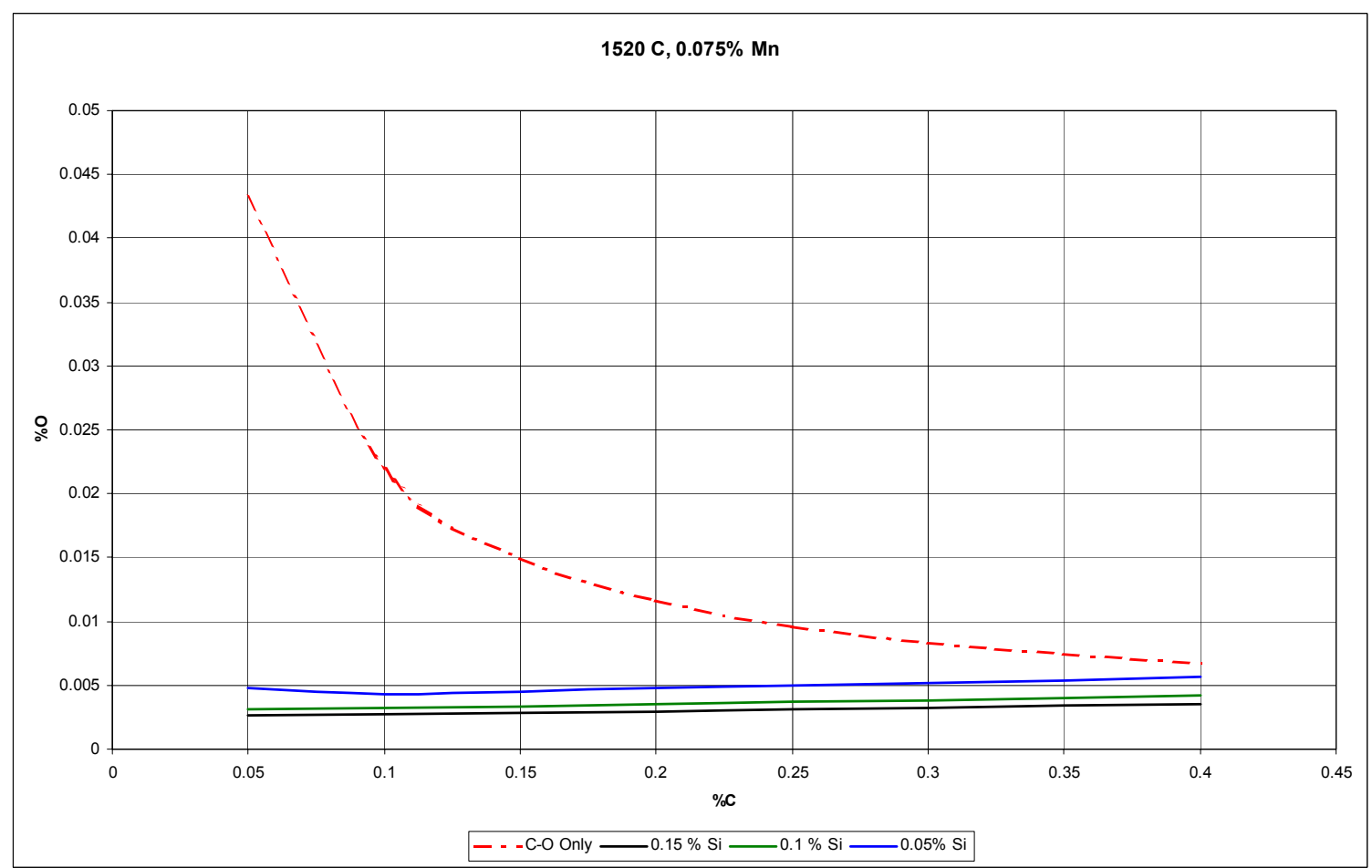

Figure 7. Concentration of oxygen as a function of carbon and silicon level - 1520C, $0.075 \% \mathrm{Mn}$. 


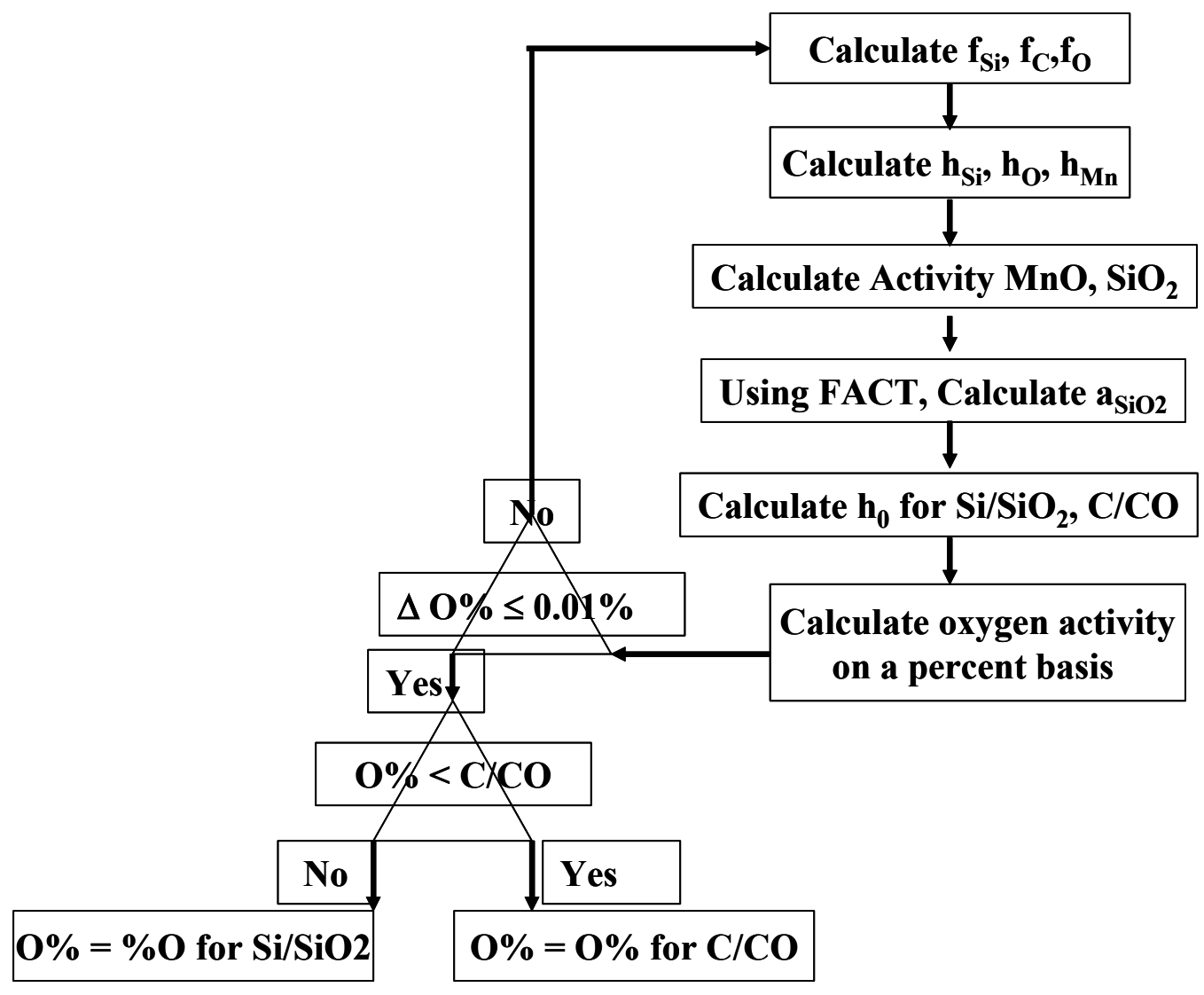

Figure 8. Summary of calculation method for solubility curves.

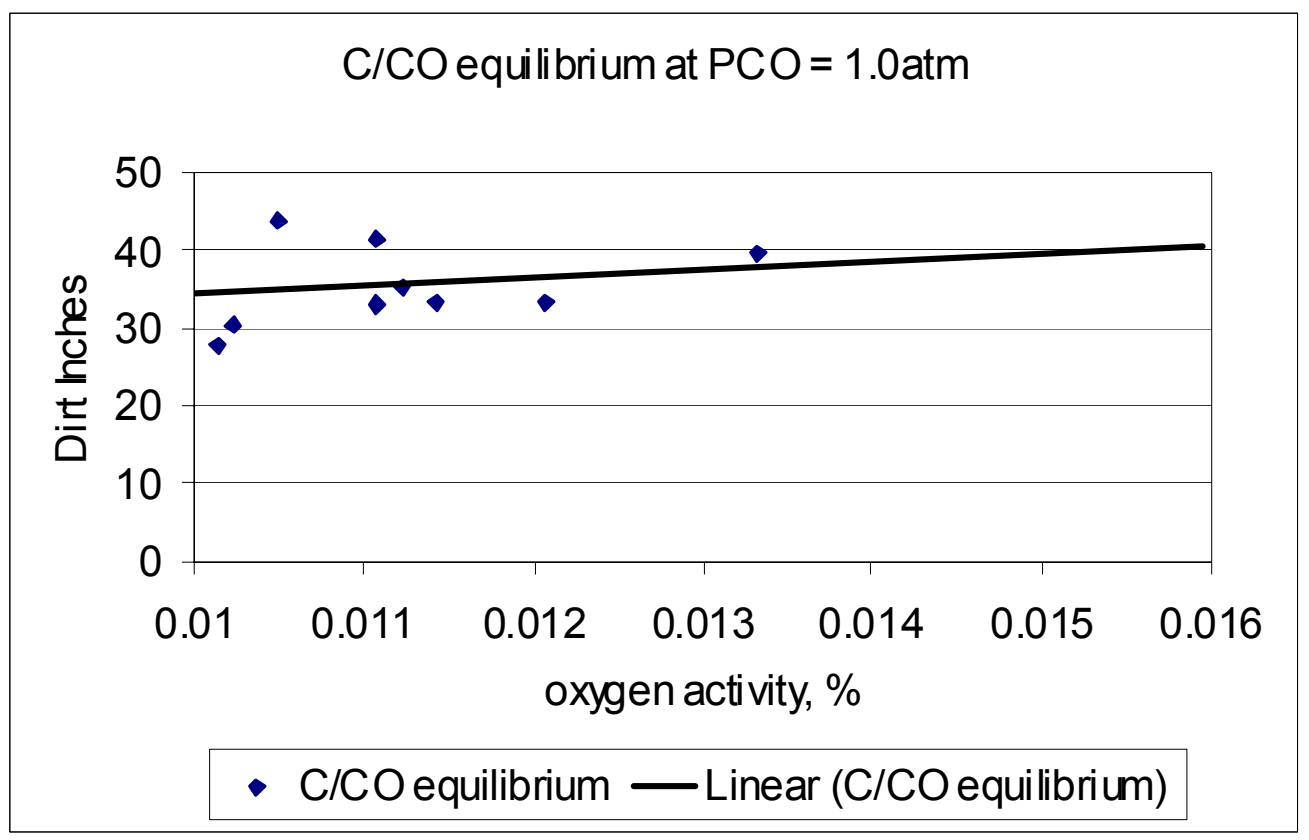

Figure 9. Oxygen activity versus dirt inches. 


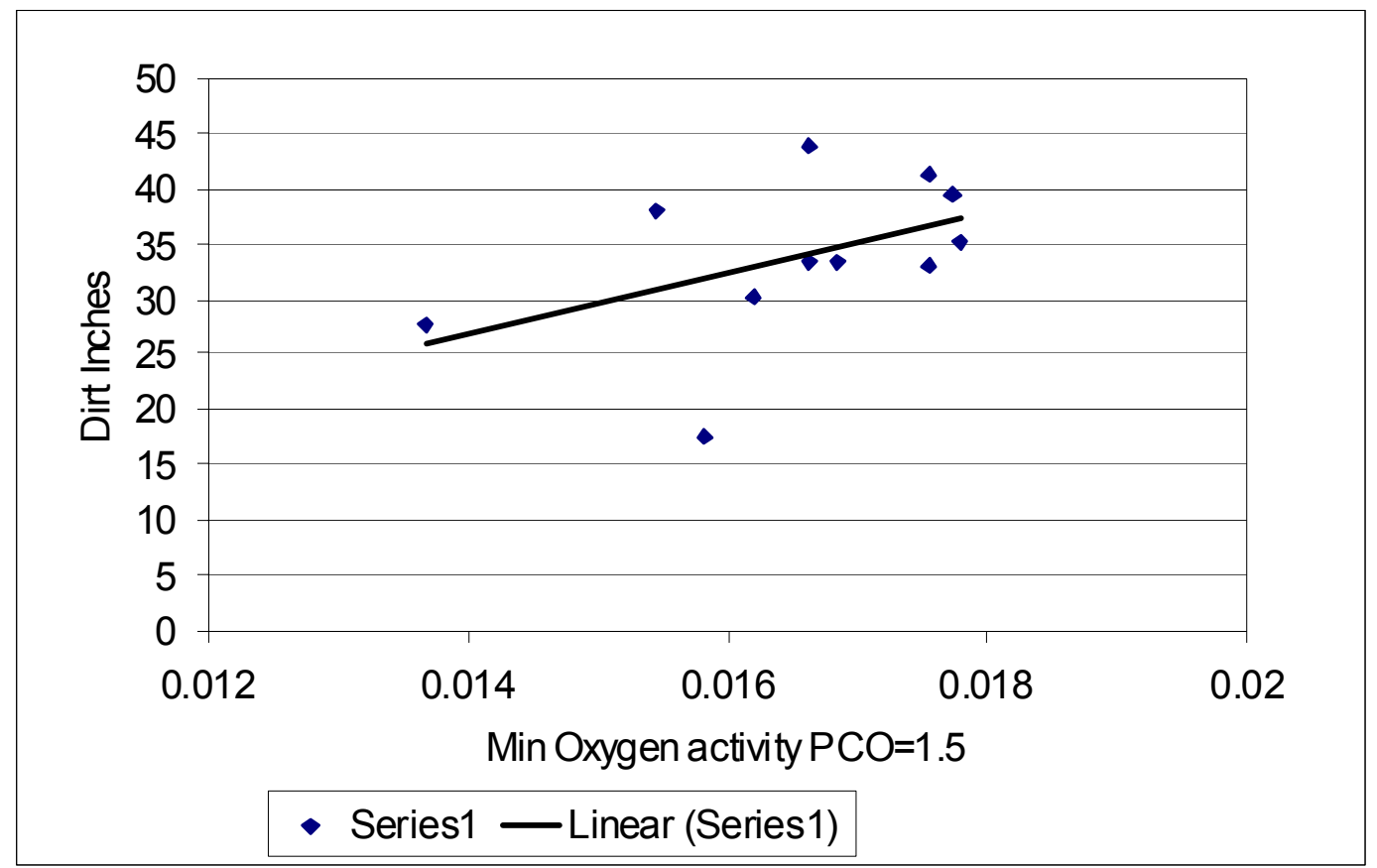

Figure 10. Plot of oxygen activity versus dirt inches with mixed control.

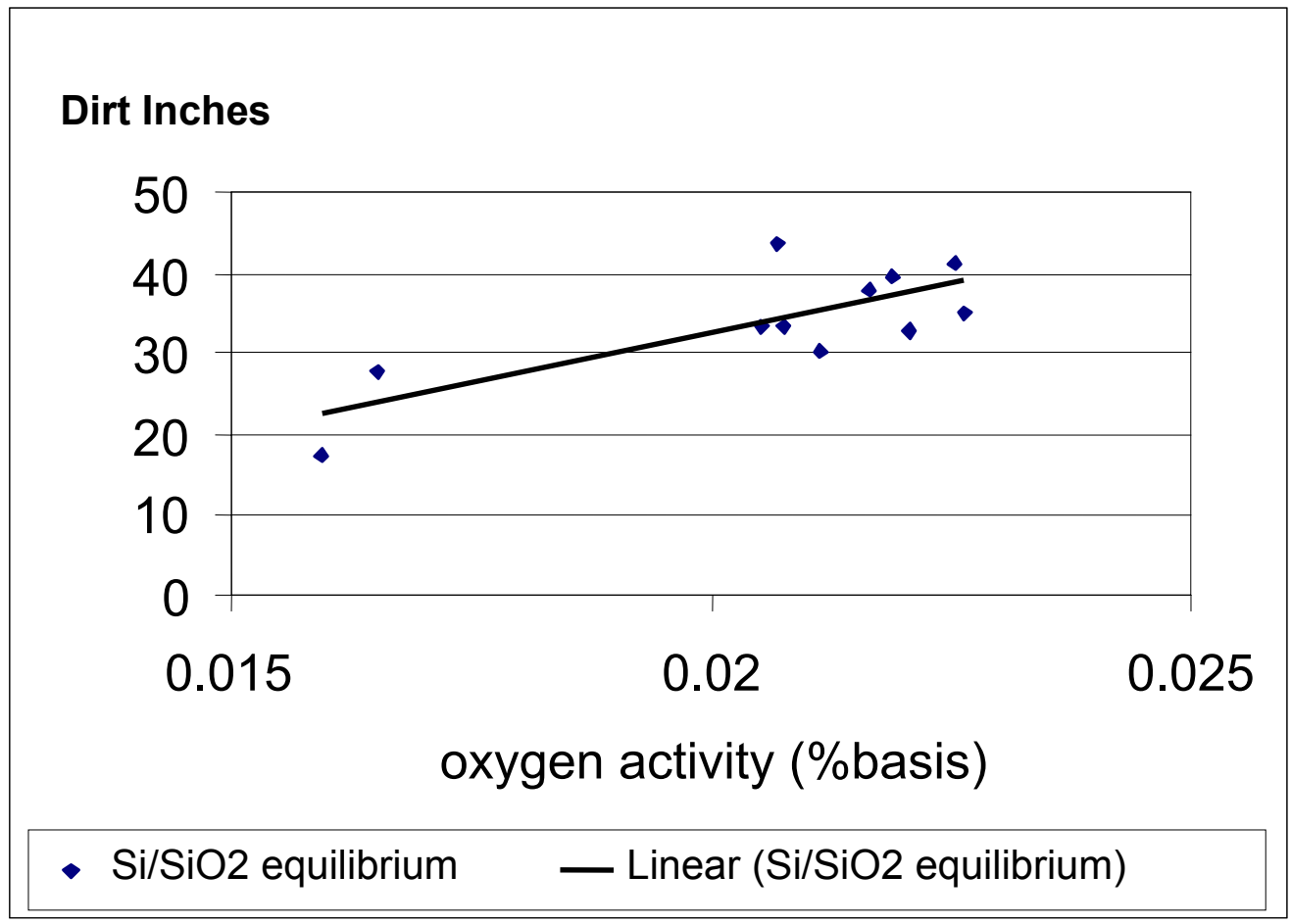

Figure 11. Oxygen activity versus dirt inches with $\mathrm{Si} / \mathrm{SiO}_{2}$ control. 
An oxygen probe was used with each heat, to measure active oxygen. Some calculations were made to determine if the model results correlated with measured oxygen concentrations. The conclusion was that the probe electronics was inadequate at low oxygen concentrations to accurately measure the active oxygen concentrations. At low oxygen concentrations, the oxygen surrounding the probe is reacted and forms a low oxygen zone. This produces a false low oxygen reading.

The model indicates that the $\mathrm{C} / \mathrm{CO}$ equilibrium tends to govern the oxygen concentration and the formation of "dirt" inclusions. This may change with higher silicon charge materials and lower $\mathrm{SiO}_{2}$ in slag and/or higher meltdown temperatures. The crossover between $\mathrm{C} / \mathrm{CO}$ and $\mathrm{Si} / \mathrm{SiO}_{2}$ equilibrium controls may be false due to the use of ladle slag rather than furnace slag compositions rather than furnace slag compositions or the use of acid model rather than a basic model.

At this point, the mathematical model only worked for foundries using an acid slag practice $\left(\mathrm{SiO}_{2} / \mathrm{MgO}\right.$ ratios greater than 0.5). A schematic illustrating the composition range covered by this model is illustrated in Figure 12. A large number of steel foundries use a basic slag practice ( $\mathrm{SiO}_{2} / \mathrm{MgO}$ ratios less than 0.5$)$, so the next goal was to modify the model to allow predictions in basic slags. The slag composition range where the model should apply is illustrated in Figure 13.

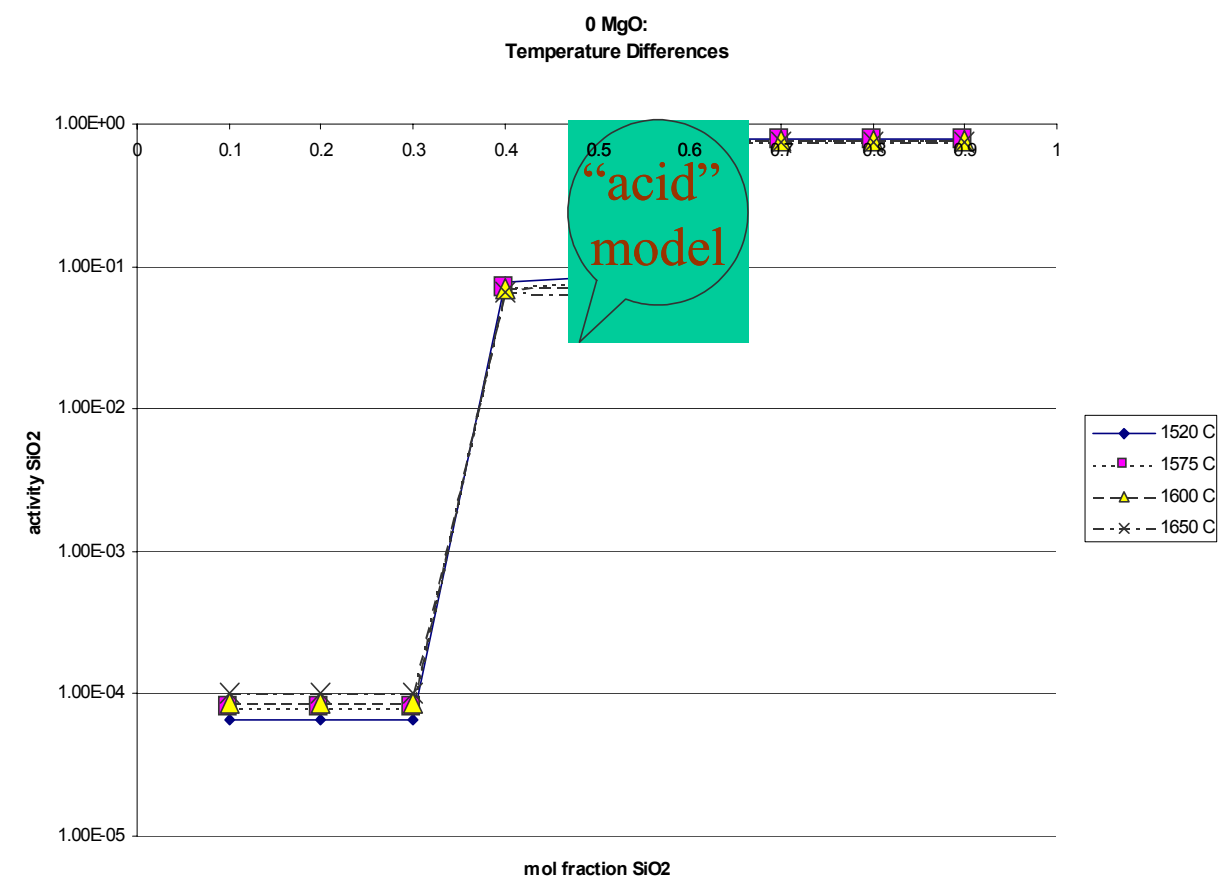

Figure 12. Schematic illustrating chemistry area covered by the acid model. 


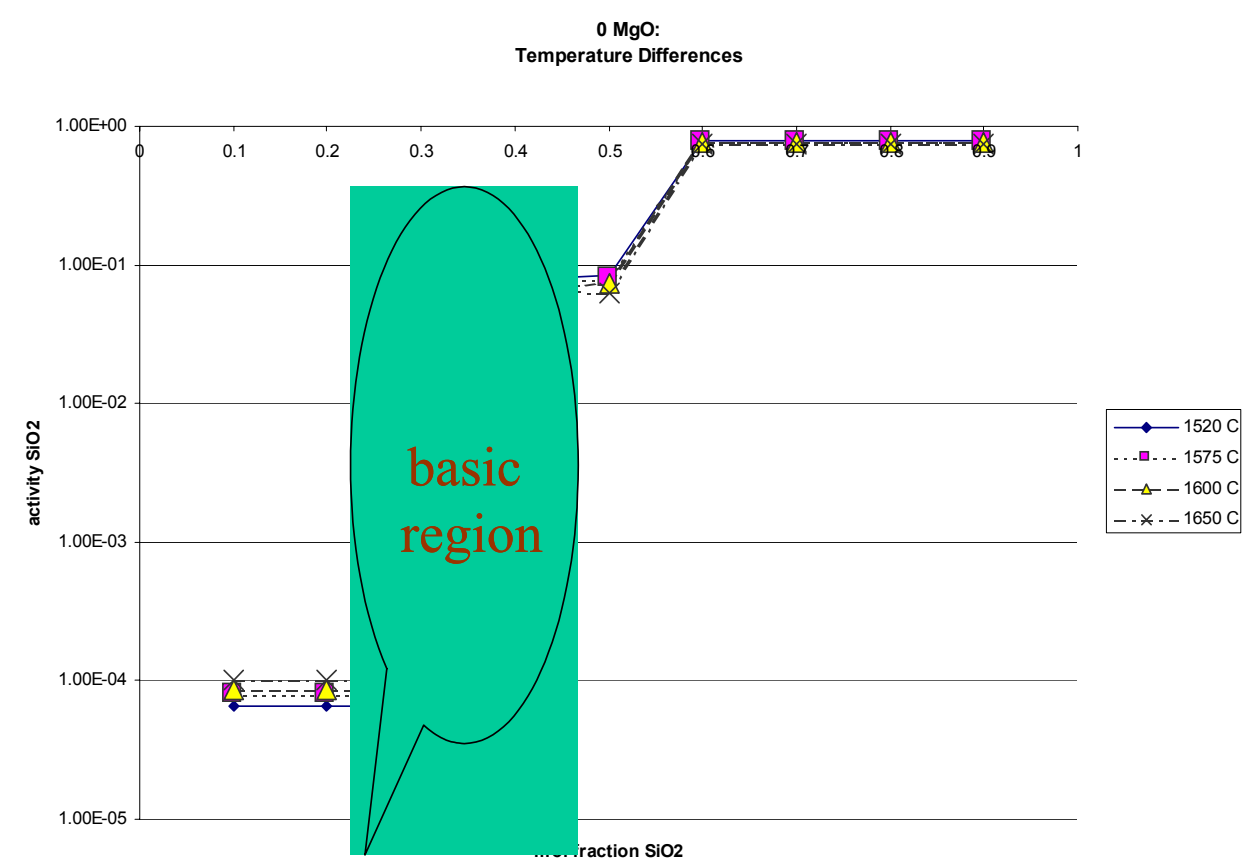

Figure 13. Schematic illustrating chemistry area covered by the basic modification to the model.

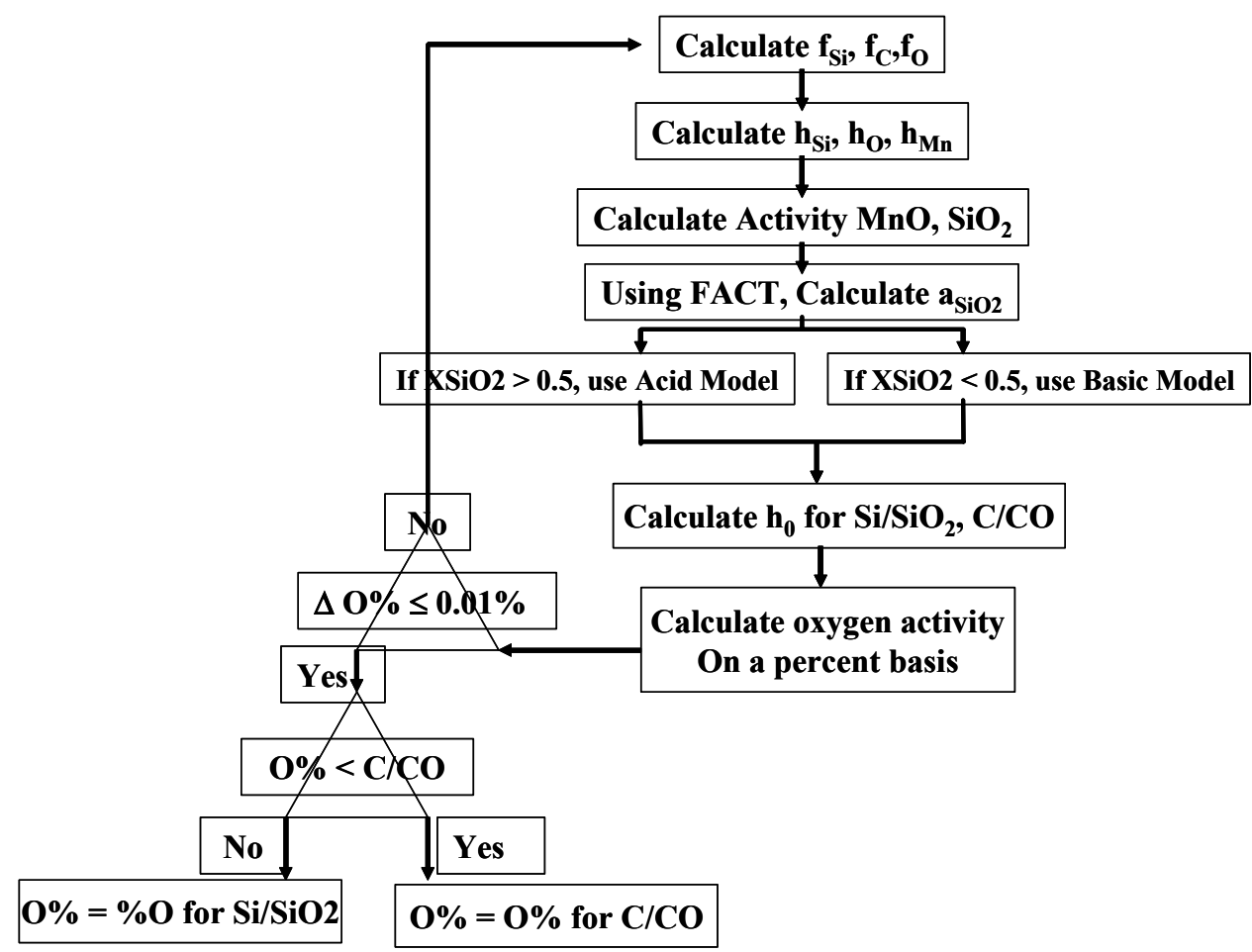

Figure 14. A flowchart of the new acid-basic model. 
There are a number of factors that affect the silica activity in a basic slag. These factors include silica concentration, magnesia concentration, calcia concentration, and temperature. A commercial thermo-chemical activity calculator was used to calculate the silica activity at various temperatures over the composition range in a commercial, basic slag foundry. Multiple linear regression was used to fit an equation through the calculated silica activities, and different formulas were used to provide the best fit to the data. The best fit was provided by using silica concentration, MgO/CaO ratio, and temperature. The $\mathrm{R}^{2}$ of the equation was greater than $98 \%$.

The model was modified to calculate the $\mathrm{SiO}_{2} / \mathrm{MgO}$ ratio from the slag composition and, depending on the slag basicity, either the acid model or the basic model to calculate the total oxygen activity. A flowchart of the new acid-basic model is illustrated in Figure 14 .

The acid-basic model was used to calculate the oxygen activity and the results compared to the casting quality. The results are shown in Figure 15. The number of defects per ton was directly related to the active oxygen content of the heat. A few experimental heats were made where additions of lime and carbon were used in an effort to reduce the oxygen activity. The additions were successful in producing cleaner heats.

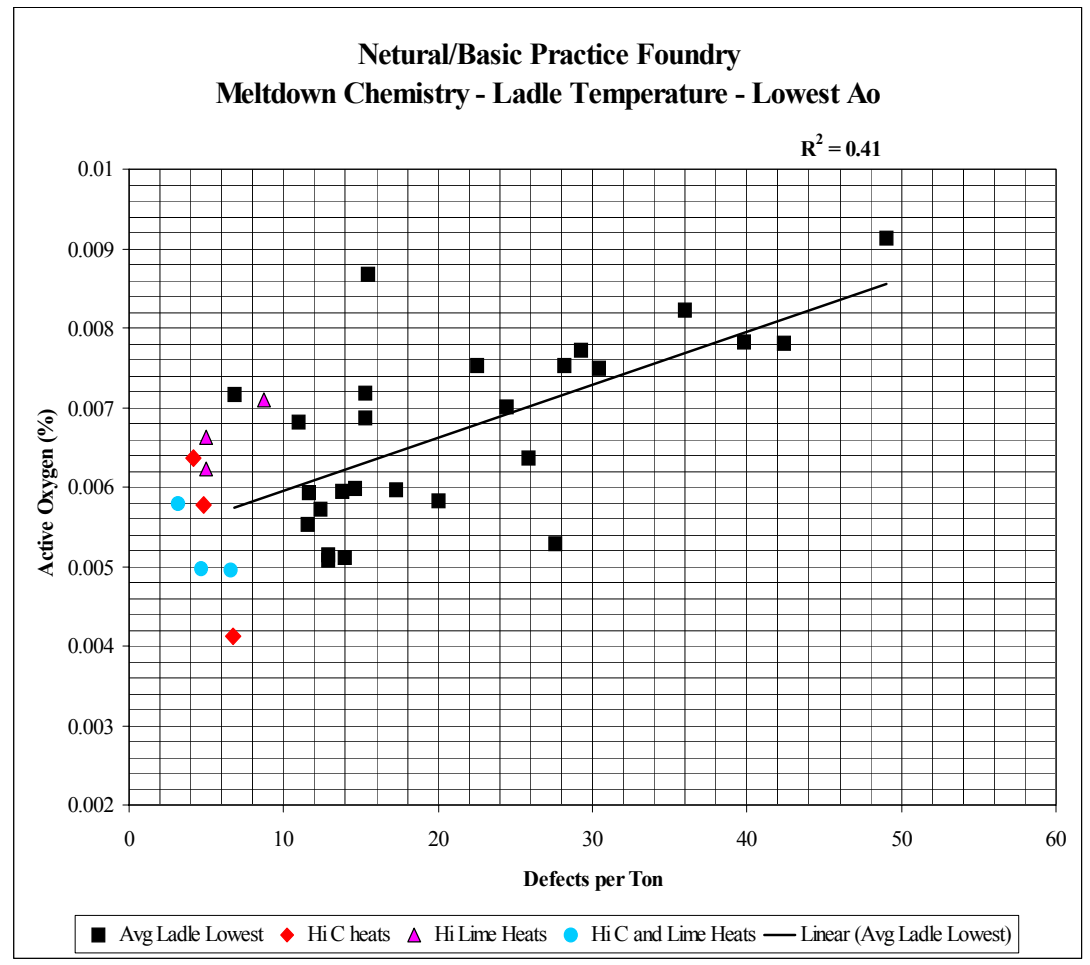

Figure 15. A comparison of calculated oxygen activity and measured casting quality in a basic slag steel foundry. 
A Visual Basic program of the acid-basic slag model to predict a "bad" and "good" heat has been created and given to all steel foundries that requested the program.

\subsection{Summary}

1. An "acid" slag foundry found that higher silicon and manganese residuals after boil/before block produced cleaner heats.

2. A higher bath temperature after the boil increased the silicon and manganese concentrations by disassociation of $\mathrm{SiO}_{2}$ and $\mathrm{MnO}$ in the slag.

3. The $\mathrm{Si} / \mathrm{SiO}_{2}$ equilibrium is more temperature dependent than the C/CO equilibrium.

4. At higher bath temperatures, there is a greater likelihood of $\mathrm{C} / \mathrm{CO}$ control and least a shift in $\mathrm{SiO}_{2}=\underline{\mathrm{Si}} \div+\underline{\mathrm{O}}_{2}$ equilibrium, which raises the Si concentrations while the oxygen limited by $\mathrm{C} / \mathrm{CO}$ equilibrium.

5. The high Si residual acts to buffer the Si control of oxygen so that the $\mathrm{Si}_{\mathrm{SiO}}$ equilibrium for oxygen concentration is reached as the bath cools in later processing.

6. The $\mathrm{Si}_{\mathrm{SiO}}$ equilibrium controls oxygen activity at carbon concentrations of less than about $0.25 \%$ during meltdown and blow.

7. $\mathrm{Si} / \mathrm{SiO}_{2}$ controls oxygen at lower temperatures and higher carbon concentrations.

8. At carbon concentrations of higher than about $0.25 \%$, the C/CO equilibrium controls oxygen activity. (This is only true at the higher temperatures such as at boil.)

9. MnO reduces oxygen activity by combining with $\mathrm{SiO}_{2}$ in the slag. It is beneficial to have less $\mathrm{SiO}_{2}$ in the slag than $\mathrm{MnO}$.

10. Higher Si concentrations after block results in lower oxygen activity. This correlates well with casting quality. 
11. The oxygen activity in basic slags can be controlled either by carbon or silica, depending on temperature, metal chemistry, and slag chemistry.

12. A model has been developed to calculate oxygen activity in the steel for both acid and basic slags. 
CLEAN CAST STEEL TECHNOLOGY, PHASE IV

FINAL TECHNICAL REPORT

\section{Gating System Modification}

Most liquid metals form oxides during exposure to air. The deoxidizers added to steel, including manganese, aluminum, and silicon are especially prone to forming oxides as the metal flows through air and into the runner and casting cavity. These oxides usually float to the cope surface where they have to be removed by arc-air washing and then the cavity filled with weld metal. Subsurface oxides are particularly aggravating because they are difficult to find and weld repair. During machining, the oxides dull or break the machining tools.

Reoxidation defects are the most prevalent surface anomaly encountered on steel castings, and the metal delivery system (pouring practice, stream size, stream compactness, and the sprue, runner, and ingate dimensions) is largely responsible for these defects. [1-4, 6-11] Previous studies have examined the effects of choke location, choke area, and metal flow area. [1-3, 9-11] Many of the studies reached the conclusion that none of the runner-gate designs produce consistent degrees of casting cleanliness.

Gating systems expected to form large amounts of reoxidation particles, based on AFS gating guidelines, have shown a large amount of data scatter with some castings being quite clean and others having many oxide inclusions. This indicates that factors other than the gate dimensions dominate cleanliness. Traditional gating theory emphasizes keeping the runners full by adjusting runner dimensions after each ingate. However, most gating theories are based on steady-state flow, and steady-state flow rarely exists. The entire pouring event is usually a transient phenomenon.

The purpose of the gating system is to deliver liquid metal into the cavity and establish favorable thermal conditions for solidification. In general, it is desirable to fill the mold as fast as possible to avoid misruns and cold laps. It is also desirable to minimize reoxidation defects because these require removal and welding before the casting can be shipped. The purpose of this study was to use simulations to devise improved and consistent gating rules.

The simulation research could best be accomplished by separating the task into two research objectives: determinating a numeric quality predictor and identifying reoxidation particle flow paths during mold filling. The results from each approach will be presented in the following sections. 


\subsection{Numeric Quality Predictor}

Previous phases of the gating study have focused on devising numerical factors with which to differentiate the characteristics of gating systems. Computer simulations of liquid metal flow provide numerical data that can be interpreted in a number of ways. Unfortunately, much of this information is difficult to relate to processes that may cause the reoxidation defects in the final castings. Most industrial uses of fluid flow and fill simulations do not extend beyond observation of free surface contours and qualitative judgments of casting quality.

The turbulent energy of the liquid metal during mold filling has been proposed to be an indicator of the propensity to form defects. However, liquid surface turbulence is the phenomenon that is directly responsible for the formation of oxides in a casting. The need for an accurate method of quantifying liquid free surface turbulence during flow and fill simulations is a crucial step in casting design and cast metal quality.

The authors have proposed using the liquid free surface area data from flow and fill simulations to predict casting behavior and reoxidation tendency. In particular, the integral of liquid free surface area over the filling time has been shown to be more accurate than other numerical indicators in preliminary trials.

In the current study, the effect of mass flow rate on reoxidation was examined with two gating systems. The gating systems used in the trial were those that produced the widest variance in the free surface area integral. Each of the castings was surface ground and defect analysis was performed.

\subsubsection{Experimental Procedure}

A steel test plate was chosen for the study because of its large horizontal cope surface, compact size, and simple geometry. The horizontal cope surface was crucial to the study because it would allow repeatable measurements of surface anomalies. A dimensioned illustration of one of the castings used in the study is shown in Figure 16. 


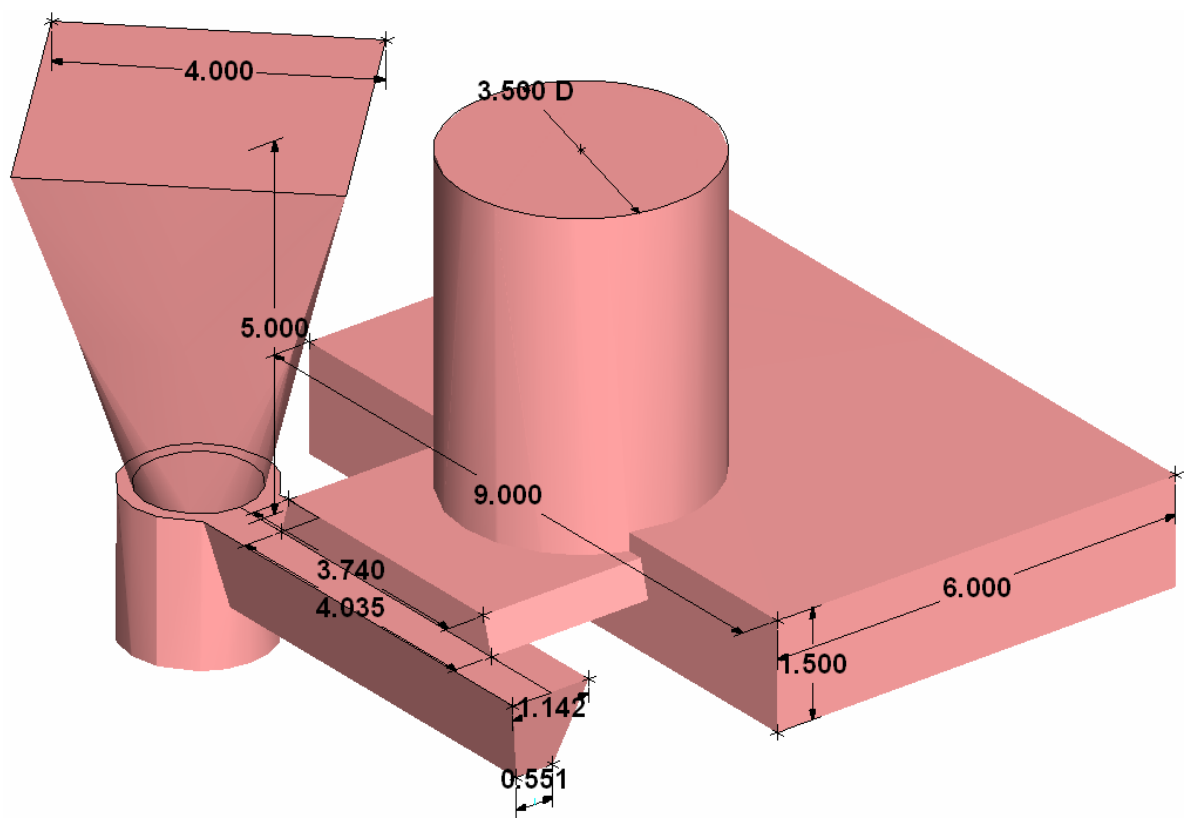

Figure 16. Steel plate geometry used in both the computer simulations and in the foundry trial. The gating system shown above is a 2-1-2 system, which indicates the cross sectional area ratios of the sprue base, runner, and ingate, respectively. All dimensions are in inches.

The three variables in the gating system designed experiment (DoE) were level of metal constraint, runner extension, and cope height. The calculated liquid free surface area and the measured area percent of defects on the cope surface were the dependent variables.

Three other conditions were also evaluated: 1) riser poured plates with a filter, 2) riser poured plates without a filter and 3) a gating system intentionally designed to produce a fill with a high liquid metal surface area and low constraint. The matrix of variables is listed in Table II.

The alloy used for each of the castings was 8630 steel, and all castings were poured at the same foundry. Each casting was surface ground to analyze for reoxidation products, and the total area of these defects was measured using computer-assisted image analysis. 


\begin{tabular}{|c|c|c|c|}
\hline & Constraint & $\begin{array}{c}\text { Runner } \\
\text { Extension }\end{array}$ & Cope Height \\
\hline 1 & ON & OFF & ON \\
\hline 2 & ON & ON & ON \\
\hline 3 & ON & OFF & ON \\
\hline 4 & OFF & OFF & ON \\
\hline 5 & OFF & ON & OFF \\
\hline 6 & OFF & OFF & OFF \\
\hline 7 & OFF ON \\
\hline 8 & ON & ON \\
\hline Riser Pour & Riser Pour With Filter \\
\hline \multicolumn{4}{|c|}{ "Bad" Gating System } \\
\hline
\end{tabular}

Table II. Matrix of variables for the designed experiment (DoE).

\section{1 .2 Results \& Discussion}

Both the simulation results and defect image analysis data for each casting design are listed in Table III. Both the computer simulations and the defect area measurements showed decreased liquid metal reoxidation when filters were added to the riser poured plates. The filters were predicted to reduce the surface turbulence during filling and the results agreed with these predictions. The "bad gating system" also produced the expected results. The "bad gating system" gave the highest liquid free surface area and the highest measured defect area.

The DoE did not produce complete agreement between the predicted and measured results for each variable tested. The computer simulation and measured defect area were in agreement when variations were made in the runner system. There was some disagreement between the simulation predictions and the experimental measurement in the other two DoE experiments. There was no significant response in the measured defect area to the changes in runner constraint even though simulations predicted a reduction in liquid free surface area. The results on the cope height variation were also contradictory. Cope height had the greatest impact on integrated area values obtained from flow and fill simulation and the model predicted that the liquid free surface area should almost double when the cope height was increased but the measured defect area actually went down. 


\begin{tabular}{|c|c|c|}
\hline & $\begin{array}{l}\text { Liquid Free } \\
\text { Surface Area } \\
\text { Integral }\left(\text { in }^{2} \mathrm{~s}\right)\end{array}$ & $\begin{array}{l}\text { Avg. Defect } \\
\text { Area }\left(\text { in }^{2}\right) / \\
\text { Standard Dev. }\end{array}$ \\
\hline Riser Pour with Filter & 73.0 & $/ 0.009$ \\
\hline $\begin{array}{l}\text { Constrained; } 10 " \text { Cope; No } \\
\text { Extension }\end{array}$ & 204.6 & $0.019 / 0.017$ \\
\hline Constrained; 10" Cope & 207.7 & $0.027 / 0.029$ \\
\hline Constrained; No Extension & 137.8 & $0.036 / 0.017$ \\
\hline$" 2-1-2 " ; 10 "$ Cope: No & 252.7 & $0.049 / 0.025$ \\
\hline Extension & & \\
\hline$" 2-1-2 " ;$ 10" Cope & 251.1 & $/ 0.024$ \\
\hline$" 2-1-2 "$ & 162.8 & $/ 0.107$ \\
\hline Riser Pour & 98.7 & $/ 0.092$ \\
\hline "Bad" Gating system & 302.3 & $/ 0.257$ \\
\hline "2-1-2"; No Extension & 159.7 & $0.152 / 0.052$ \\
\hline Constrained & 144.6 & $0.175 / 0.073$ \\
\hline
\end{tabular}

Table III. Results from computer flow and fill simulations as well as defect image analysis results from casting poured in the trial.

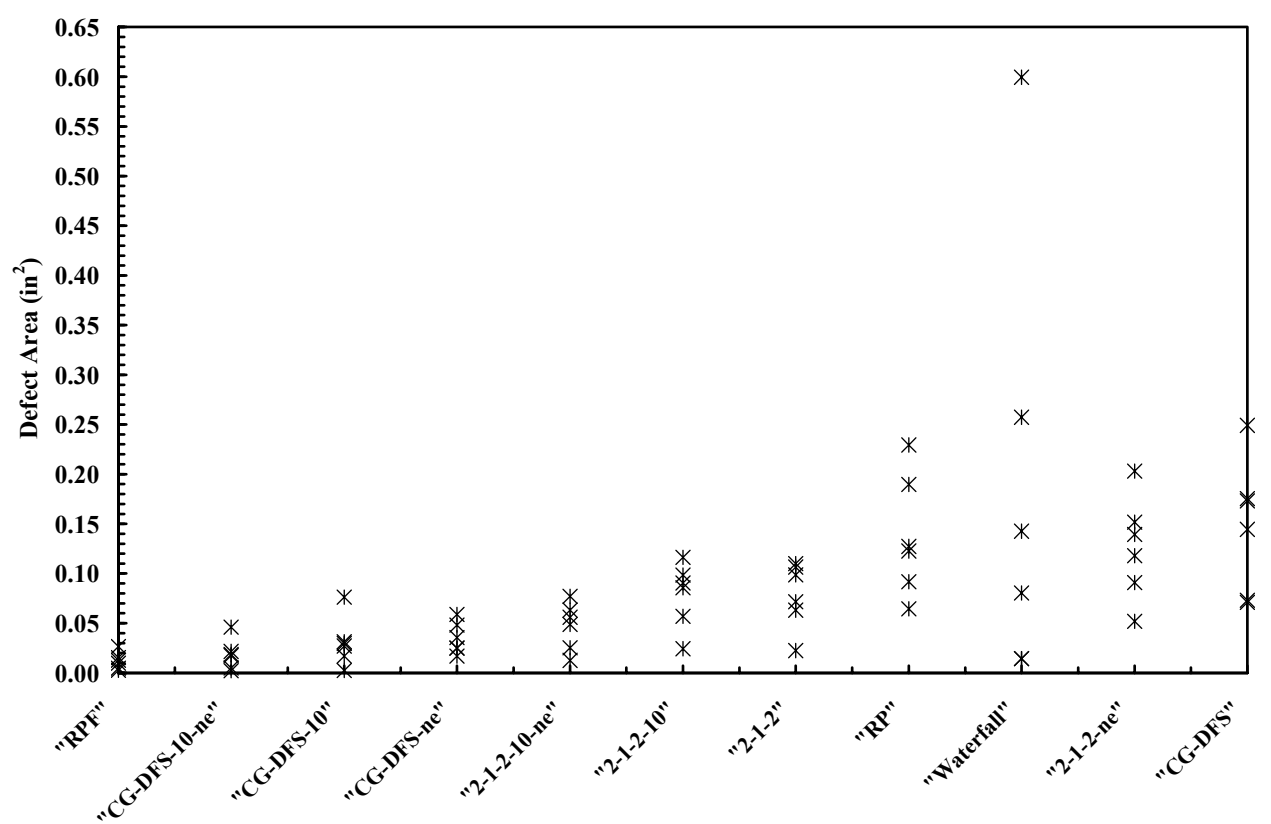

Figure 17. Plot of reoxidation defect area on ground surfaces of cast plates in the trial. 


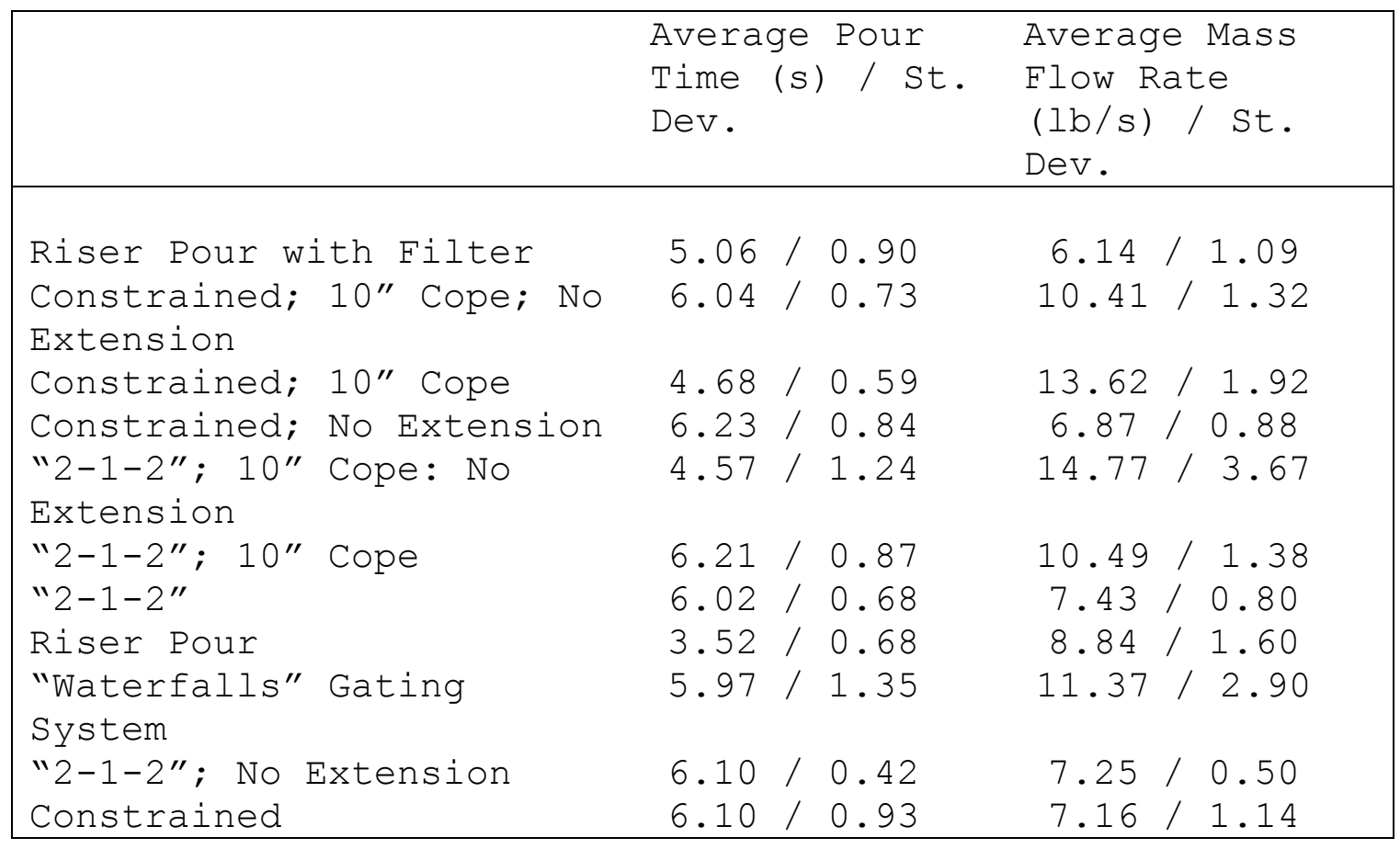

Table IV. Measured pour time and calculated mass flow rate for the castings in the trial.

The data indicated that some other factor was contributing to casting defect formation. The range in the defect area was high in some of the trials and low in others as illustrated in Figure 17. The reasons for this incongruous result became clear after analysis of the pouring times from the casting trials. An analysis of pouring times for each of the castings in the trial revealed that nearly all gating systems were sensitive to pour rate. The measured pour times and mass flow rates for each casting design, listed in Table IV, show that higher mass flow rates produced fewer reoxidation defects in the casting.

Neglecting the filtered riser-pour castings, castings with mass flow rates greater than $10 \mathrm{lbs} / \mathrm{s}$ resulted in relatively few reoxidation defects. The only exception to this finding, the constrained gating system with no runner extension, had a relatively narrow range of mass flow rates, which may have allowed for more consistent behavior. The constrained system with runner extensions - had the lowest volume of reoxidation defects. This gating system was examined in further detail using more specific computer simulations.

Simulation of mold filling indicated that castings having a long pouring time should have higher oxide contents, as much as 90 percent more for some gating systems. A plot of liquid free surface area integral vs. fill fraction for the constrained 
gating system with ten-inch cope height and three different pour times is shown in Figure 18. Doubling the pour time results in more than a doubling of the liquid surface area during mold fill.

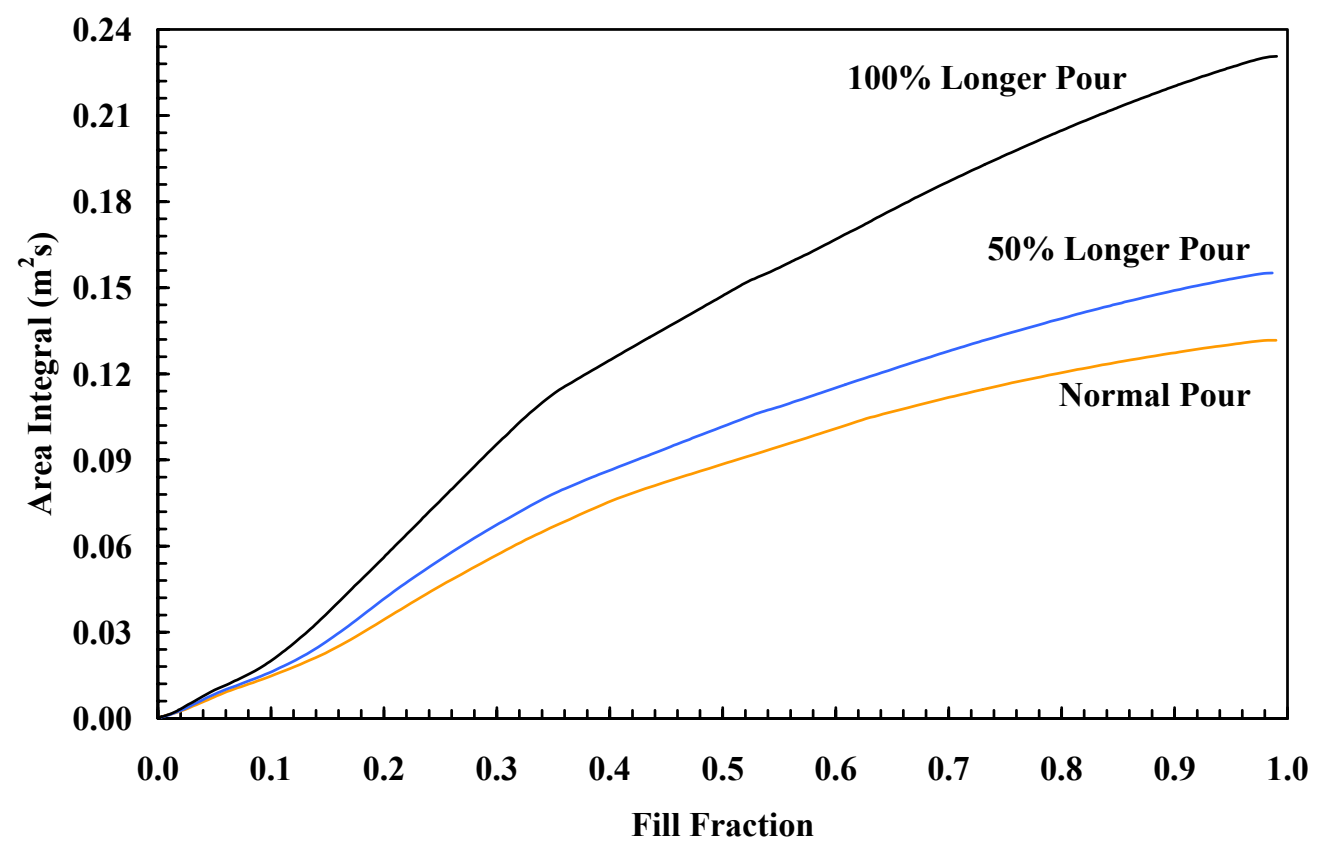

Figure 18. Plot of liquid free surface area generated during mold fill vs. fill fraction for the plate casting with the constrained gating system and 10-inch cope height.

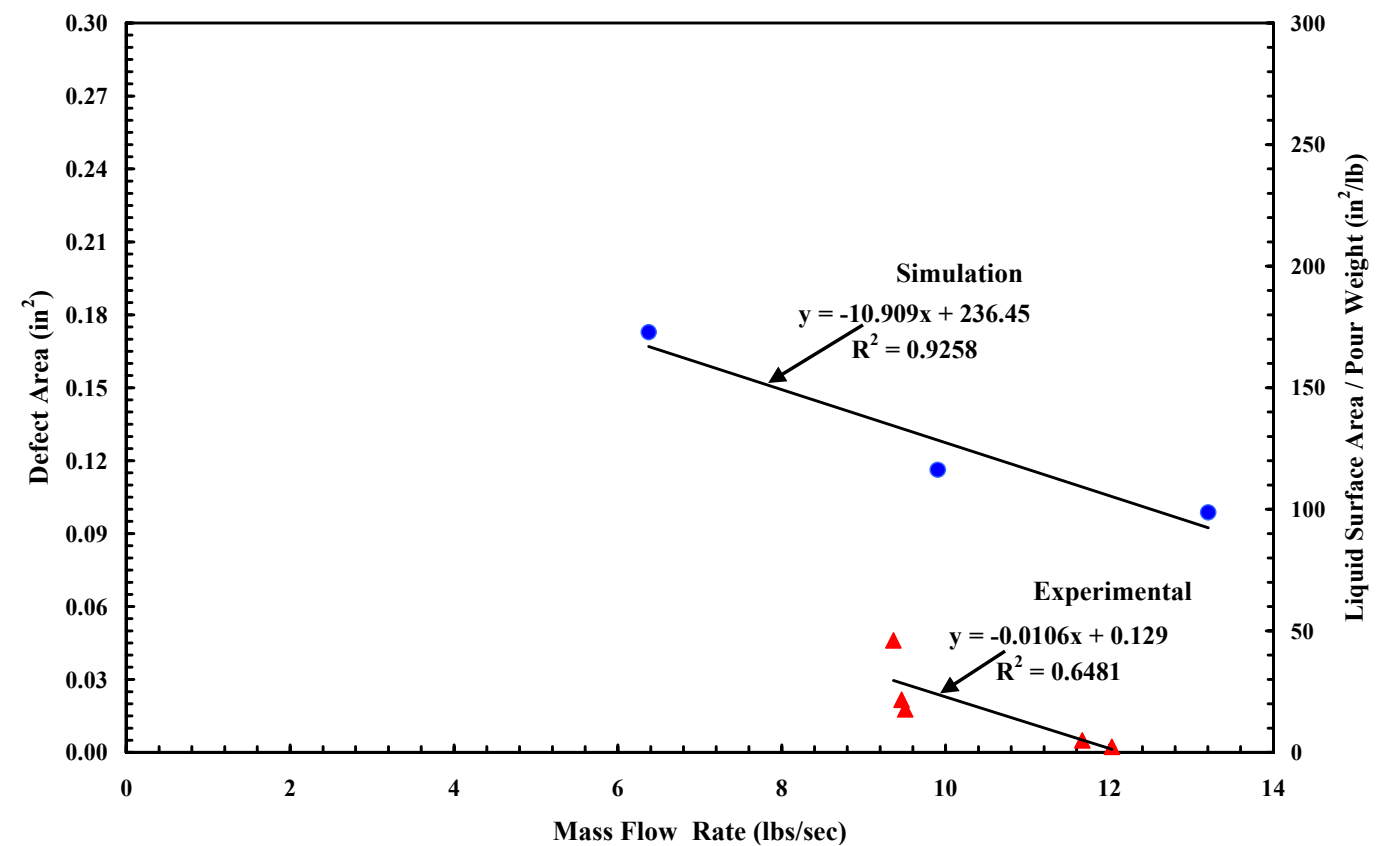

Figure 19. Plot of defect measurements and simulation predictions vs. mass flow rate for the constrained gating system with 10-inch cope height. 


\begin{tabular}{|l|c|c|}
\hline & Experimental & Simulation \\
\hline "2-1-2" & -0.120 & -16.0 \\
\hline $\begin{array}{l}\text { Cope-1-2", } \\
\text { Cope inch }\end{array}$ & -0.017 & -23.0 \\
\hline Constrained & -0.064 & -15.1 \\
\hline $\begin{array}{l}\text { Constrained, 10 } \\
\text { inch Cope }\end{array}$ & -0.011 & -10.9 \\
\hline
\end{tabular}

Table V. Values of slope of linear fit of measured defect area and calculated liquid free surface area vs. mass flow rate.

This analysis also provides a predictor for the effect of a gating system changes on the pouring effect of time. Plots of reoxidation defect occurrence vs. mass flow rate show an inversely proportional relationship with flow rate. An example of these results is illustrated in Figure 19. Computer simulation predictions were plotted on the $y$-axis and the measured defect area on x-axis. A steep slope indicates a high sensitivity to pour time for a specific gating system. Both computer simulations and experimental data show that the linear fits for the constrained gating system are less steep than the "2-1-2" system, which suggests that the constrained system may be more forgiving of pouring time variation as listed in Table $V$.

Liquid-free surface plots also indicate differences in gating system behavior as the pour time varies. Figure 20 illustrates free surface plots colored by pressure for both the constrained gating system and the "2-1-2" gating system; the times shown in the plots are for equivalent fill fractions with each gating system. The constrained system shows an incompletely filled runner in the Figure $20 \mathrm{a}$ but no such region when short pouring times are used (Figure 20b). The "2-1-2" gating system shows a similar partial fill of the runner for the long fill time plot (Figure 20c), but the short fill time plot (Figure 20d) still shows a low pressure region at the runner entrance. This indicates that, even with the best filling conditions, the "2-12" gating system may still generate an appreciable amount of reoxidation defects.

A comparison of the experimental, simulated, and ideal pour times for these castings provided a clear separation of gating system behavior and reoxidation tendency. Figure 21 illustrates pour time vs. pour weight for each casting design. In order to simulate different pour rates, inlet velocities were either increased or decreased from the velocity deemed appropriate to fill a casting. The empirical relationship for pouring time based on pour weight in pounds is as follows:

$$
t=2 / 3 \sqrt{\text { PourWeight }}
$$


Any points that lie below the solid line in the plot would be expected to have very little reoxidation products. As is shown in the plot, very few of the experimental castings had pour times at or below the empirical line or even within 10 percent of the line, as indicated by the two dashed lines. Two of the castings with pour times above this line had few reoxidation defects. Castings with pour times that were much greater than the empirical line exhibited unpredictable reoxidation tendencies and a large scatter in the defect formation. The castings with pour times at or near the empirical line all had the fewest amount of reoxidation. The constrained gating system resulted in fewer reoxidation than the "2-1-2" gating system.

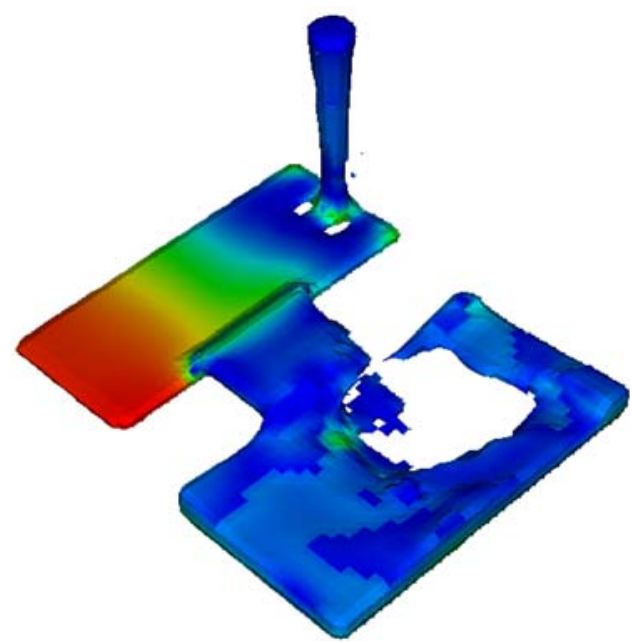

(a)

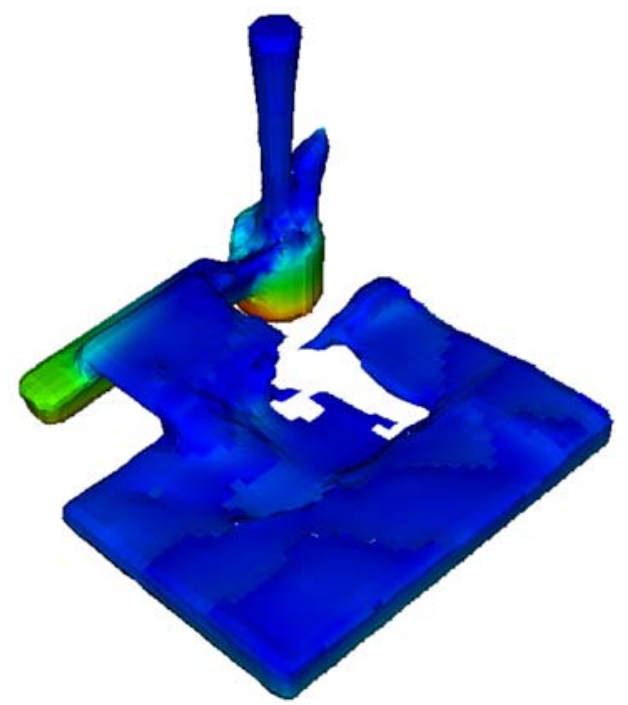

(c)

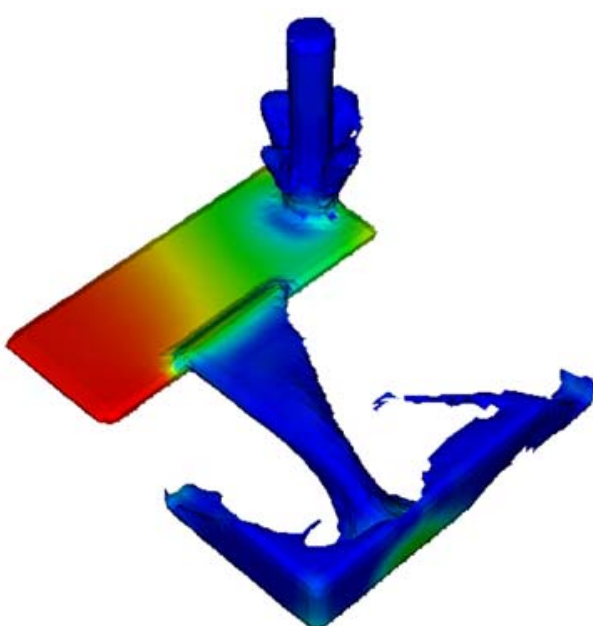

(b)

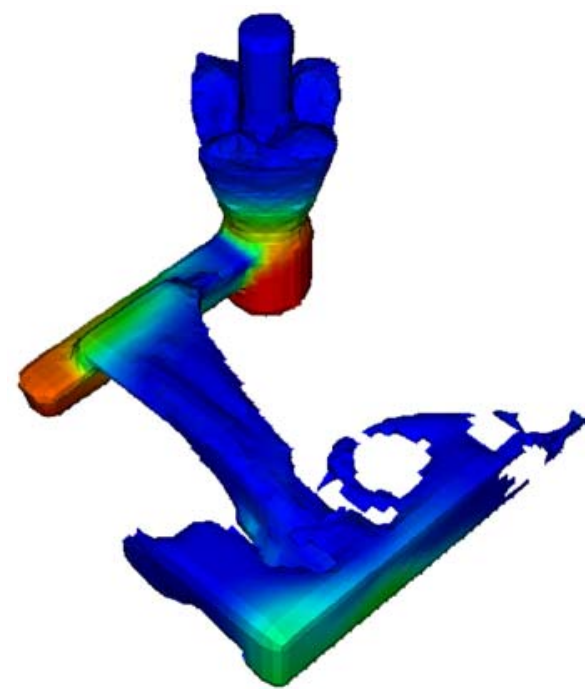

(d)

Figure 20. Isosurface plots of mold fill for the constrained gating system with (a) long fill time, (b) short fill time, and the "2-1-2" gating system with (c) long fill time and (d) short fill time. 


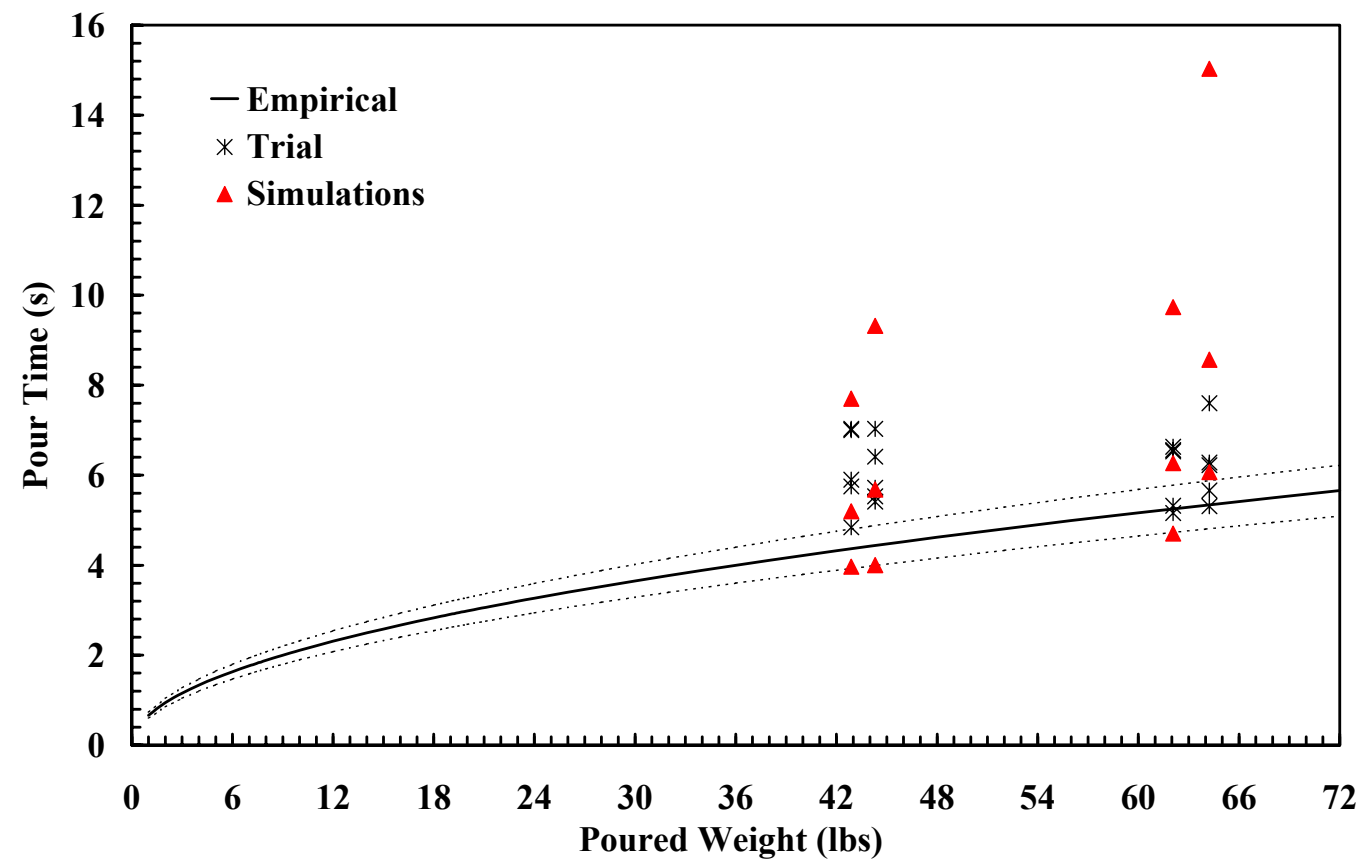

Figure 21. Plot of pour time vs. poured weight for each of the castings in the trial.

\subsubsection{Conclusion}

The effect of pouring time on liquid metal reoxidation during mold filling was examined using computer simulations and plant trials. Initially, it was thought that the liquid free surface area would provide a predictor of casting quality. The mass flow rate was found to have an effect on casting quality, with higher values for mass flow resulting in lower levels of reoxidation. An analysis of pouring time, as compared to the commonly accepted empirical formulation, found that very few of the castings in the trial were actually poured within the ideal time range. When pour times are at or near ideal, castings with a reduced liquid free surface area produced the fewest reoxidation defects. The constrained gating system produced fewer reoxidation defects than the "2-1-2" gating system when pouring was done near the ideal pour time.

\subsection{Flow of Reoxidation Particles}

The liquid metal surface area always correlates with the cleanliness of individual castings with smaller values associated with cleaner castings. However, the large amount of scatter involved made it difficult to differentiate between gating systems even though the simulations predicted that differences 
would exist. [17] Middleton et al. and Scarber reported that taller sprues on a casting resulted in fewer reoxidation defects than shorter sprues. [11, 17] Scarber reported that conditions in the sprue can have a large impact on casting cleanliness and that reoxidation in the sprue may be much greater than in other parts the runner. [21]

There have been numerous studies of gating system design on reoxidation defect formation based on fluid flow analysis. [1015, 19, 20] Many studies have reported inconsistent reoxidation behavior, but changes in the pouring practice for a particular casting - a coupling hub - displayed repeatable reoxidation behavior.[11-13, 15, 17-19] Bottom gating consistently produced about 20 defects per casting, but when the casting was poured through a ceramic shroud, the number of defects was reduced to 1 or 2 per casting. [13]

In the current study, filling of the coupling hub was simulated to determine the fluid flow parameters that might correlate with the casting results. After each simulation, the liquid free surface area was calculated. The integral of the surface area over the fill time is referred to as the Surface Area Integral. The surface area integral is illustrated in Figure 22 for bottom gating and shroud pouring. Shroud pouring reduced the liquid free surface area by about 1/3. Although the trend was in line with experimental observations, the surface area reduction was less than the observed improvement in cleanliness.

Another significant change in reoxidation behavior was observed when reticulated ceramic filters were used in a directpour casting. A 6 inch $\times 9$ inch $\times 1.5$ inch plate casting was filled by pouring directly down the riser to maximize splashing, surface area, and produce as many reoxidation defects as possible. A ceramic filter was placed at the base of the riser sleeve in some molds and similarly poured. The plates with the ceramic filter had an 80\% decrease in reoxidation content, with many of the filtered plates having no reoxidation defects at all. The liquid surface area integral, illustrated in Figure 23, anticipated a 40\% improvement with the use of filters. From these results, it was clear that liquid free surface area did not completely describe events responsible for ceroxide formation.

\subsubsection{Free Surface Velocity}

Flow-3D was modified to allow the liquid surface elements to be segmented into velocity regimes. All of the free surface velocities were calculated in each time step and those in particular velocity ranges were put into velocity "bins". These bins contain the number of surface area elements moving in certain velocity ranges. This produced a surface velocity spectrum such as illustrated in Figure 24(a) for the coupling hub and Figure 24 (b) for the 6 × 9 inch plate. Both the filtered 
plate and the shrouded coupling hub have large amounts of liquid free surface, but the speed is low, less than 0.5 meters per second, which Campbell has recommended as a maximum surface velocity to avoid surface folding. [16]

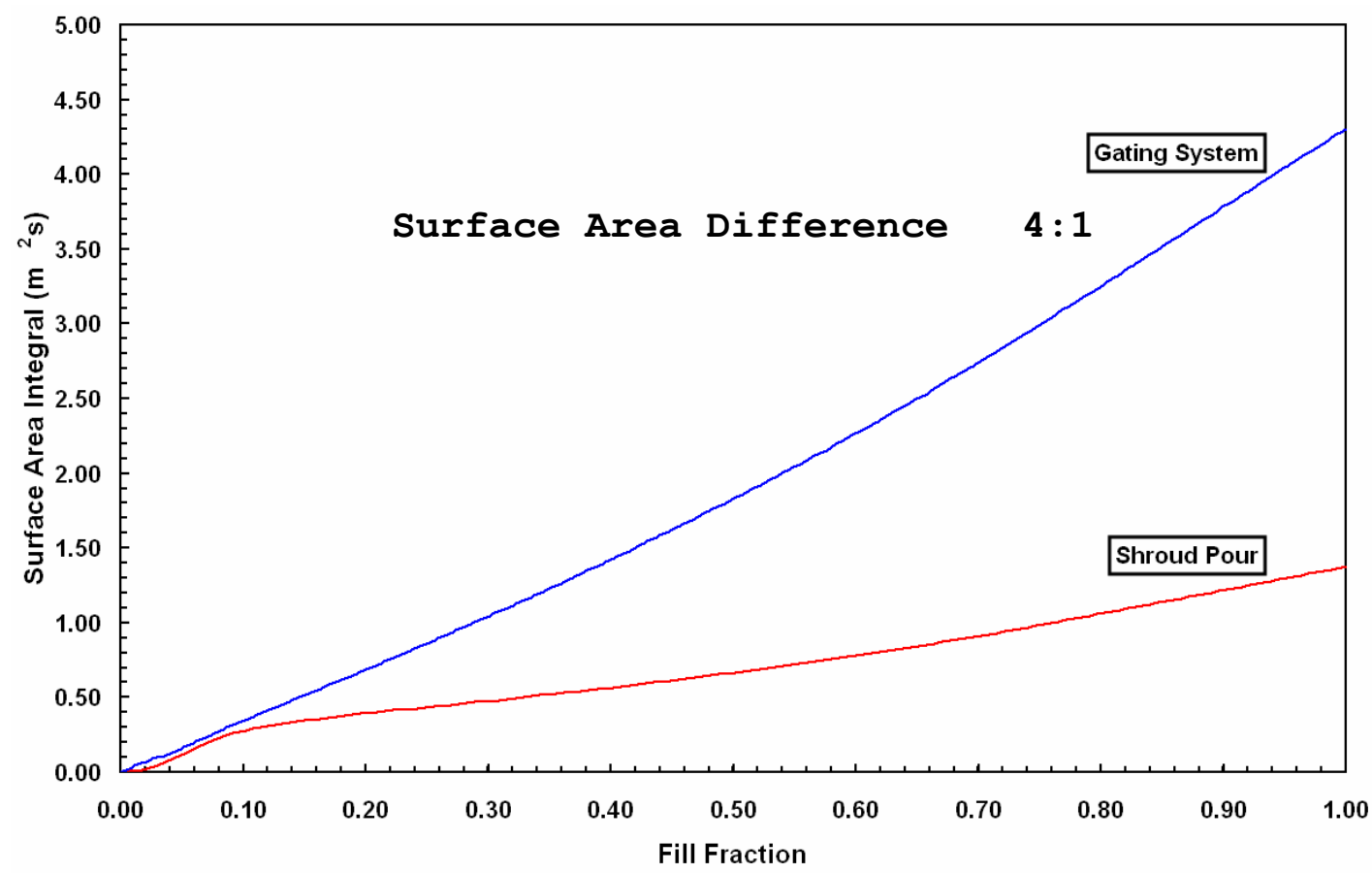

Figure 22. Coupling hub liquid free surface area integral as a function of fill fraction.

In order to summarize the velocity bin plots in a more quantitative fashion, two velocity characterization factors were developed - liquid free surface momentum and liquid free surface kinetic. The liquid free surface momentum is the sum of the product of velocity and the area of liquid surface moving at that velocity for all velocities greater than 0.5 meters per second. The liquid free surface kinetic is the sum of the product of the square of velocity and the area of liquid surface moving at that velocity for all velocities greater than 0.5 meters per second.

The liquid free surface kinetic was the most accurate predictor of reoxidation content in both the coupling hub and in the small plate casting. As illustrated in Figure 24, the ratio of surface kinetic between the bottom-gated coupling hub and the shroud-poured hub was 22-to-1, which is very near what was observed experimentally. Likewise, the ratio was 3.9-to-1 for the small plate casting whereas the experimental observation was 5to-1. Based on these results, a designed experiment was arranged in which the liquid free surface kinetic would be examined more closely. 


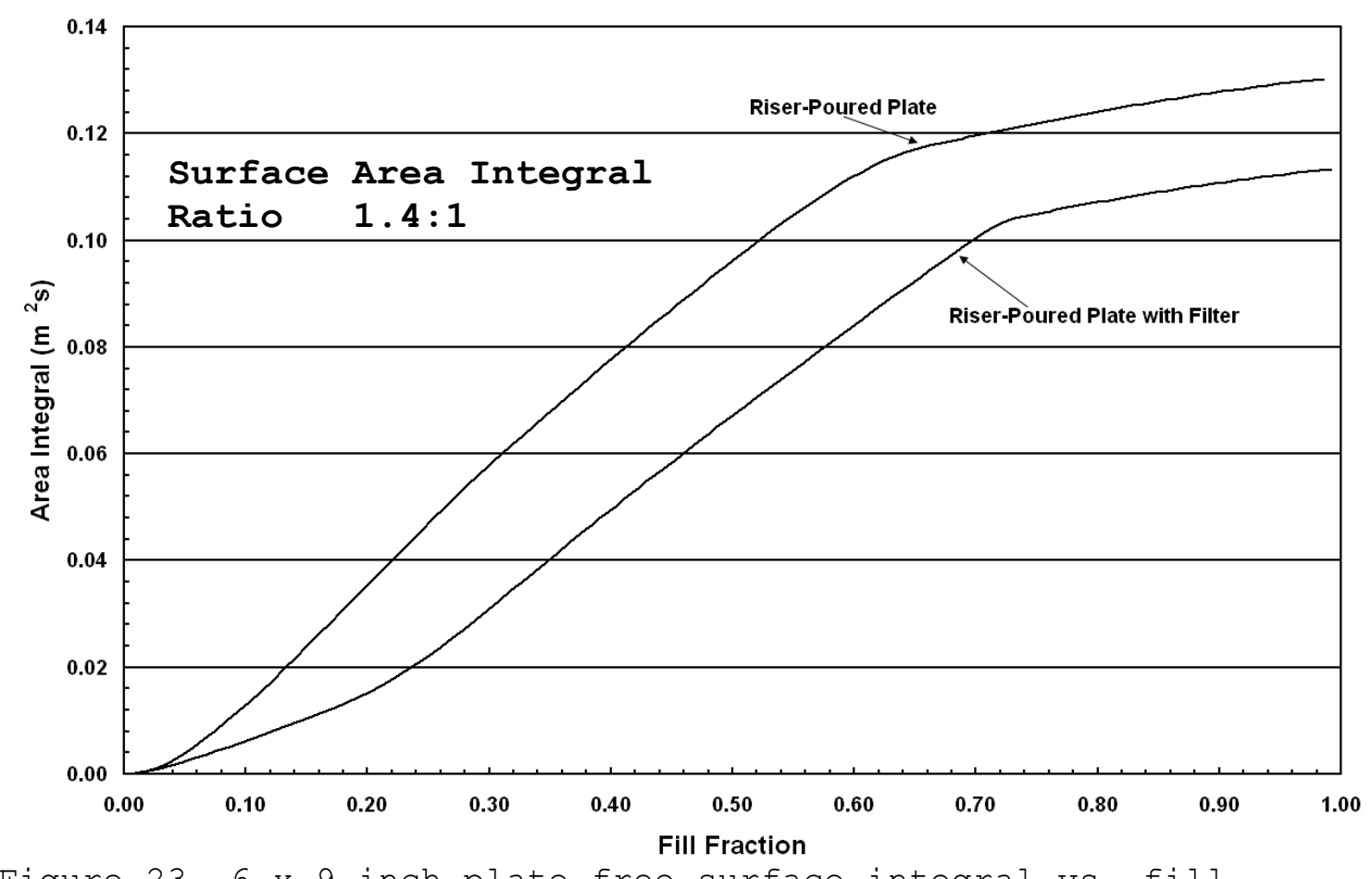

Figure 23. 6 x 9 inch plate free surface integral vs. fill fraction.

\subsubsection{Designed Experiment - Bottom-Gating}

A designed experiment was conducted in which a $6 \times 9 \times 1.5$ inch thick plate casting was poured through a bottom gate having a filter. Pouring basins were used on some molds in an effort to minimize pouring practice effects. All casting geometries are illustrated in Figure 25.

Filters significantly improved casting quality, but no other factor had a significant effect, as detailed in Table VI. The 9-BP casting poured without a basin had a surface momentum of $3.65 \mathrm{~m}^{3} / \mathrm{s}$ and produced 0.45 square inches of oxide defects. Bottom gating with a filter (9-BP2) produced a slightly higher metal surface momentum of 4.04 and slightly higher defect area of 0.47 square inches. Bottom gating with a filter produced a surface momentum of 2.60 and eliminated surface defects. The use of basins always produced more reoxidation defects except when filters were used. The reasons for this are not clear. Surface momentum calculations correlated reasonably well with experimental results. However, basin and filter behavior were always incorrectly predicted in magnitude and trend. 


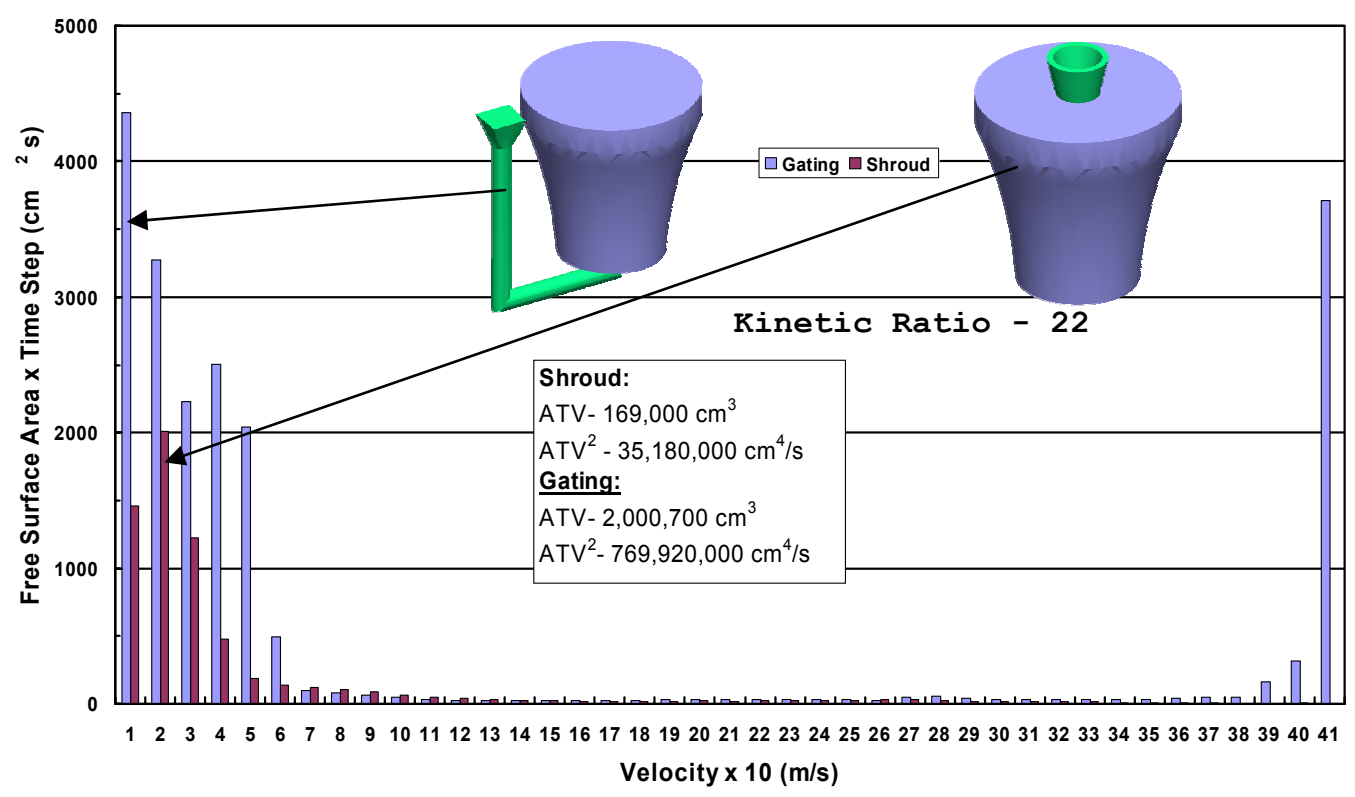

(a)

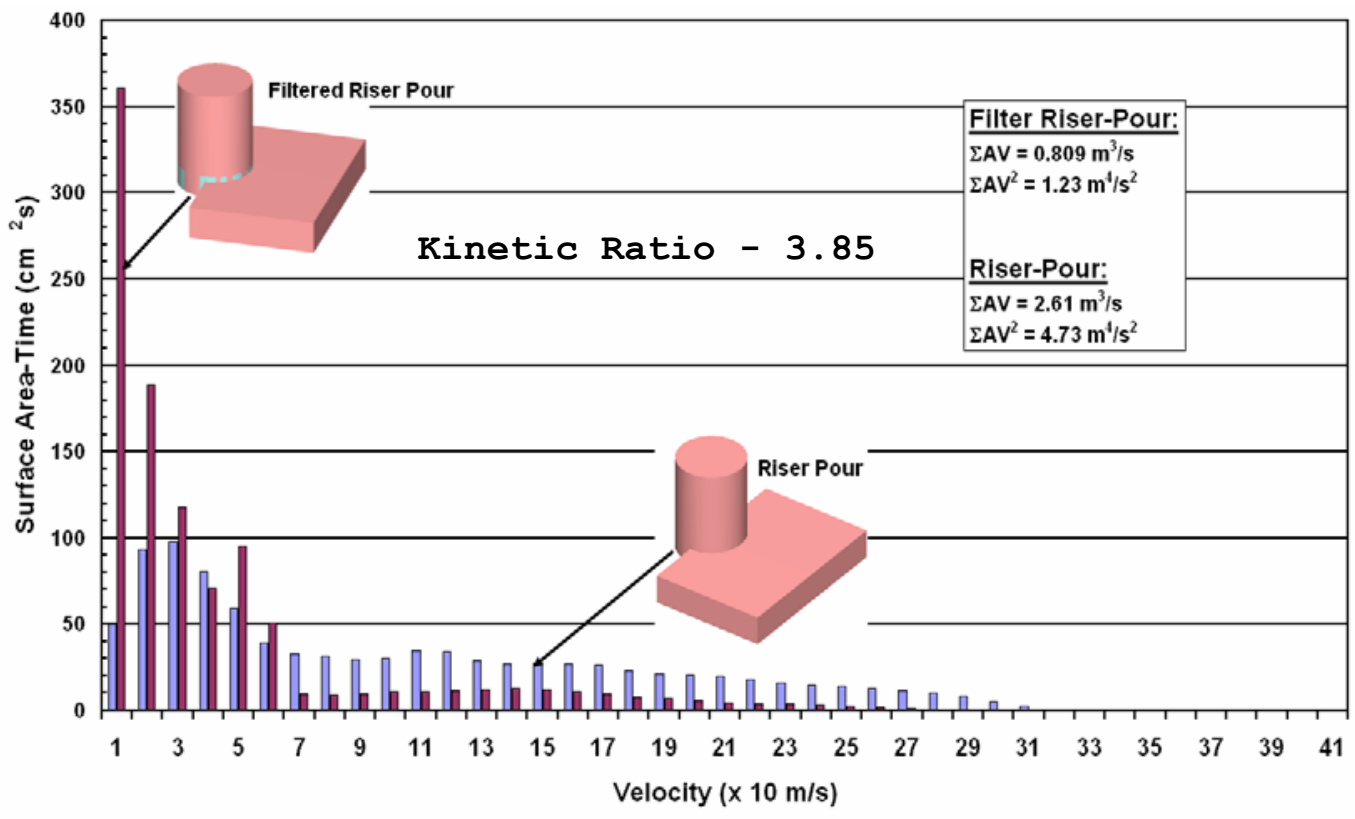

(b)

Figure 24. Velocity bin plots for the (a) coupling hub and the (b) small steel plate. 

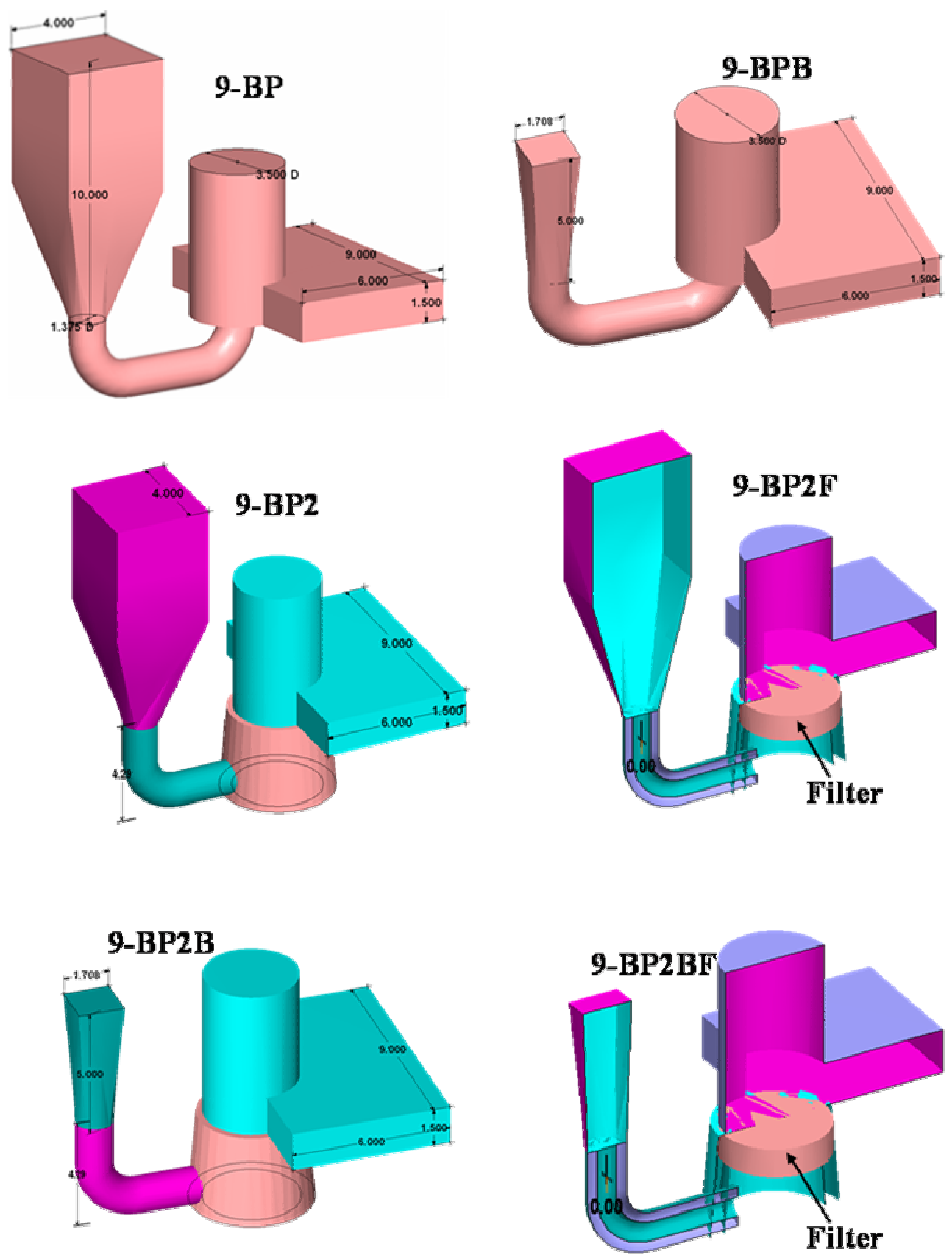

Figure 25. Geometry of castings examined in the designed experiment. All dimensions are in inches. 


\begin{tabular}{|c|c|c|c|c|}
\hline & $\begin{array}{c}\text { Surface } \\
\text { Area } \\
\text { Integral } \\
\left(\mathrm{m}^{2} \mathrm{~s}\right)\end{array}$ & $\begin{array}{c}\text { Surface } \\
\text { Momentum } \\
\left(\mathrm{m}^{3} / \mathrm{s}\right)\end{array}$ & $\begin{array}{c}\text { Surface } \\
\text { Kinetic } \\
\left(\mathrm{m}^{4} / \mathrm{s}^{2}\right)\end{array}$ & $\begin{array}{c}\text { Measured } \\
\text { Defect Area } \\
\left(\text { in }^{2}\right)\end{array}$ \\
\hline \multicolumn{5}{|c|}{ Bottom Gating (9-BP and 9-BPB) } \\
\hline $\begin{array}{r}\text { No } \\
\text { Basin }\end{array}$ & 0.37 & 3.65 & 6.75 & 0.45 \\
\hline Basin & 0.14 & 0.98 & 1.23 & 0.28 \\
\hline \multicolumn{5}{|c|}{ Bottom Gating Without Filter (9-BP2 and 9-BP2B) } \\
\hline $\begin{array}{r}\text { No } \\
\text { Basin }\end{array}$ & 0.38 & 4.04 & 7.64 & 0.47 \\
\hline Basin & 0.10 & 0.42 & 0.47 & 1.53 \\
\hline \multicolumn{5}{|c|}{ Bottom Gating with Filter (9-BP2F and 9-BP2BF) } \\
\hline $\begin{array}{r}\text { No } \\
\text { Basin }\end{array}$ & 0.41 & 2.60 & 4.86 & 0.00 \\
\hline Basin & 0.17 & 0.11 & 0.13 & 0.03 \\
\hline
\end{tabular}

Table VI. Results from simulations and experimental measurements of castings in the designed experiment.

\subsubsection{Theory}

Liquid metal reoxidation occurs with almost no barrier to the reaction and continues until the mold is filled. Reoxidation can occur at any location where metal is exposed to oxygen whether in the mold, gating system, or ladle, or mold binder. Some surfaces will always be present in the mold cavity, so the focus of this discussion will be on reducing oxidation during metal delivery to the metal cavity.

Several investigators have reported that more oxidation occurs in the sprue than in any other part of the mold. [10-13, 16-18] This is a result of air being entrained and reacted with the metal by impingement of metal from the ladle on liquid in the sprue cup or pouring basin.

Simulations were performed to estimate the variability caused by changing the conditions in the sprue. The reactions were minimized by placing an artificial shroud to the $6 \times 9 \times 1.5$ inch plate casting and results were compared to those filled in a conventional manner using AFS gating guidelines. It was found that events in the sprue were responsible for $80 \%$ of the liquid free surface area produced during mold filling. The results obtained in simulations of pouring a casting gated using a 1:2:4 gating ration compared to results obtained with the same ratio and a shroud are illustrated in Figure 26. Pouring through the 
shroud reduced the surface area exposed to air by about $80 \%$.

A metal stream from a teapot ladle impinging on liquid in a sprue cup is illustrated in Figure 27. The circles in the illustration represent reoxidation particles formed in reactions with oxygen and entrained in the metal. The liquid is assumed to fall from height $H$ with negligible horizontal components. The liquid and reoxidation particle properties were assumed to be invariant with temperature.

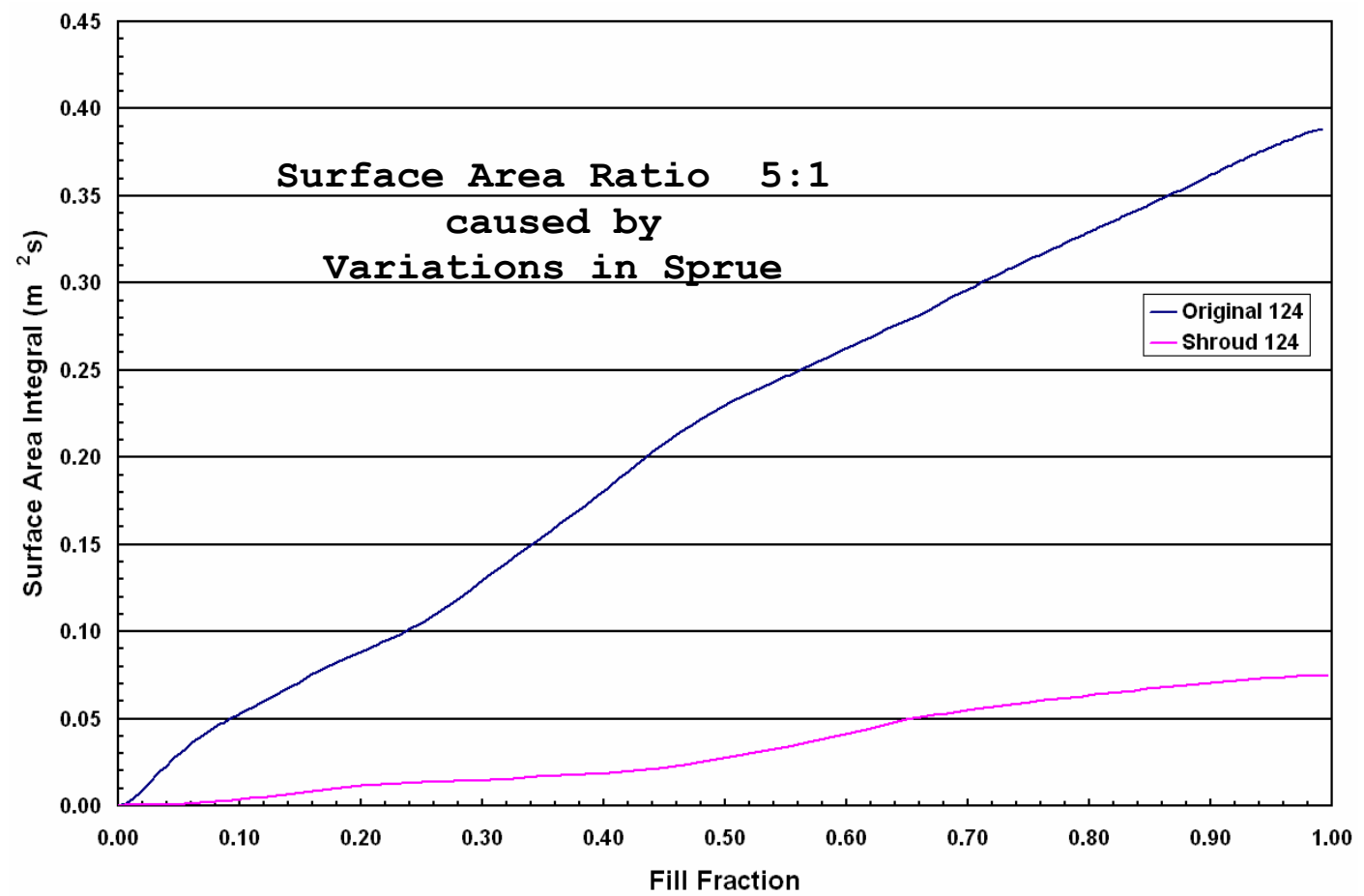

Figure 26. Liquid free surface area integral for conventionally gated casting and similar casting poured through a shroud. 


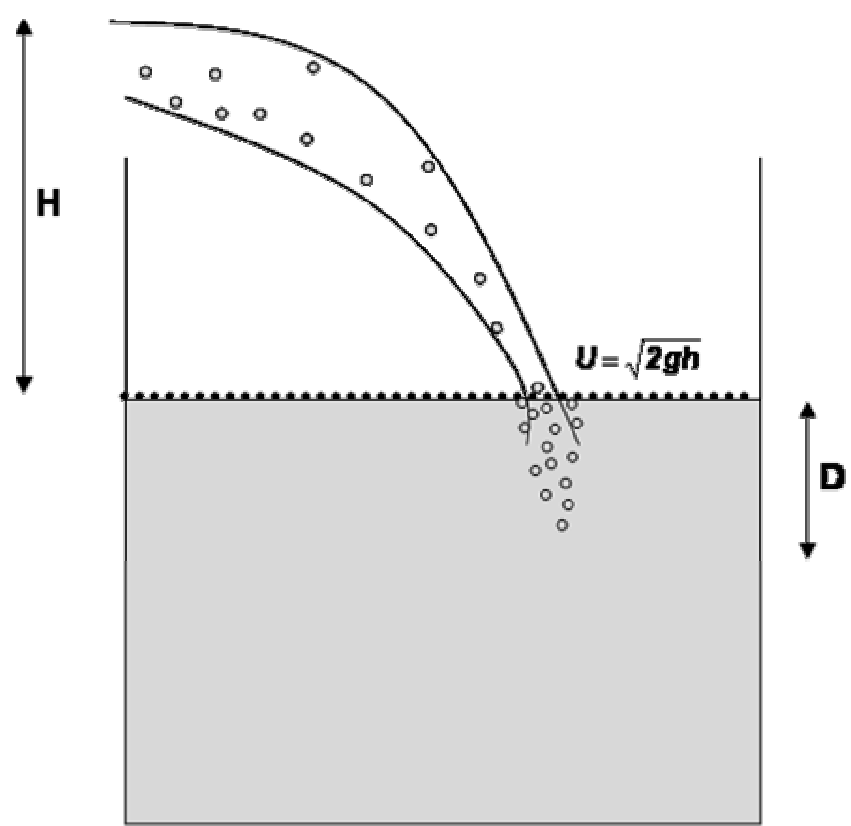

Figure 27. Schematic illustration of metal impingement on liquid metal pool.

The analysis was conducted to determine the depth $\mathrm{D}$ that a reoxidation particle would be expected to travel before its buoyancy caused it to float to the surface of the pool. The force balance is as follows:

$$
\begin{aligned}
& F_{\text {Ladle }}=\rho U^{2} A=\rho_{\text {Particle }} g H\left(\frac{\pi d^{2}}{2}\right) \\
& F_{\text {Gravity }}=m g=\rho_{\text {Particle }}\left(\frac{\pi d^{3}}{6}\right) g \\
& F_{\text {Buoyancy }}=\left(\rho_{\text {Liquid }}-\rho_{\text {Particle }}\right)\left(\frac{\pi d^{3}}{6}\right) g \\
& F_{\text {Drag }}=1 / 2 C_{D} \rho_{\text {Liquid }} U^{2} A=C_{D, \text { Sphere }} \rho_{\text {Liquid }} U_{\text {Liquid }}^{2}\left(\frac{\pi d^{2}}{8}\right) \\
& F_{\text {Static }}=\rho_{L} g D A=\rho_{L} g D\left(\frac{\pi d^{2}}{4}\right) \\
& F_{\text {Ladle }}+F_{\text {Gravity }}-F_{\text {Buoyancy }}-F_{\text {Drag }}-F_{\text {static }}=0
\end{aligned}
$$


Assuming a pouring height of $0.5 \mathrm{~m}$, a particle diameter of $0.001 \mathrm{~m}$ and density of $2100 \mathrm{~kg} / \mathrm{m}^{3}$, and a liquid density of 6900 $\mathrm{kg} / \mathrm{m}^{3}$, the penetration depth is approximately $0.15 \mathrm{~m}$ or about 6 inches. This result suggests that if the metal depth in the sprue is less than six inches, reoxidation particles formed in the sprue cannot float out and will be carried into the runner. The particles may be trapped in the runner if the runner is sufficiently long and flat.[14] However, many steel castings have short runners and high velocities which carry the particles into the mold cavity.

Other studies have examined the metal in the pouring basin from the standpoint of avoiding liquid metal vortexes.[11, 16, 19, 20] In particular, Middleton and Winter used a sprue with a pouring basin having a thin metal plate between the basin and the sprue.[11] The metal plate (0.065 inch aluminum) caused a slight delay in mold filling, during which time a three inch deep metal pool would form in the pouring basin. A similar approach was used in the current study to form a six inch deep pool of metal in the sprue before flow through the runner began.

\subsubsection{Experimental Procedure}

The plate illustrated in Figure 28 was used because of its large cope surface. The flat cope surface facilitated evaluating the reoxidation defects formed.

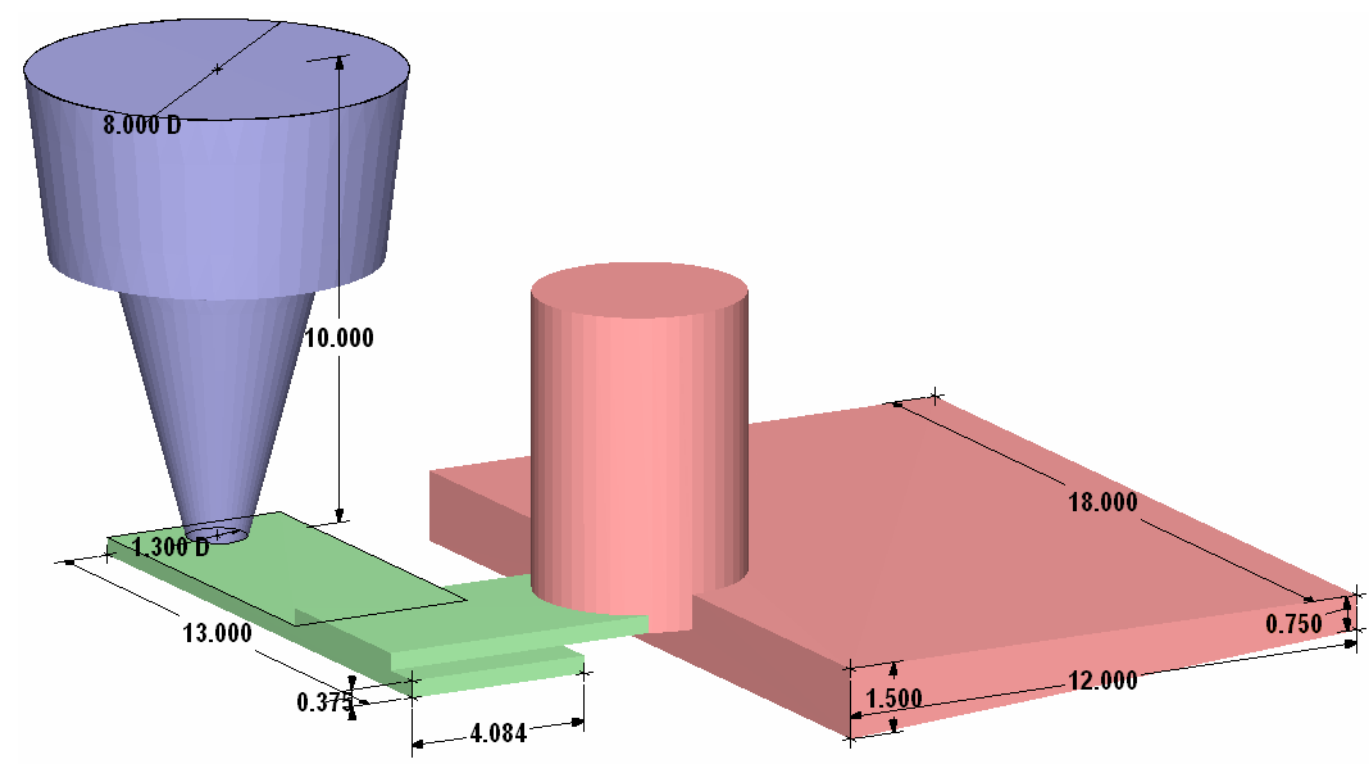

Figure 28. Steel plate geometry with constrained runner and ingate. All dimensions are in inches. 
Two gating systems were analyzed - a traditional 1:2:4 unpressurized system and a constrained system where the flow area remained constant throughout the runner. The effect of liquid pool depth in the sprue was varied by placing a steel "melt-away" plate at the base of the sprue, as illustrated in Figure 29. The sprue volume was also increased so mold filling variations would not cause unexpected variations for the ladle handler.

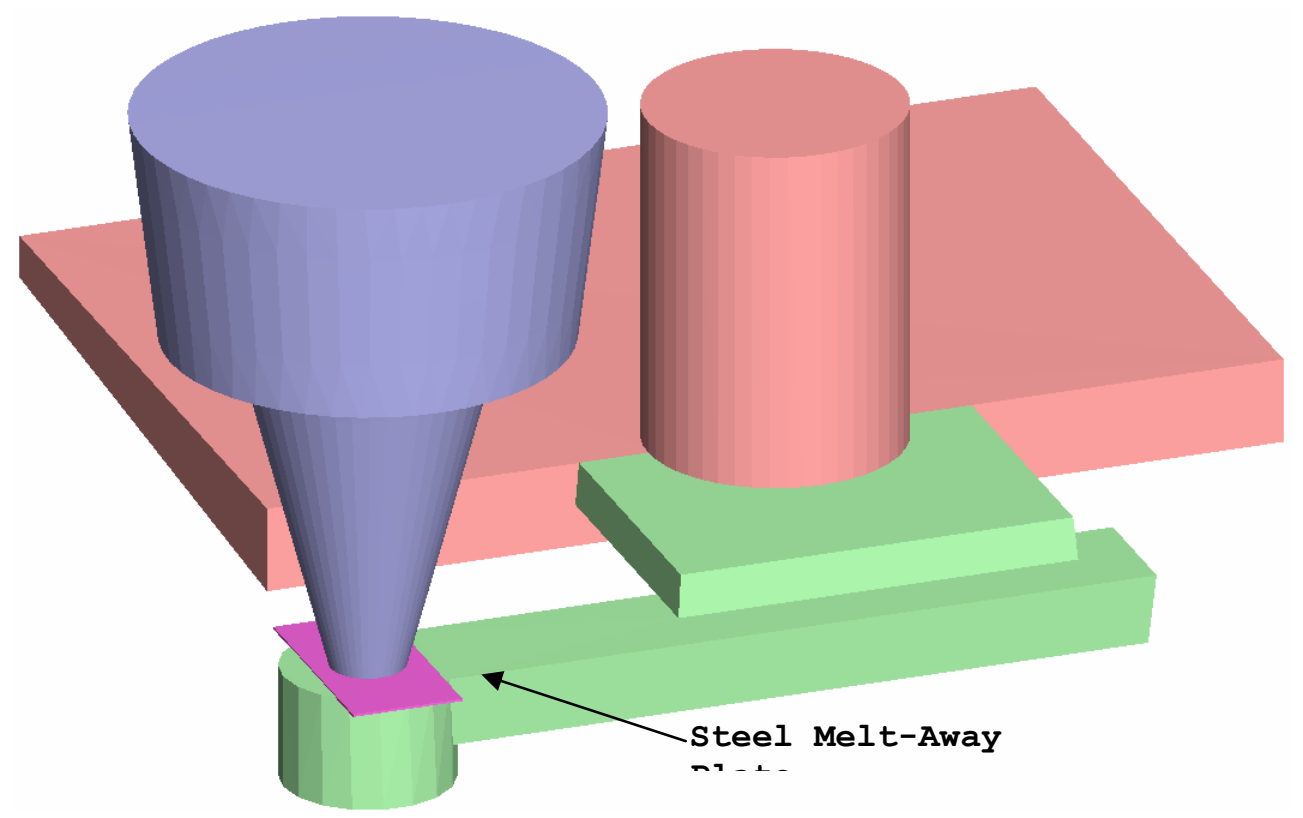

Figure 29. Mold with 0.050 thick steel melt-away plate.

Simulations of both gating systems with and without the melt-away plate were performed and results compared to experimental results. A special subroutine was created to track the formation and movement of reoxidation particles during filling. The subroutine produces a reoxidation particle in each cell having a surface in contact with the atmosphere unless the cell already contains a reoxidation particle.

\subsubsection{Results and Discussion}

$6 \times 9 \times 1.5$ inch plate castings were first poured with a standard sprue and a melt-away plate, blasted, and marked for surface area measurement. Highlighted defects are illustrated in Figure 30. There were no reoxidation defects in castings poured with melt-away plates.

Simulations of the motion of reoxidation particles during mold fill indicated that the melt-away plate allowed the sprue to 
be filled and minimized the entry of reoxidation particles into the casting. Reoxidation particle locations obtained with the constrained gating system with and without the melt-away plate are illustrated in Figures $31(\mathrm{a})$ and (b). The simulation obtained with the melt-away plate retained reoxidation products in the sprue while the casting poured without the plate allowed the defects to enter the casting cavity.

Photographs of the cope surfaces of the plate shown in Figure 29 are illustrated in Figure 32. Castings A and B were poured without the melt-away plate, and Castings $C$ and D were poured with the plate. There were no reoxidation defects on these castings. Reoxidation products were completely eliminated and there was no data scatter. The lack of reoxidation products on the castings poured without a melt-away plate may have been a result of the fact that the large sprue allowed the pourer to quickly fill the sprue and keep it full.

The ladle handlers reported that there was not a noticeable delay when filling molds having the melt-away plate. They also observed that all castings had large liquid metal pool in the sprue during filling, regardless of the presence or absence of the melt-away plate. It was concluded that the large volume of the sprue allowed rapid pool development which resulted in some inclusion "trapping" in the sprue for all castings.

The melt-away plate reduced reoxidation in castings with the smaller sprue and produced cleaner castings. However, the small sprue made it difficult to consistently pour the castings. The large sprue decreases yield, but results in cleaner castings.

\subsubsection{Conclusions}

Gating experiments have been conducted and the results examined using computer simulations. The amount of oxide formed during filling is strongly influenced by the gating system and particularly the sprue.

The liquid surface area integral correlated with casting cleanliness, with higher values of the integral corresponding to more reoxidation defects. Free surface velocity bins better described the liquid free surface during filling. The free surface kinetic was more accurate in trend and magnitude in predicting cleanliness in the coupling hub and the $6 \times 9$ plate.

Simulations have shown that oxidation in the sprue may be responsible for $80 \%$ of the molten metal free surface. Modeling indicated that a 6 inch deep pool of liquid in the sprue might prevent oxide particles from entering the mold cavity. Casting trials conducted with large sprues and melt away plates to produce a deep metal pool in the sprue substantially improved 
casting cleanliness. Carefully controlled confirmation experiments are needed and some consideration given about how to reduce the volume of the sprue and still develop a molten metal pool. 


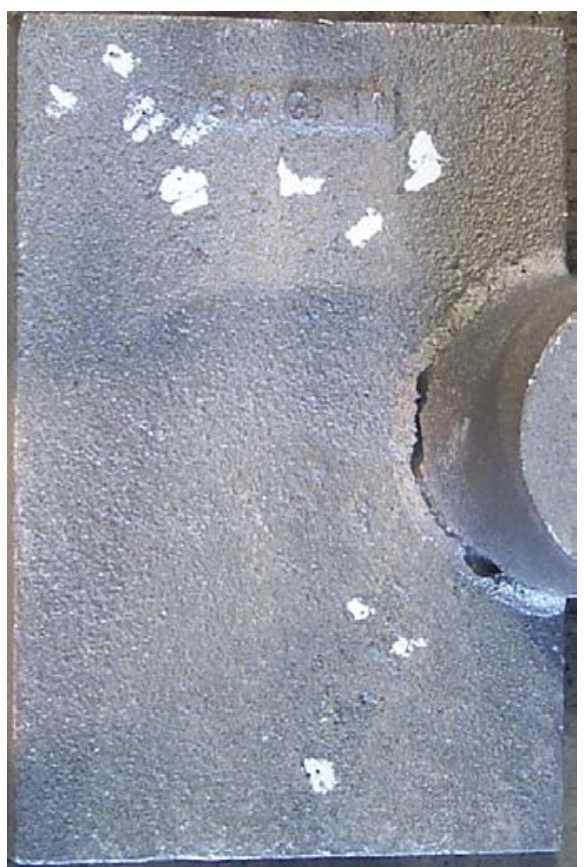

(a)

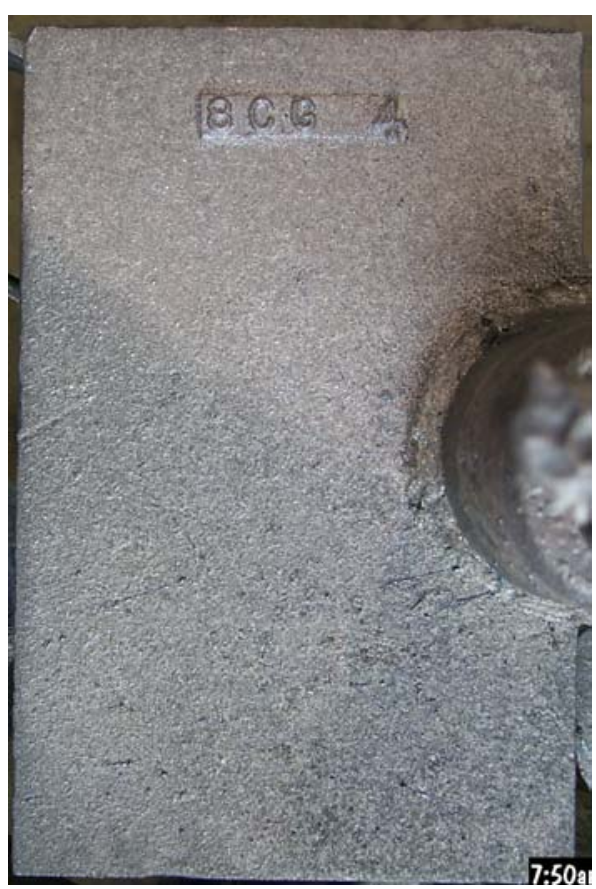

(c)

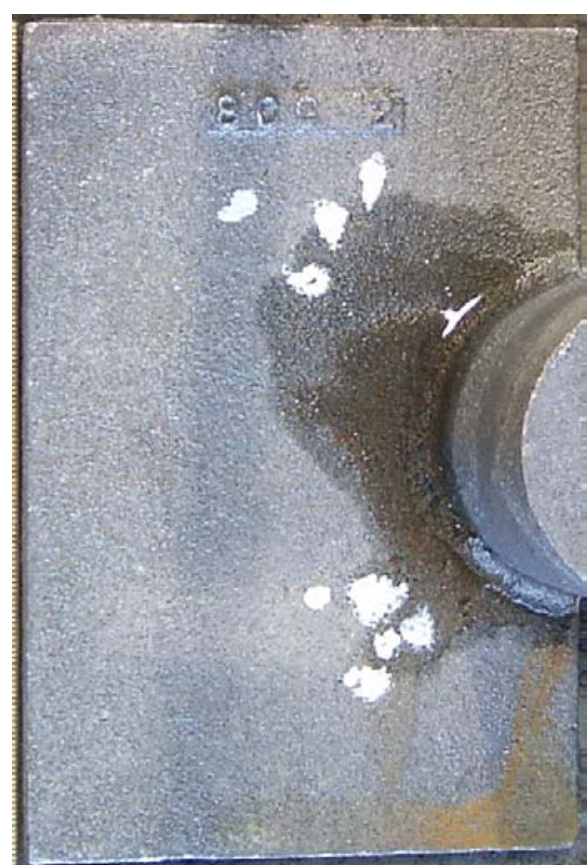

(b)

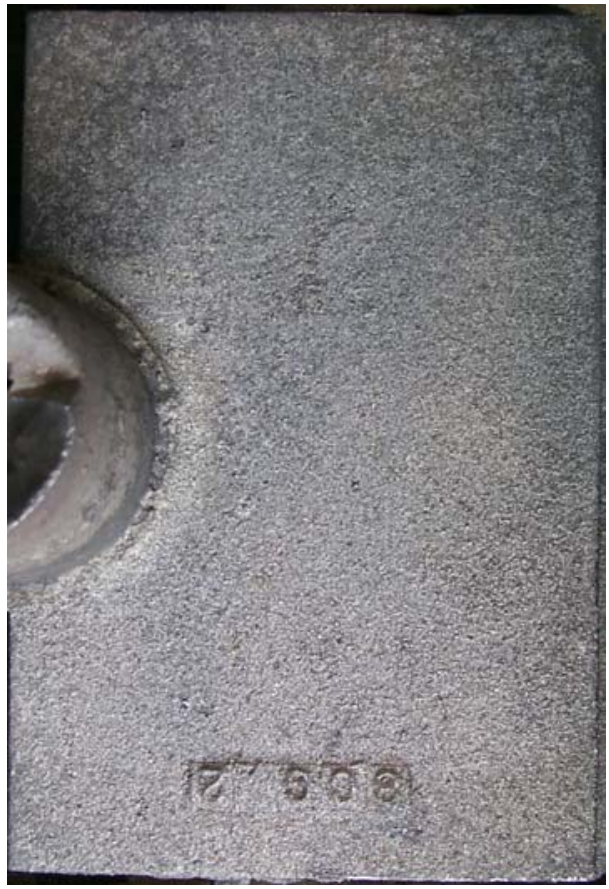

(d)

Figure 30. Cope surface of smaller plate castings used to test the feasibility of the melt-away plate. The images in (a) and (b) were castings without the melt-away plate and (c) and (d) were with the melt-away plate. 


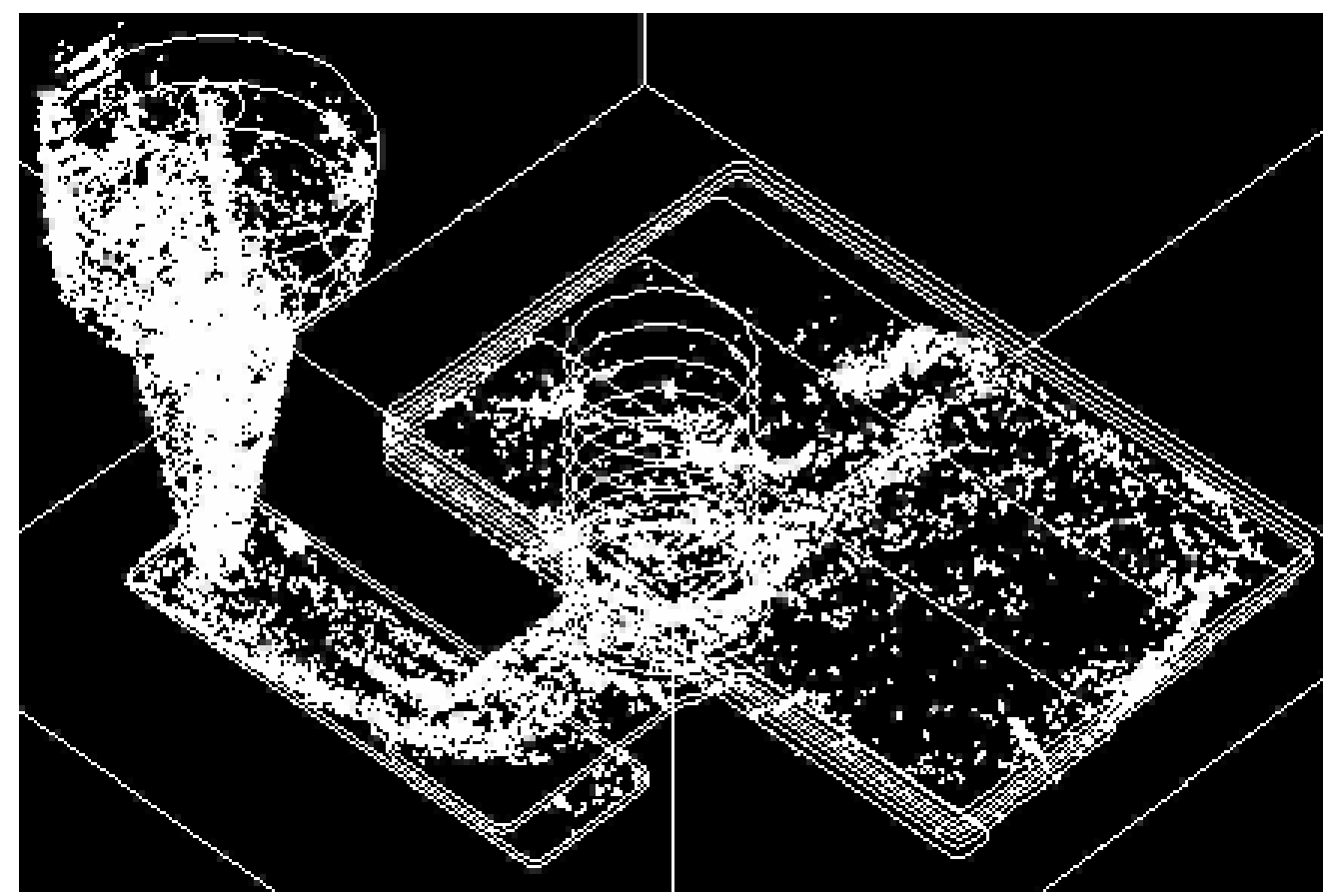

(a)

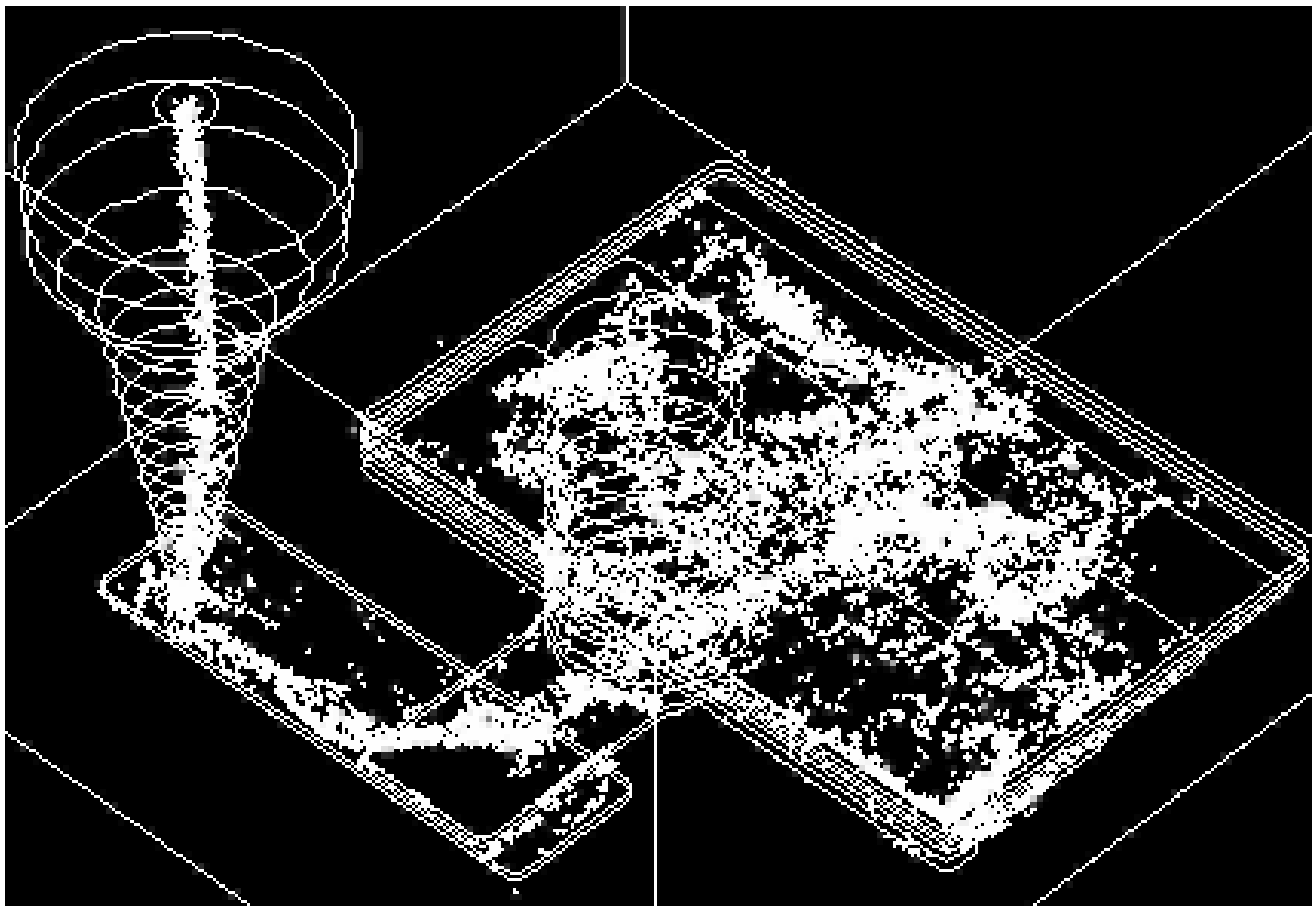

(b)

Figure 31. Simulation results of reoxidation particle location during mold fill (a) with the melt-away plate and (b) without the melt-away plate. 


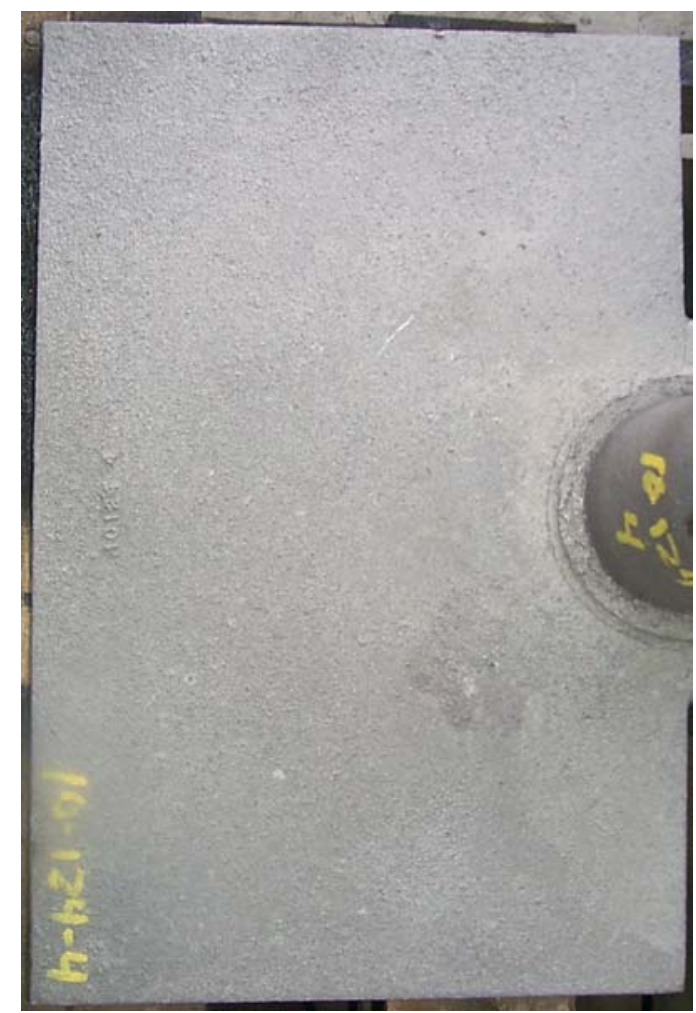

(a)

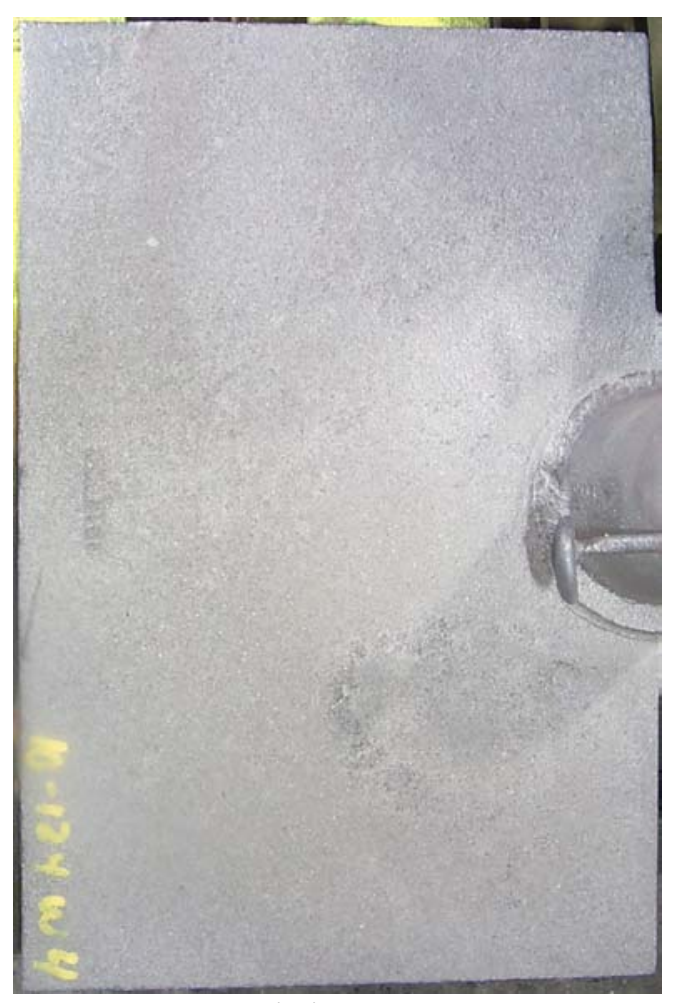

(c)

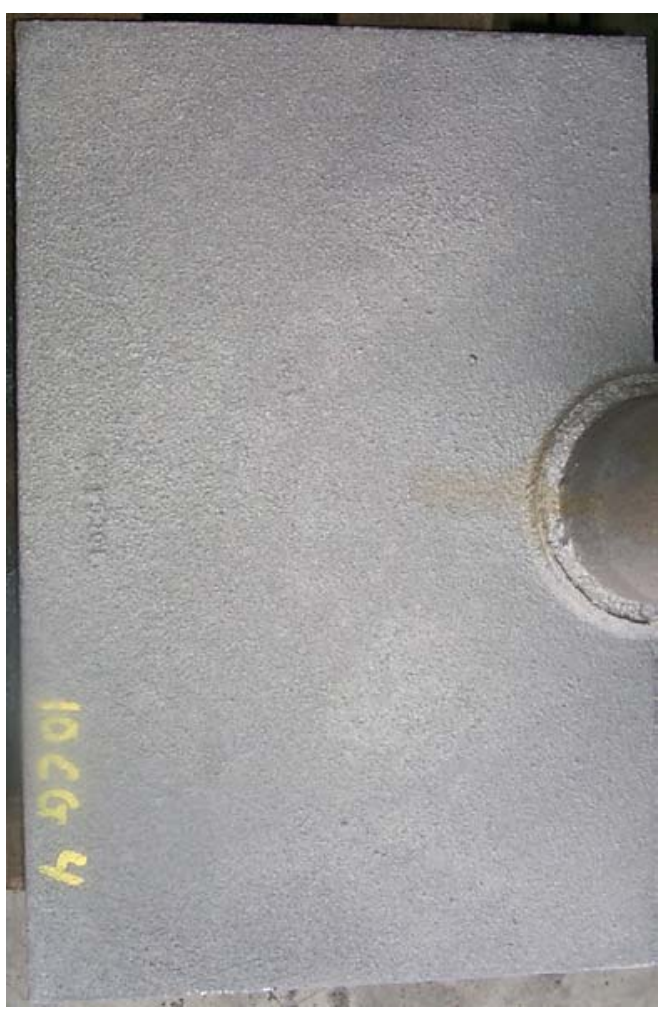

(b)

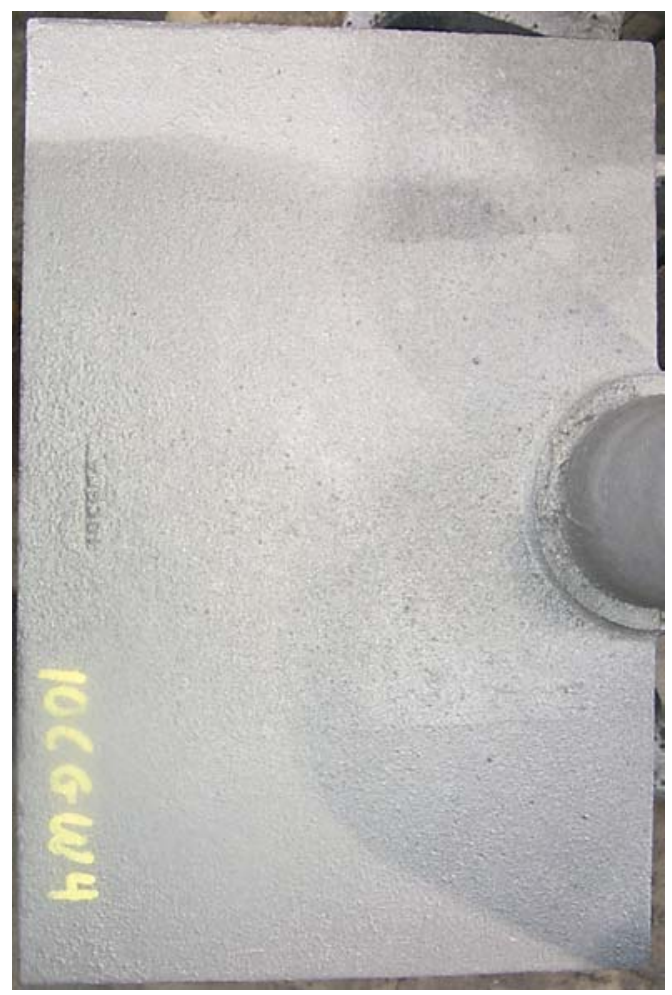

(d)

Figure 32. Cope surface of large plate castings. The images in (c) and (d) are from castings using the melt-away plate. 
CLEAN CAST STEEL TECHNOLOGY, PHASE IV

YEARLY TECHNICAL REPORT

\section{Machinability of Cast Steel.}

Several processing factors affect the machinability of cast steel. The University of Alabama at Birmingham (UAB) has been developing information regarding the influence of foundry parameters on the machinability. The centrifugal casting process adds additional considerations compared to conventional static casting techniques. These include the influence of permanent mold preheat, centrifugal force, and the secondary refining actions during solidification. The need to machine the product is almost intrinsic to the process, and with expanded customer services, it has become increasingly common for machining to be a foundry responsibility.

The centrifugal casting subcommittee of SFSA requested that machinability studies be conducted to address two issues. The development of a meaningful machinability test for centrifugal castings was desired as well as the definition of the magnitude that secondary refining has on machinability. Both factors were considered paramount to advancing machining as another core competency for centrifugal casting foundries. Limited information exists in the literature about the effects of foundry parameters on machinability, and nothing could be found to relate machinability of centrifugal castings to results obtained with static castings. There was a need for comparative data.

An estimate of the percentage of centrifugally cast steel that is machined is illustrated in Figure 33. Almost all the inner diameters and ends of these castings are machined and machining is necessary for a significant fraction of the outer diameter of the castings. The cost impact of machining relative to other foundry core competencies is illustrated in Figure 34. For vertical castings, machining costs can contribute around 50\% of the total cost to produce the castings. The percentage drops to about 20\% for machining of horizontal castings but remains one of the highest cost contributors. These numbers indicate that the SFSA machinability work can have a significant impact on profitability in this industry. Far more attention has been placed on the casting side of operational costs without considering the relationships to the cost impact on the machining side of the process. 


\section{Percentage of Product Machined}

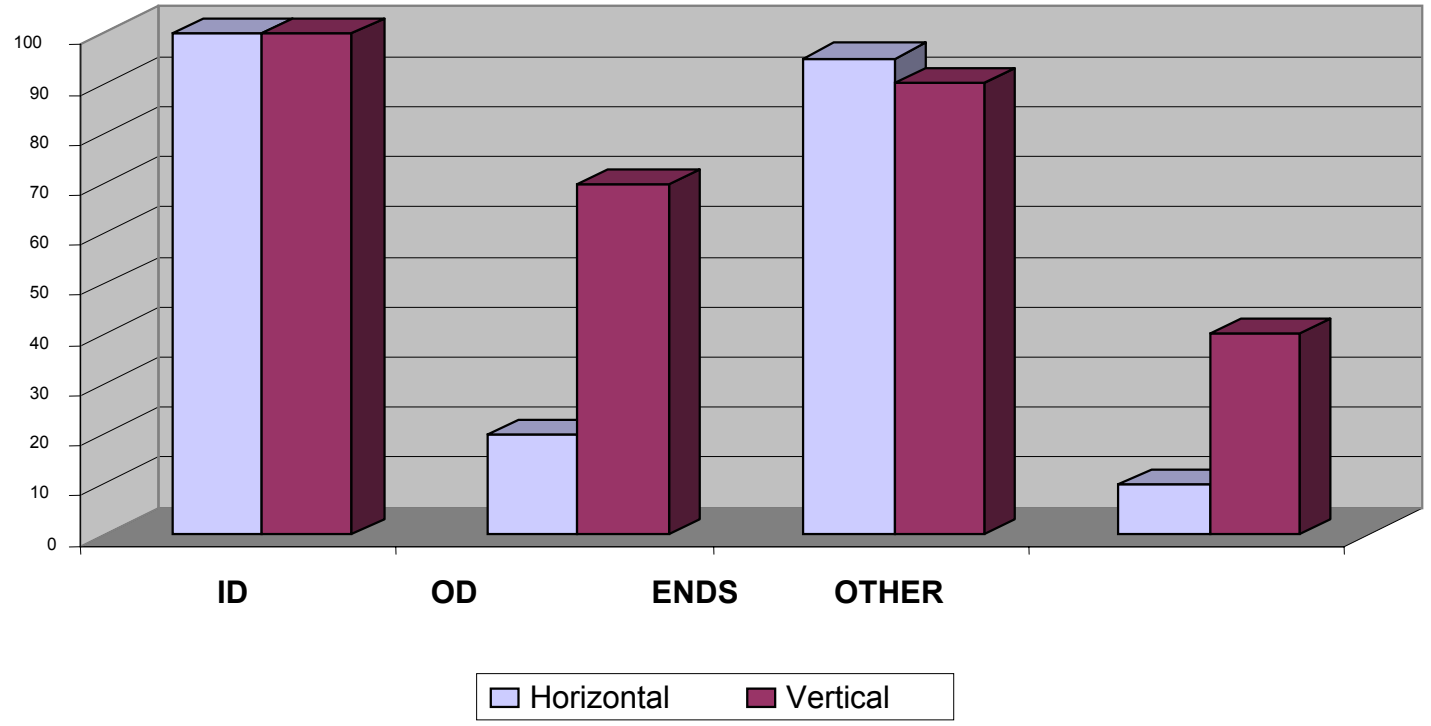

Figure 33. Estimated Percent of Centrifugally Cast Steel that is Machined.

\section{Pareto of Cost Factors ( $\%$ of Total )}

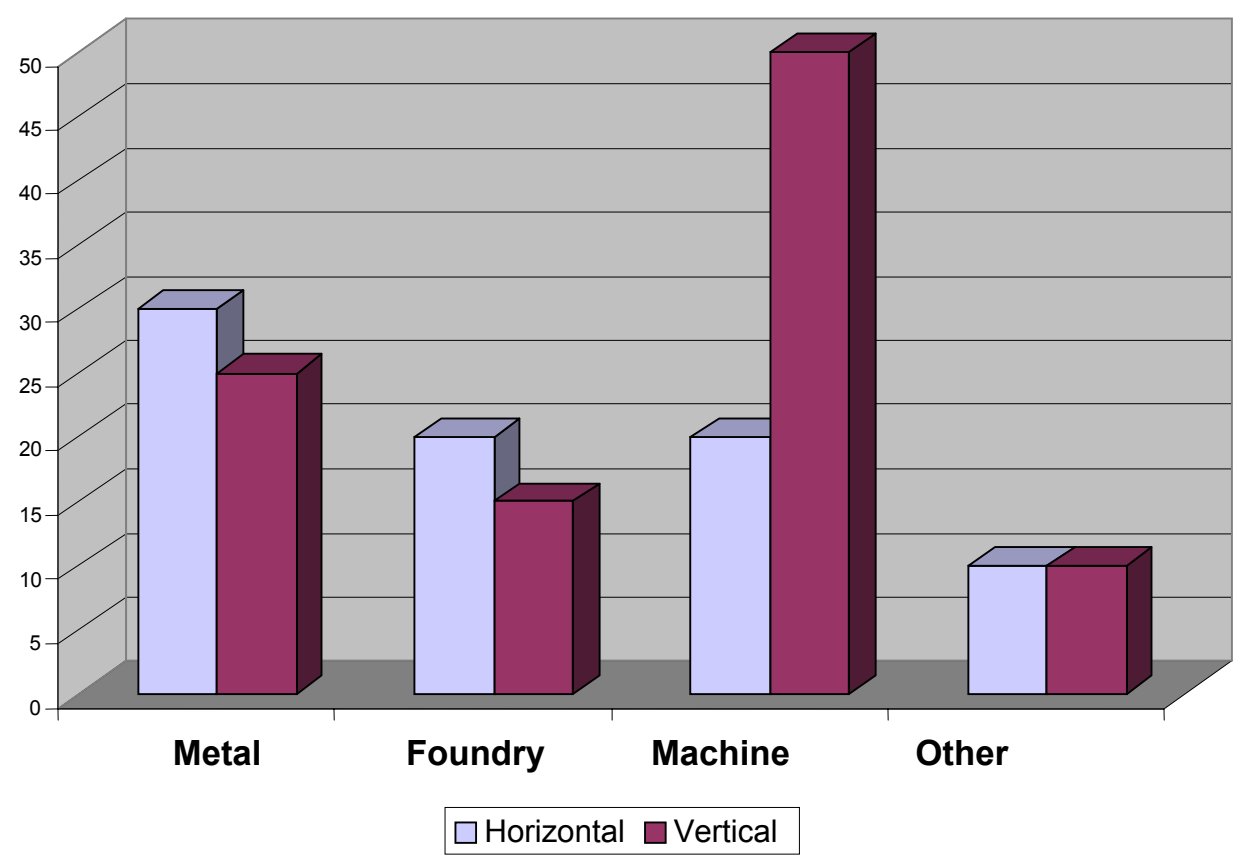

Figure 34. Cost Impact of Machining of Centrifugally Cast Steel Relative to Other Factors. 
It was found in earlier studies that micro-inclusions had significant impact on tool life and metal removal rate. Reaction products formed during liquid metal treatment and processing produce inclusions with hardnesses ranging from 2 to 13 times that of the steel matrix, as shown by data presented at the 1999 SFSA $T$ and O Conference. (22) This data provides some insight into the range and hardness of abrasive particles that might be introduced to steel during deoxidation and re-oxidation formed during steel processing. Most foundries employ a number of furnace or ladle treatments that produce some of these oxides.

Centrifugal casting provides one feature that varies substantially from most other casting processes. For any given cast wall and shape, centrifugal castings usually have a finer grain structure due to the large heat sink provided by the permanent mold and the open ID cooling. Thin walled horizontal centrifugal castings often have a columnar macrostructure at the casting $O D$ with a sharp transition to a fine grain equiaxed structure toward the ID. These changes in structure occur during significant shifts in solidification dynamics affected by complex environmental conditions.

Both the grain structure and deoxidation practice have been found to have a significant effect on machinability of centrifugal castings. The relevance to centrifugal castings of the prior work done on static sand castings was questioned because of differences in grain size and the secondary refining unique to the centrifugal process.

A machinability study was needed that would determine the effects of processing and grain size in centrifugal castings. A suitable study would need to:

a) Consider changes in inclusion distribution across the refined zone,

b) Be insensitive to phase distribution influences,

c) Be based on a machining method more relevant to centrifugal castings,

d) Measure the effects of casting grain size, and

e) Test the observations of earlier works relative to the effects of inclusion type and size.

The solution chosen was to use Taguchi DoE methods to determine the main influences, minimize the number of tests to be performed, and develop machinability procedures adaptable to centrifugally cast tubes.

\subsection{TUBE MANUFACTURE}

The material examined in this study was a common heat resistant stainless steel. This material, as produced for this study, has a stable fully austenitic structure with 
interdendritic carbides and is free of minor phases such as ferrite. It requires no heat treatment, and this eliminates one source of machinability variation. The Taguchi matrix was used to evaluate the effects of extremes in commercial production conditions.

One standard mold coating method and deposit thickness was applied in the production of all castings. Eight trial castings were produced from charges made from one lot of AOD processed ingot. Only the furnace and ladle treatments and certain pouring parameters were varied. The ends of the tubes were discarded to eliminate end effects. Half of the remaining material was used in machining experiments at $\mathrm{UAB}$, and half was tested at Metaltek for conformance to physical and chemical requirements. The material was also examined for grain distribution and dendrite size to confirm the structures actually produced.

Seven foundry parameters or factors were varied. Each of the factors was tested at two test settings. The variables included the total weight of furnace and ladle deoxidation additions, the type of addition made in the furnace, the type of addition made in the ladle, the pouring temperature, mold temperature, centrifugal force, and interactions between total additions and centrifugal force. The total weight of furnace and ladle deoxidations was varied from 2.5 to 1 lbs/1000 lbs metal. The type of additions made at the furnace was CaSi or Al and at the furnace were CaSi or $\mathrm{NiMg}$. The pouring temperature was varied between 2600 and $2900^{\circ} \mathrm{F}$, the die temperature was varied between 350 and $500^{\circ} \mathrm{F}$, and the centrifugal force was varied between 90 and $120 \mathrm{G}$.

\subsection{MACHINABILITY PROCEDURES}

Three-quarter inch square, uncoated fine-grained carbide (SPG 631) inserts were used to perform the tool life (wear) experiments. The inserts were 0.1900 inches thick with an 18 degree taper. Each insert was examined for cutting edge uniformity before use. The presence of a chipped edge or other anomalous feature caused the insert to be discarded. The insert was placed on a gauge block with parallel sides such that three of the corners overhung the gauge block and the fourth corner overhung a center hole on the block. This prevented any edge build-up on a cutting edge from affecting the wear pattern determination.

The flank of the tool showed progressively more wear as more metal was machined from each casting. The area and shape of the wear land on each insert edge was recorded periodically by removing the tool from the CNC mill and digitally recording the wear pattern. The rate of flank wear was related to the machinability of the material by plotting the average wear as a 
function of the volume of material removed.

Each insert was used until it had an average wear of 0.015 inches across the cutting edge or until it had been used to remove at least 70 cubic inches of metal. The insert wear rates obtained with each material were determined using previously described procedures (23).

Turning experiments using face cuts were performed at 550, 700,850 and 1000 surface feet per minute (sfm) on each material, and triplicate experiments were performed at each speed. All experiments were performed at a feed per revolution of 0.030 inch. Statistical analysis was performed on the data obtained at the two lowest speeds because wear data at lower speeds reflects the abrasiveness of the phases in the metal while minimizing the possibility of tool edge overheat often encountered at higher speeds.

A schematic illustration of a worn insert is shown in Figure 35. Typical flank wear areas, measured as metal was removed from a casting, are illustrated in Figure 36. The flank area divided by the flank wear length provided the "average" flank wear.

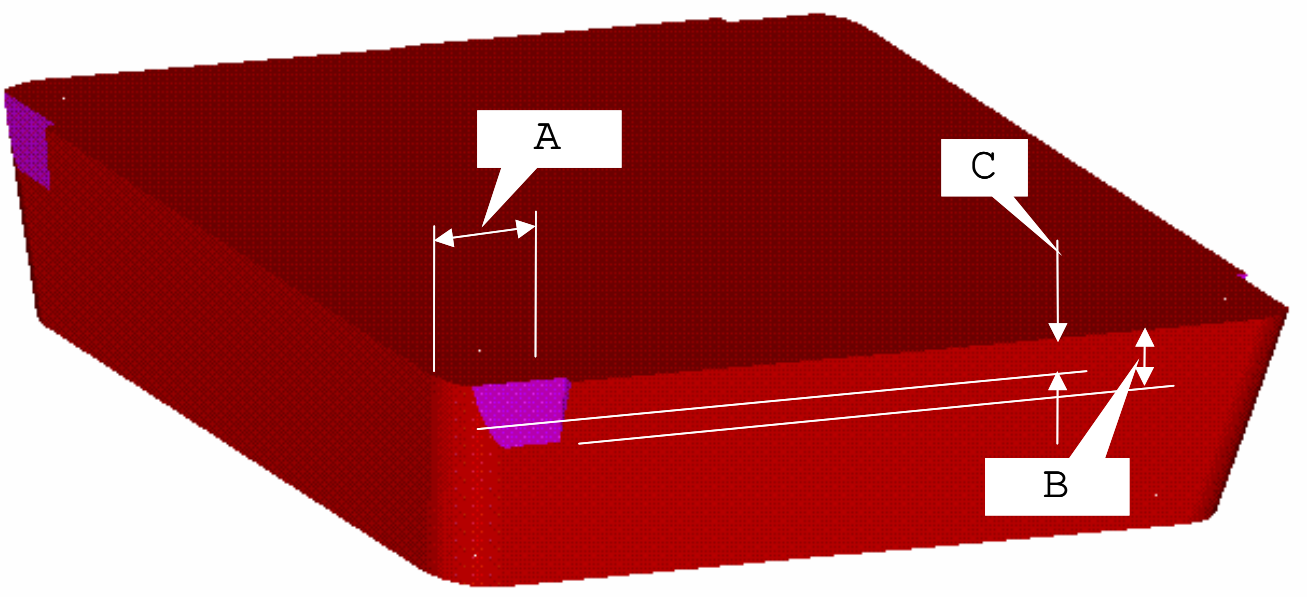

Figure 35. Schematic Illustration of Worn Insert. A = Depth of Cut, $\mathrm{B}=$ Maximum Wear Height, $\mathrm{C}=$ Average Wear. 


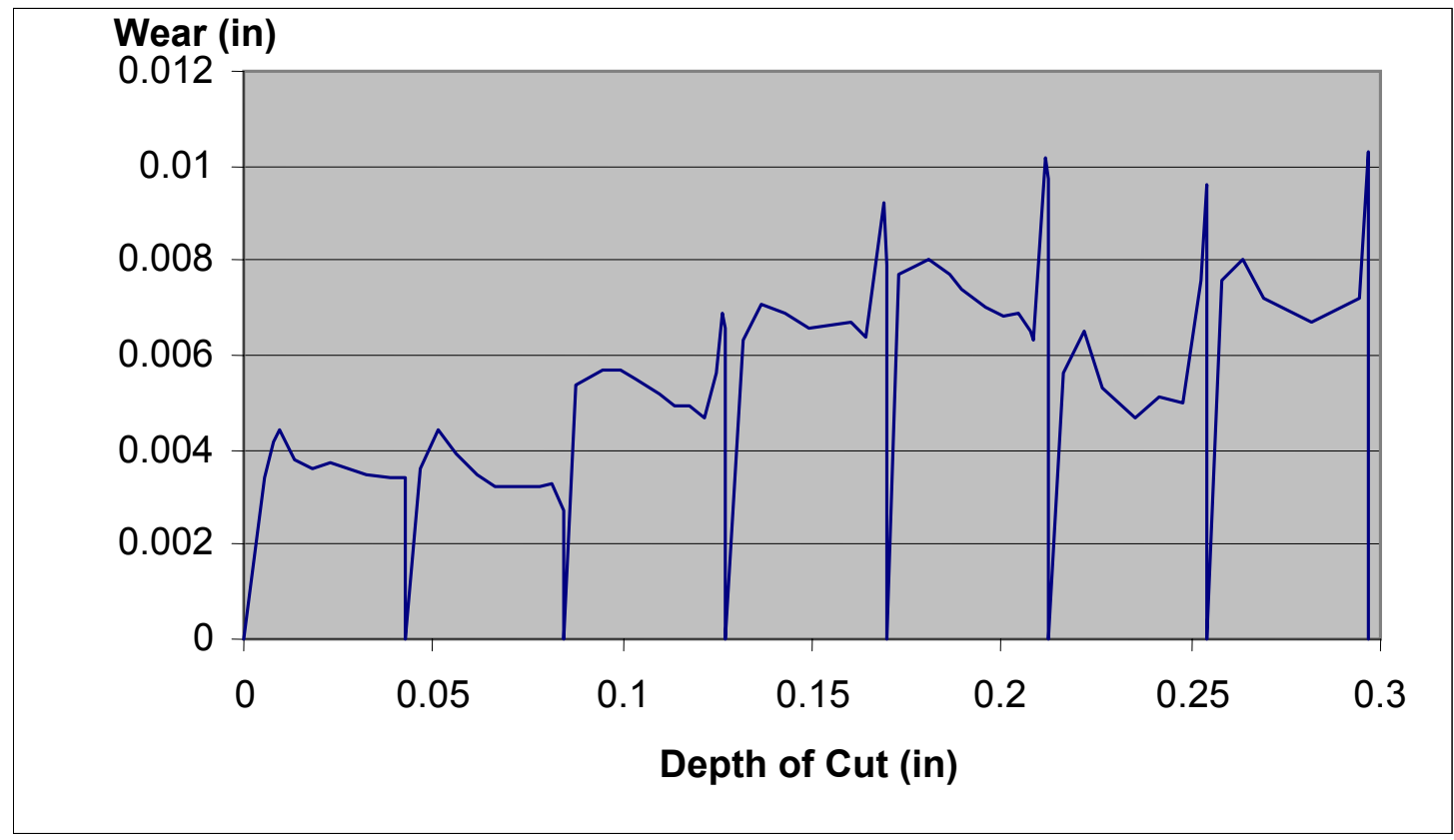

Figure 36. Example of Progressive Tool Wear on Machining Tool.

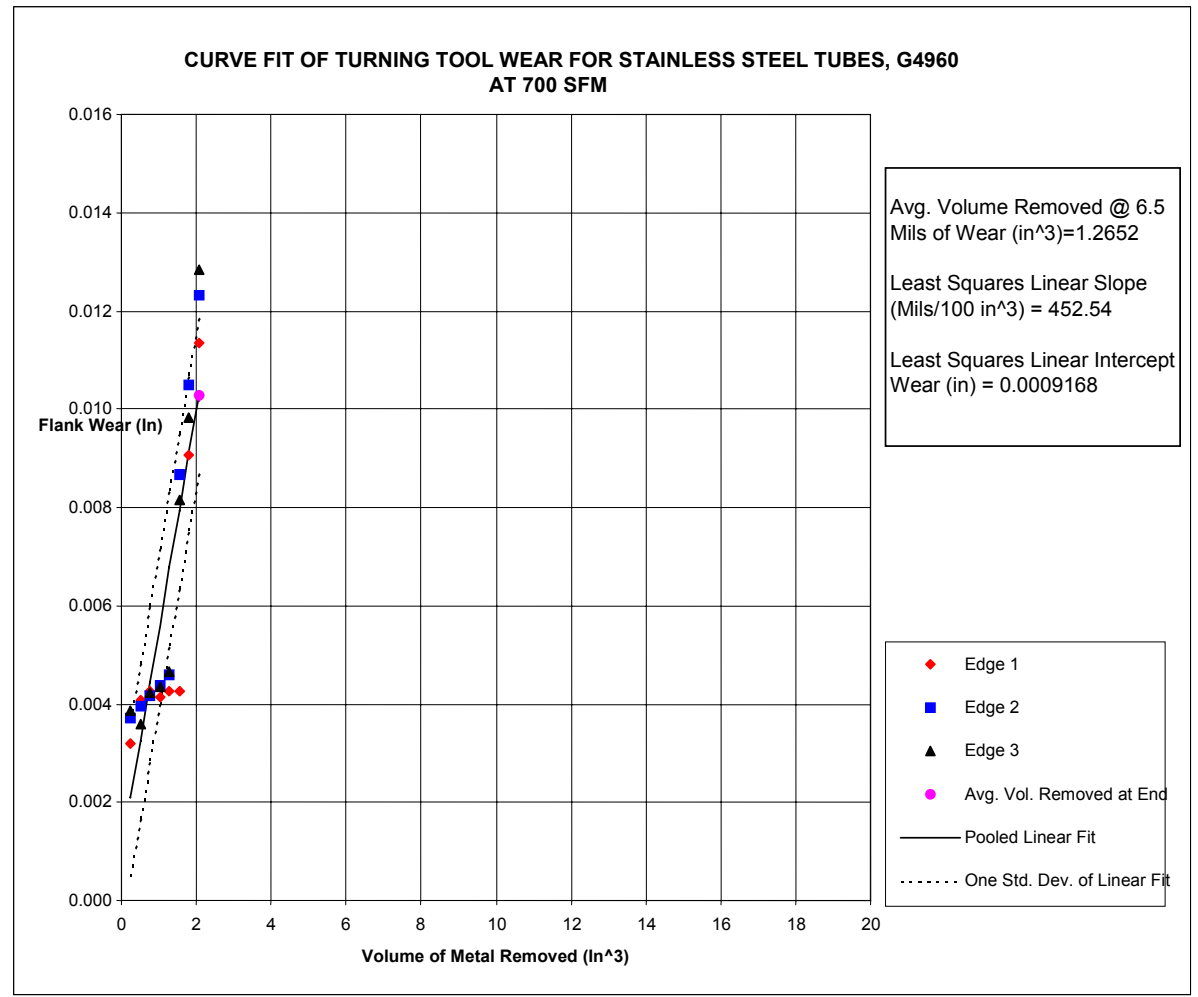

Figure 37. Representative Tool Wear from Stainless Steel Tubes. 
A typical tool wear curve obtained with triplicate data is illustrated in Figure 37. The solid line drawn through each data set represents the best linear fit to the insert wear data, and the dashed lines on either side of the solid line represent one standard deviation in tool wear. Tool wear data obtained under specific conditions is summarized in the boxes on each of the graphs. The data include the volume of metal machined away before the tool reached $6.5 \mathrm{mils}$ of average wear, and the least squares linear slope of the wear curve. The data obtained with each insert edge was plotted with a different symbol so the wear on any insert can be followed if desired.

The tool wear curves generally show some initial "break-in" wear followed by a steady-state wear region. The initial wear occurred as the tool tip was abraded and perhaps chipped. The approximate linear wear region was a function of the cutting speed and the metallurgical characteristics of the material being machined.

\subsection{STATISTICAL ANALYSIS OF MACHINABILITY DATA}

Design of Experiment (DoE) is a statistical technique introduced in the 1920's by R.A. Fisher in England. Although DoE attracted much development in the academic environment, it remained too complex a tool for everyday use in the manufacturing industries. In 1940, Dr. Taguchi began developing a more userfriendly technique. His goal was to harness a powerful mathematical tool that could shorten development cycles and improve the "bottom line" in his manufacturing operation. The Taguchi DoE was introduced in the United States in the 1980's using a blend of traditional DoE methods and assumptions made by Taguchi.

The methodology was further simplified with advances in computer software in the 1990's. Today the Taguchi approach and other DoE methods are used in many industries as an economical means of analyzing product/process design and optimization projects.

System interactions are very complex in most foundry studies. Seldom can a process be fully explained by simple XY relationships. Metaltek began using Taguchi methods in about 1996 and has saved many hours in finding developmental path approaches to complex issues. It seemed a natural application for the SFSA-DOE project on machinability of centrifugally cast products.

The goals for this study were to:

a) Determine if a lathe machining procedure would be sensitive to processing changes in the centrifugal casting process, 
b) Determine if the centrifugal secondary refining process completely outweighed the effects of deoxidation practice and conduct the experiments with the fewest tests possible

c) Rank the relative importance of major process variables on machinability

Prior to the development of DoE techniques, many of us were schooled in the experimentation belief that "you can only vary 1 thing at a time." However, from production observations, we believed that most machinability variations could be caused by several variables, and judicious selection of the process variables was used in this DoE study. The classic full factorial experiment would require producing and evaluating 128 test combinations. The partial factorial Taguchi approach focused on seven variables that required the production of eight batches of steel. This approach also provides a weighed ranking of the importance of each factor and a measure of the scientific "noise" in the study.

Machinability was measured several ways before reaching the final procedure. In all the initial test cases, the ANOVA error factor indicated serious flaws in the methodology. The following results are based on the final method

\subsection{TOOL WEAR DATA}

Triplicate tests were performed for each heat at the four speeds tested and the results from all three tools are included. As would be expected, the linear wear slope increased and the volume removed at $6.5 \mathrm{mils}$ of wear decreased with higher turning speeds. Tool wear rates generally increase at higher turning speeds because tool heating increases.

Statistical analysis on the machinability data was performed on both the linear wear and the volume removed data. The higher surface speed data (850 and $1000 \mathrm{sfm}$ ) were not used in the statistical analysis due to overriding effect of frictional heating on tool wear.

A strong contributor to the variation in machinability for both volume removed and linear wear rates was turning speed. Higher turning speeds gave increasing wear rates and decreasing volume of material that was removed at $6.5 \mathrm{mils}$ of tool wear as would be expected.

The furnace additions were the only variables that had a statistically significant contribution (at the 95\% confidence level) on the variance in the machinability as measured both by linear wear slope and volume of material removed at $6.5 \mathrm{mils}$ of wear. Better machinability (lower wear slopes and higher volume removed) was achieved with CaSi additions than with Al additions. 
These additions also had the highest or close to the highest percent contribution to the variation in the machinability of all the foundry changes made.

The statistical significance of machinability variation from the add sum, ladle additions, pouring temperature, and die temperature results was not consistent between the linear slope and the volume removed measurements. The linear wear results indicated that ladle additions of NiMg gave better machinability than CaSi while the volume removed showed no contribution. The volume removed measurement showed a statistically significant contribution from the add sum, the pouring temperature and the die temperature. These measurements indicated that a higher add sum, a higher pouring temperature, and a higher die temperature correlated with lower wear rates. The linear wear results showed no correlation with the variation in these conditions.

The centrifugal force did not show a statistically significant contribution to the variance in machinability.

\subsection{MICROSTRUCTURAL ANALYSIS}

Representative through thickness samples of the casting were removed, polished, etched with Vilella's Reagant and examined with an optical microscope. A typical microstructure of the steel investigated in this study is illustrated in Figure 38 at $110 \mathrm{x}$ and 550x. The microstructure was austenitic with interdendritic carbides. Small inclusions were also present in the structure as shown in Figure 38.

(A)

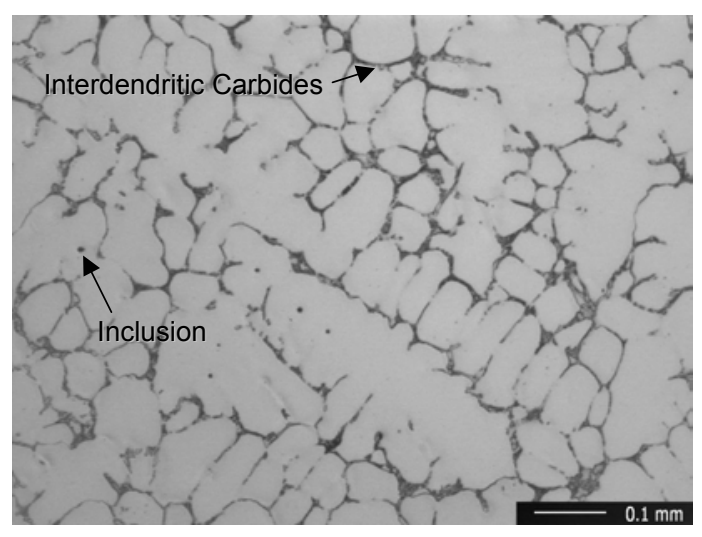

(B)

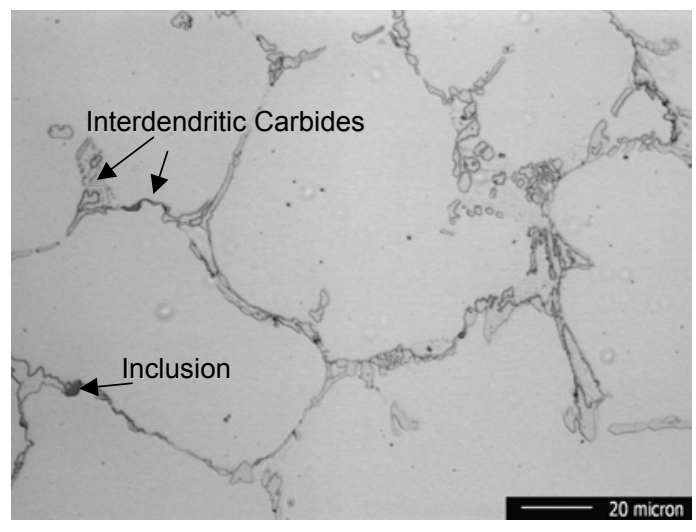

Figure 38. Representative Microstructure (Trial 1). Villella's Reagant. (A) 110X. (B) $550 \mathrm{X}$.

Average dendrite arm spacing (DAS) was measured on the samples. 
The dendrite arm spacing varied from 0.0154 to 0.02311 inches. Regression analysis between the machinability results at $550 \mathrm{sfm}$ and the microstructural parameters was performed using linear and exponential fits. A turning speed of $550 \mathrm{sfm}$ was chosen because thermal effects due to tool heating should be the lowest at this speed. This examination showed a statistically significant but relatively weak correlation between lower dendrite arm spacing and decreased wear rates. These results are consistent with increased machinability seen with increased pouring temperatures as increasing pouring temperatures lead to lower dendrite arm spacings.

Macroexamination of the grain structure was also performed and the percent of the wall thickness that had a columnar grain structure was measured. The percent wall thickness with columnar grains ranged from 0 to $80 \%$. No correlation between the machinability at $550 \mathrm{sfm}$ and the percentage of columnar grains was found.

The samples were also examined in a scanning electron microscope (SEM) equipped with energy dispersive x-ray spectroscopy (EDS). A typical backscatter electron image from the SEM is shown in Figure 39 at a magnification of 425X. Contrast in backscatter electron images arises primarily from compositional differences. Phases with a higher average atomic weight are brighter. Two different carbides were evident from this examination. The darker carbide was rich in chromium, and the lighter carbide was rich in niobium.

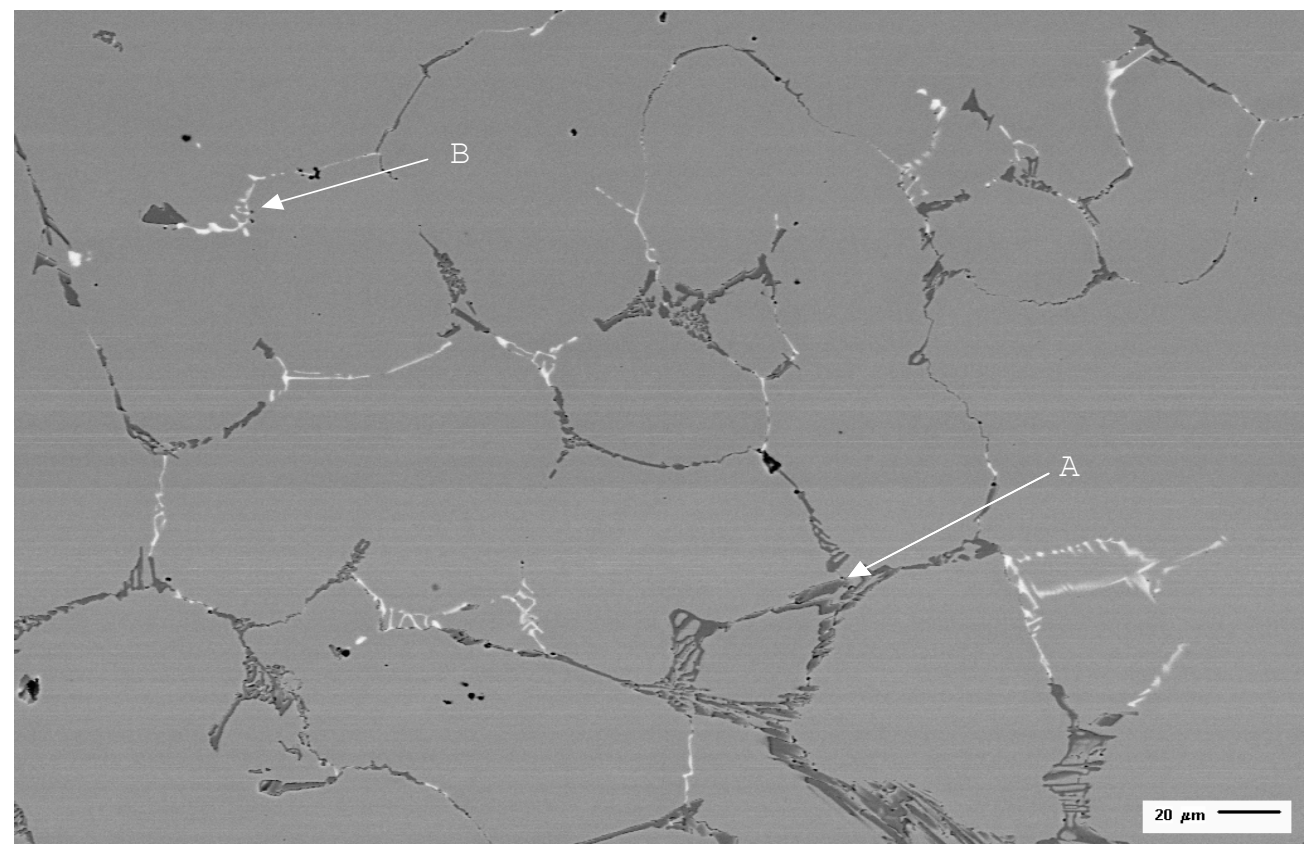

Figure 39. - Representative Back Scatter SEM Image of the Microstructure. 
The volume fraction of each type of carbide was measured using automatic image analysis. Thirty backscatter SEM images were acquired from each material at a magnification of 500x. The volume fraction of chromium carbide ranged from about 3-4\% and the volume fraction of niobium carbide ranged from 1-1.4\%. The total carbide level ranged from 4.2 to $5.4 \%$. A statistically significant trend of increased wear rates with increasing volume percent carbide was seen. However, no firm conclusions regarding these results can be made because the range in the carbide level was so low.

The volume percent of the interdendritic regions was also measured. This area includes both the austenite and the carbides that lay between the austenitic dendrite arms. A moderate correlation $\left(\mathrm{R}^{2}=55 \%\right)$ of a decreased wear rate with increasing volume percent of interdendritic regions was found.

The inclusions in each sample were analyzed using an SEM. Inclusion count data was obtained by examining 30 fields at a magnification of 500x. Every inclusion was identified using the EDS system. The size and type of inclusion was rated, and the number per area and approximate volume percent calculated. All inclusions identified contained oxygen. Some inclusions were rich in aluminum and others were rich in silicon. Lesser amounts of magnesium and calcium were also found in the inclusions.

Analysis of the data indicates that there was a statistically significant association (>95\% confidence level) between the type of inclusion that formed and the furnace addition. Only Si-rich inclusions were found in the castings with furnace additions of CaSi, while mostly Al-rich inclusions formed in the castings with furnace additions of aluminum. The only foundry change made that had a statistically significant effect (at the 95\% confidence level) on the variability in the machinability as measured both by the linear wear slope and the volume of material removed at 6.5 mils of wear was the furnace additions. The type of inclusion formed may be contributing to the change in the machinability as $\mathrm{Al}_{2} \mathrm{O}_{3}$ is significantly harder than $\mathrm{SiO}_{2}$.

\section{6 MECHANCIAL PROPERTY MEASUREMENTS}

Two samples were removed from each heat for tensile property measurements. The yield and tensile ranged from 37-43 ksi and from 64-73 ksi, respectively. No correlation between these properties and machinability was found. The narrow range of tensile property values precludes any correlation.

Brinell hardness measurements were performed using a $500 \mathrm{~kg}$ load at the casting midwall. Thirty measurements were made per material. The hardness ranged from 130-139 BHN. There was no 
correlation between the machinability results and the Brinell hardness. Similar to the tensile properties, the narrow range of hardness values probably explains this lack of correlation.

The microhardness of the austenite dendrites was measured using a Vickers indenter with a $50 \mathrm{gm} \mathrm{load.} \mathrm{Twenty} \mathrm{indents} \mathrm{were}$ made per material, images of the indents were recorded with an optical microscope at 500x and measured using an image analysis system. The microhardness of the austenite ranged from 182 to 215 VHN. There was not a statistically significant correlation between the austenite microhardness and the machinability of the castings.

\subsection{CONCLUSIONS}

From the foundry executive's perspective, these eight test castings have shown that:

1) A machinability test sensitive to centrifugal casting parameters was developed.

2) This test based on turning cuts at 550 SFM is more meaningful to centrifugal castings than drilling based tests on flat product.

3) Potential exists to further enhance the test's relevance by using lower SFM used for production work.

4) Centrifugal castings machined with lower tool wear rates than previously tested static castings.

5) Findings were consistent with the earlier work conducted by UAB. Machinability was better in the castings produced with furnace additions of CaSi than with those produced with furnace additions of Al. Furnace additions that lead to the formation of harder aluminum oxides should be avoided.

6) Lowering the machining cost of our product seems possible.

7) Ladle additions of NiMg instead of CaSi may have given better machinability results although the results were inconclusive. This was most probable because of its scavenging or oxide flotation capacity.

8) The higher G's at which the dies were run did not help machinability.

9) Machinability may be increased with careful control of the microstructure. There were indications that 
machinability increased with smaller dendrite arm spacing, lower volume percent of carbide and higher volume percents of inter-dendritic regions. 


\section{References}

1. M. Blair and R. Shepherd, "The Effect of the Smoothflow/CTA Cruciform Nozzle on the Incidence of Oxide Macroinclusion Formation", Steel Founders' Society of America Technical \& Operating Conference, Chicago, IL, pp. 12 (November 14-16, 1991).

2. J.M. Svoboda, R.W. Monroe and G. Vingas, "Laminar Flow Bottom Pour Nozzle Development", Electric Furnace Conference Proceedings, p 251-258 (1986).

3. Steel Founders' Society of America, Clean Cast Steel Technology - Research Report No. 6, January 1994.

4. John D. Carpenter and R. G. Shepherd, "Clean Steel Project Identification of Variables That Affect Cope Oxide Inclusions In Steel Castings", Steel Founders' Society of America T\&O

Conference, November 1996.

5. R. D. Pehlke, Unit Processes of Extractive Metallurgy,

Elsvier North Holland, New York, 1982, p 44-45.

6. W. C. Leslie, Personal Communication, October 1976.

7. B. K. D. P. Rao and D. R. Gaskell, Metallurgical

Transactions B, V12 B, p 311-317, June 1981.

8. R. D. Pehlke, Unit Processes of Extractive Metallurgy,

Elsvier North Holland, New York, 1982, p 218.

9. E. J. Turkdogan, Fundamentals of Steelmaking, Institute of Materials, London, U. K., 1996, p 98.

10. SFSA Report: March 1993, pp. 1-11.

11. J.M. Middleton and R.M. Winter, Journal BSCRA, No. 95, March 1967 .

12. M.C. Ashton, Journal of Research, SCRATA, No. 47, December 1979.

13. G. Hartay, 1998 SFSA Technical \& Operating Conference.

14. W.T. Adams, K.W. Murphy, and C.D. Ricks, 1999 SFSA Technical \& Operating Conference.

15. L.W. Stuart and F.W. Boulger, SFSA Research Report, No. 52, July 1962 . 
16. X. Yang and J. Campbell, International Journal of Cast

Metals Research, 1998, No. 10, pp. 239-253.

17. P. Scarber, Jr., 2000 SFSA Technical \& Operating Conference.

18. B. Hanquist and J. Carpenter, 2000 SFSA Technical \&

Operating Conference.

19. D. Smith and T. Faivre, 1998 SFSA Technical \& Operating Conference.

20. T. Hays, 1998 SFSA Technical \& Operating Conference.

21. P. Scarber, Jr., 2001 SFSA Technical \& Operating Conference.

22. Ramalingam, S., "Stoichiometry of TiC and its significance to the performance of Hard Metal Compacts", Material Science and Engineering, Vol 29, 1977, p. 123-129.

23. Murshed,M., and Bates,C. E. "Effects of Foundry Processing on the Machinability of Cast Steel", Proceedings, SFSA Technical and Operating Conference, November 4-6, 1999. 


\section{USE OF THIS REPORT AND INFORMATION CONTAINED THEREIN}

Publicity

This report and the information contained therein is the property of the individual or organization named on the face hereof and may be freely distributed in its present form. However, the University of Alabama at Birmingham (UAB) hereby reminds sponsor that no advertising or publicity matter, having or containing any reference to the University of Alabama at Birmingham, shall be made use of by anyone, unless and until such matter shall have first been submitted to and received the approval in writing of UAB. (UAB does not usually approve any type of endorsement advertising.)

Limitation of Liability

The faculty and staff of UAB associated with this project have used their professional experience and best professional efforts in performing this work. However, UAB does not represent, warrant or guarantee that its research results, or product produced therefrom, are merchantable or satisfactory for any particular purpose, and there are no warranties, express or implied, to such effect. Acceptance, reliance on, or use of such results shall be at the sole risk of sponsor. In connection with this work, UAB shall in no event be responsible or liable in contract or in tort for any special, indirect, incidental or consequential damages, such as, but not limited to, loss of product, profits or revenues, damage or loss from operation or nonoperation of plant, or claims of customers of Sponsor.

To: $\quad$ Department of Energy

Idaho Operations

850 Energy Drive

Idaho Falls, ID

Date: $\quad$ February 21, 2003

UAB Account No. 527986; 537986; 537987

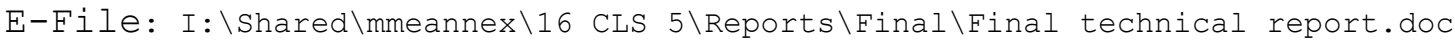

